\title{
Direct Numerical Simulation of a Turbulent Boundary Layer with Passive Scalar Transport by Qiang Li
}

April 2007

Master of Science Thesis Royal Institute of Technology

Department of Mechanics SE-100 44 Stockholm, Sweden 
Akademisk avhandling som med tillstånd av Kungliga Tekniska Högskolan i Stockholm framlägges till offentlig granskning för avläggande av civilingenjörsexamen fredagen den 21:e April $2007 \mathrm{kl} 13.30$ på Institutionen för Mekanik, Huvudbyggnaden, Kungliga Tekniska Högskolan, Osquars Backe 18, Stockholm.

(C) Qiang Li 2007

Universitetsservice US-AB, Stockholm 2007 


\title{
Abstract
}

\author{
Direct Numerical Simulation of a Turbulent \\ Boundary Layer with Passive Scalar Transport \\ Qiang Li \\ Department of Mechanics, Royal Institute of Technology \\ SE-100 44 Stockholm, Sweden
}

A direct numerical simulation (DNS) of a spatially developing turbulent boundary layer with passive scalars over a flat plate under zero pressure gradient (ZPG) is carried out. The Navier-Stokes equations are solved by employing a spectral method with $1024 \times 289 \times 128$ grid points in the streamwise, wall-normal and spanwise directions, respectively. The Reynolds number based on the free-stream velocity and inlet displacement thickness is 450 and the molecular Prandtl numbers are ranging from 0.2 to 2 , while both isothermal and isoflux wall boundary conditions are considered. An extensive number of turbulence statistics for both flow and scalar fields including the mean statistical quantities (e.g. skin friction coefficient, Stanton number, turbulent Prandtl number), turbulence intensities (e.g. root-meansquare (RMS) fluctuations, Reynolds stresses and scalar fluxes including the corresponding budgets) and higher order quantities (e.g. skewness and flatness factors) are computed and compared to existing experimental and numerical simulations at comparable Reynolds number. Agreements as well as discrepancies are discussed while the influences of the Reynolds number, molecular Prandtl number and wall boundary conditions are also highlighted. The velocity and the scalar streaks are examined through spanwise two-point correlations, and quadrant analyses and joint probability density functions (JPDF) are employed to investigate the coherence between the velocity and the scalar fluctuations. The behaviours of the Reynolds shear stress and the wall-normal scalar flux for $\mathrm{Pr}=0.71$ are similar to each other, indicating that they might be generated by the same mechanism and a close correlation exists between the streamwise velocity and the scalar for $\operatorname{Pr}=0.71$.

Descriptors: Turbulence, Direct Numerical Simulation (DNS), Turbulent Boundary Layer, Passive Scalar, Prandtl Number, Near-Wall Behaviour. 


\section{Preface}

\section{成络生我养我的妈妈和爸爸一一张宁海女士和李明先生}

历时将近半年的毕业论文终于告已段落了。在过去的半年中, 家人和朋友的关心和支持是我 完成论文的主要动力。当然, 保证了论文的顺利进行是因为两位出色指导老师菲利普 - 施拉 特 (Philipp Schlatter) 和卢卡- 布朗特 (Luca Brandt) 对我的悉心指导。他们在湍流理论, 数值模拟以及高性能计算方面广博的学识, 严谨的治学态度使我收益匪浅。在此向两位老师 致以甞高的敬意和最诚集的感激之情! 另外我要感谢赵惠茹老师和薛党鹏老师的, 我能取得 今天的成绩是和您的教诲分不开的。

在这里我想感谢我的家人和朋友给我带来的欢乐和鼓励。感谢你: 辛光, 李桓, 周帅, 关炜, 张思思, 王霏, 以及李强, 荆竟, 郑科, 沈若冰, 张通, 孔杰, 门少东, 王深石还有康㑊, 宋霏, 李桢。

感谢韩国 KBS, MBS, SBS 电视台在过去的十年中制作的无数优良的电视剧陪我度过的欢 乐时光, 希望在今后的日子里有更多的电视作品。 


\section{Contents}

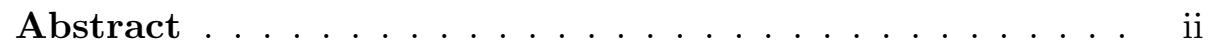

Preface ...................... iii

1 Introduction 1

2 Theoretical Formulation $\quad 6$

2.1 Governing Equations . . . . . . . . . . . . . . . 6

2.1.1 Velocity field . . . . . . . . . . . . . 6

2.1 .2 Scalar field . . . . . . . . . . . . . . . . 9

2.2 Boundary Layer Equations and Scalings . . . . . . . . . . . . 11

2.2.1 Boundary layer equations . . . . . . . . . . . 11

2.2 .2 Boundary layer scalings . . . . . . . . . . . 12

3 Direct Numerical Simulation $\quad 18$

3.1 Non-dimensional Equations . . . . . . . . . . . . . . . 18

3.2 Boundary Conditions . . . . . . . . . . . . . . . . . . . . 19

3.2.1 Boundary conditions for flow field . . . . . . . . . 19

3.2.2 Boundary conditions for scalar field . . . . . . . . 20

3.3 Computational Domain . . . . . . . . . . . . . . 20

3.4 Numerical Method . . . . . . . . . . . . . . . . . 22

3.4 .1 Numerical scheme . . . . . . . . . . . . . . 22

3.4 .2 Discretization . . . . . . . . . . . . . . 22

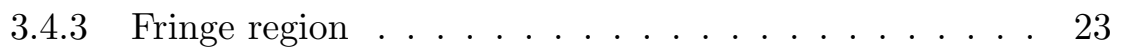

3.5 Base Flow . . . . . . . . . . . . . . . . . . . . . . . . 25

4 Averaged Results $\quad 27$

4.1 Numerical Parameters . . . . . . . . . . . . . . . . . . . 27

4.2 Hydrodynamic Results . . . . . . . . . . . . . . . . . . 28

4.2.1 Mean flow results . . . . . . . . . . . . 29

4.2 .2 Turbulent statistics . . . . . . . . . . . . . 33

4.2 .3 Vorticity fluctuations . . . . . . . . . . . . . 34

4.2.4 Reynolds stress budget . . . . . . . . . . . . . . . 36

4.2.5 Reynolds stress anisotropy . . . . . . . . . . . . . . 40

4.2 .6 Higher order statistics . . . . . . . . . . . . . 41 
4.2.7 Turbulent structure of the flow fields . . . . . . . . . . 44

4.2.8 Probability density functions . . . . . . . . . . . . . 47

4.2 .9 Quadrant analysis . . . . . . . . . . . . 47

4.3 Scalar Transport Results . . . . . . . . . . . . . . . . . . 54

4.3.1 Reynolds' analogy ..................... 54

4.3 .2 Mean scalar results . . . . . . . . . . . . . . . 55

4.3 .3 Turbulent statistics . . . . . . . . . . . . . . . . . . . 62

4.3.4 Scalar flux budget . . . . . . . . . . . . . . 67

4.3.5 Higher order statistics . . . . . . . . . . . . 71

4.3.6 Turbulent structure of the scalar fields . . . . . . . 76

4.3.7 Probability density functions . . . . . . . . . . . 77

4.3.8 Quadrant analysis ............... . . 79

4.4 Flow Field VS. Scalar Field . . . . . . . . . . . . . . . . 85

4.4.1 PDF of the Reynolds stress and scalar flux . . . . . 85

4.4 .2 Joint PDF . . . . . . . . . . . . . . . . . 88

4.4 .3 Instantaneous fields . . . . . . . . . . . . . . 89

5 Conclusion and Outlook $\quad 91$

5.1 Conclusion ....................... . . 91

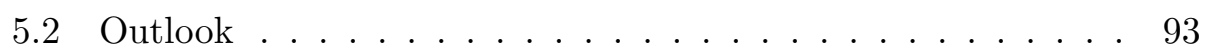

Acknowledgement . . . . . . . . . . . . . . . . 94

Bibliography . . . . . . . . . . . . . . . . . 94 


\section{List of Figures}

2.1 Mean streamwise velocity profile. . . . . . . . . . . . 17

3.1 A sketch of the fringe function $\lambda(x) \ldots \ldots \ldots . \ldots 24$

3.2 A sketch of the computational box with fringe region. . . . . 25

$4.1 R e_{\delta^{*}}$ and $R e_{\theta}$ versus $R e_{x} \ldots \ldots \ldots \ldots$. . . . . . . . 29

4.2 Mean streamwise velocity $\langle u\rangle$ profile. . . . . . . . . . . . 29

4.3 Comparison of $\langle u\rangle$ with other DNS data. . . . . . . . . . . . 30

4.4 Variation of the shape factor with the downstream positions. 31

$4.5 \delta_{99}, \delta^{*}$ and $\delta_{\theta}$ versus downstream positions. . . . . . . . . 31

4.6 Variation of skin friction coefficient $c_{f}$ with $R e_{\theta}$. . . . . . . . 32

4.7 Variation of $U_{\infty}$ and $\beta$ with downstream positions. . . . . . . 33

4.8 RMS of turbulent intensities in inner scaling. . . . . . . . . 34

4.9 RMS of turbulent intensities in outer scaling. . . . . . . . . . 35

4.10 RMS of the vorticity fluctuations at $R e_{\theta}=830 \ldots \ldots$. . . . 36

4.11 Variation of the RMS of the vorticity fluctuations with $R e_{\theta}$. . 36

4.12 Reynolds stress budgets at $R e_{\theta}=830 \ldots \ldots$. . . . . . . 37

4.13 Budget for the turbulent kinetic energy $k \ldots \ldots \ldots . \ldots 38$

4.14 Comparison of the Reynolds stress budgets at different $R e_{\theta}$. . 39

4.15 Variation of the pressure strain with wall-normal distance. . . 40

$4.16 \mathrm{AIM}$ at $R e_{\theta}=830$ versus wall-normal positions. . . . . . . . 41

4.17 Skewness factors of the velocities and pressure at $R e_{\theta}=830 . \quad 42$

4.18 Flatness factors of the velocities and pressure at $R e_{\theta}=830$. . 43

4.19 Skewness and flatness factors of the velocities and pressure at $R e_{\theta}=830 \ldots \ldots \ldots \ldots \ldots \ldots \ldots$

4.20 Spanwise two-point correlation coefficients of the velocity and pressure fluctuations at $y^{+}=4.9$ with $R e_{\theta}=830 \ldots \ldots$. . . 45

4.21 Variation of $R_{u u}$ with $R e_{\theta}$. . . . . . . . . . . . . . . . 45

4.22 Variation of the mean spanwise steak spacings with wallnormal positions at $R e_{\theta}=830 \ldots \ldots$. . . . . . . . 46

4.23 Comparison of the spanwise two-point correlation coefficients of the velocity and pressure fluctuations with other DNS data. 46

4.24 PDF of the streamwise velocity fluctuation $u^{\prime}$ at $R e_{\theta}=830$. . 48

4.25 PDF of the wall-normal velocity fluctuation $v^{\prime}$ at $R e_{\theta}=830 . \quad 49$ 
4.26 PDF of the spanwise velocity fluctuation $w^{\prime}$ at $R e_{\theta}=830 . .50$

$4.27 \mathrm{PDF}$ of the pressure fluctuation $p^{\prime}$ at $R e_{\theta}=830$. . . . . . . 51

4.28 Fractional contribution to Reynolds shear stress $\left\langle u^{\prime} v^{\prime}\right\rangle$. . . . 53

4.29 Fractional contribution to $\left\langle u^{\prime} v^{\prime}\right\rangle$ as a function of threshold $H$. 53

4.30 Mean scalar $\langle\theta\rangle$ profiles. . . . . . . . . . . . . . 56

4.31 Comparison of $\langle\theta\rangle$ with other data. . . . . . . . . . . 57

4.32 Variation of Stanton number $S t$ with $R e_{\theta} \ldots \ldots \ldots$. . . . . 58

4.33 Near wall behaviour of the turbulent Prandtl number $\operatorname{Pr}_{t}$ at $R e_{\theta}=830 \ldots \ldots \ldots \ldots \ldots \ldots$

4.34 Turbulent Prandtl number $P r_{t}$ versus the wall-normal distance at $R e_{\theta}=830 . \ldots \ldots \ldots \ldots$. . . . . . . . 60

4.35 Comparison of $\mathrm{Pr}_{t}$ with other DNS data. . . . . . . . . . . 61

$4.36 \delta_{1}^{\theta}$ and $\delta_{2}^{\theta}$ versus downstream positions. . . . . . . . . . 62

$4.37 \delta_{99}^{\theta}$ versus downstream positions. . . . . . . . . . . 63

4.38 RMS of the scalar fluctuations at different downstream positions. . . . . . . . . . . . . . . 64

4.39 Variation of the RMS at the wall with downstream positions. 65

4.40 Comparison of the RMS of the scalar fluctuations between different boundary conditions. . . . . . . . . . . . 65

4.41 Comparison of the RMS value of $\theta_{2}^{\prime}$ with other DNS data. . . 66

4.42 Variation of the scalar fluxes for isothermal boundary condition with $R e_{\theta} \ldots \ldots \ldots \ldots$. . . . . . . . . . . 67

4.43 Variation of the scalar fluxes for isoflux boundary condition with $R e_{\theta} . \ldots \ldots \ldots \ldots$. . . . . . . . . . . 68

4.44 Budgets for the fluctuating scalar variance $k_{\theta}$ at $R e_{\theta}=830$. $\quad 69$

4.45 Comparison of the budget of $k_{\theta_{2}}$ with other DNS data. . . . 70

4.46 Budgets of the scalar flux fluctuations at $R e_{\theta}=830$ for isothermal boundary condition. . . . . . . . . . . 71

4.47 Budgets of the scalar flux fluctuations at $R e_{\theta}=830$ for isoflux boundary condition. . . . . . . . . . . . 72

4.48 Comparison of the budgets of the scalar fluxes with other DNS data. . . . . . . . . . . . . . . . . . 72

4.49 Variation of the pressure scalar-gradient versus the wall-normal distances at $R e_{\theta}=830 . \ldots \ldots \ldots \ldots$. . . . . . . . 73

4.50 Variation of the skewness and flatness factors for isothermal boundary condition with wall-normal positions. . . . . . . 74

4.51 Variation of the skewness and flatness factors for isoflux boundary condition with wall-normal positions. . . . . . . 75

4.52 Comparison of skewness and flatness factors of $\theta_{2}$ with other DNS data. . . . . . . . . . . . . . . . . . 75

4.53 Spanwise two-point correlation coefficients of the scalar fluctuations at $y^{+}=9.8 \ldots \ldots \ldots \ldots \ldots 76$

4.54 Spanwise two-point correlation coefficients of the scalar fluctuations with $R e_{\theta} \ldots \ldots \ldots \ldots$. . . . . . . . . 77 
4.55 Variation of the mean spanwise scalar steaks spacing with wall-normal positions at $R e_{\theta}=830 . \ldots \ldots \ldots 78$

4.56 PDF of $\theta_{1}^{\prime}$ with $\operatorname{Pr}=0.2$ and isothermal wall and $R e_{\theta}=830.79$

4.57 PDF of $\theta_{2}^{\prime}$ with $\operatorname{Pr}=0.71$ and isothermal wall and $R e_{\theta}=830.80$

4.58 PDF of $\theta_{3}^{\prime}$ with $\operatorname{Pr}=0.71$ and isoflux wall and $R e_{\theta}=830$. . 81

4.59 PDF of $\theta_{4}^{\prime}$ with $\operatorname{Pr}=2$ and isothermal wall and $R e_{\theta}=830 . \quad . \quad 82$

4.60 PDF of $\theta_{5}^{\prime}$ with $\operatorname{Pr}=2$ and isoflux wall and $R e_{\theta}=830$. . . 83

4.61 Comparison of the PDF of $\theta_{2}^{\prime}$ with other DNS data. . . . . . 84

4.62 Fractional contribution to the wall-normal scalar flux $\left\langle v^{\prime} \theta^{\prime}\right\rangle$. . 84

4.63 Fractional contribution to $\left\langle v^{\prime} \theta^{\prime}\right\rangle$ as a function of threshold $H$. 86

4.64 PDF of $u^{\prime} v^{\prime}$ and $v^{\prime} \theta^{\prime}$ at $y^{+}=29.3$ and $R e_{\theta}=830 . \ldots . . .87$

$4.65 \mathrm{JPDF}$ of $\left(u^{\prime}, \theta^{\prime}\right)$ at $y^{+} \approx 5$ and $R e_{\theta}=830 \ldots \ldots . . . . .88$

4.66 JPDF of $\left(u^{\prime}, v^{\prime}\right)$ and $\left(v^{\prime}, \theta^{\prime}\right)$ at $y^{+} \approx 5$ and $R e_{\theta}=830 . \ldots . \quad 89$

4.67 Instantaneous flow and scalar fields at $y^{+} \approx 7 . \ldots . . . .99$ 


\section{List of Tables}

4.1 Parameters for the direct numerical simulation. . . . . . . . . 28

4.2 List of various Reynolds numbers. . . . . . . . . . . . 28

4.3 Classification of the fluid motion in $(u, v)$-plane. . . . . . . . 52

4.4 List of the different scalars. . . . . . . . . . . . . . 54 
Somebody did a golden deed;

Somebody proved a friend in need;

Somebody sang a beautiful song;

Somebody smiled the whole day long;

Somebody thought, "Tis sweet to live";

Somebody said, "I'm glad to give";

Somebody fought a valiant fight;

Somebody lived to shield the right;

Was the "somebody" you?

— somebody

Fröken Lise vid fjorton år

Snörd och rak och sedig går,

Alla dar moraler får

Af en fransk bedagad hexa,

Dansar, spelar, får beröm,

Skådar englar i hvar dröm,

Tecknar blommor på sin söm,

Medan egna blomster växa.

Unga Lindor, dödligt kär,

Snart den skönas hand begär,

Häftig, varm och trägen är,

Lofvar dyrt att evigt brinna.

Ringar, nipper, bref och bud,

Klagan, tjusning, brutna ljud,

Offer till de giftas gud -

$\mathrm{Nu}$ är fröken Lise grefvinna. 


\section{Chapter 1}

\section{Introduction}

Turbulence is a universal phenomenon. Most flows in nature and engineering applications are turbulent, i.e. the flow field fluctuates rapidly in both time and space. Examples of such flows include billowing of clouds in the sky, oceanic currents, the flow over the wing of an aircraft or the flow over a gas turbine blade. Some flows in everyday life are deliberately made to be turbulent, e.g. coffee stirring, tea pouring and cocktail shaking. Such inherent random and complex characteristics make turbulence to be an intellectual challenge for a great number of scientists and engineers and will continue to pose this challenge in the future. Consequently, a huge amount of theoretical and experimental work was done during the last two centuries to shed some light on this mysterious field.

The first example of a visualisation of a flow developing from laminar into turbulent motion is the famous experiment carried out by Osborne Reynolds in 1883. He studied the flow of water in a glass tube using ink as a dye. For low flow rates a steady dye stream was observed to follow a straight path through the tube. As the flow rate was increased, at some point, "the colour band would all at once mix up with the surrounding water, and fill the rest of the tube with a mass of coloured water". In order to quantify these experimental results, he introduced a non-dimensional number $R e=$ $\frac{U L}{\nu}$, now known as the Reynolds number, in his classic paper published later (Here $U$ denotes the velocity scale, $L$ the length scale and $\nu$ is the kinematic viscosity). The flow will become turbulent if the Reynolds number exceeds a critical value. The Reynolds number can be interpreted as a measure of the ratio between the inertial and viscous forces acting on a fluid particle and subsequently proved to be the parameter that determines the dynamic similarity of the viscous flows. It is by far one of the most important dimensionless numbers in fluid mechanics.

Another typical example demonstrating the nature of a turbulent flow is the meandering of the smoke from a cigarette, see e.g. Libby (1996). The smoke first moves straight upward and suddenly changes to a turbulent sta- 
tus. This experiment not only provides the visualisation of the turbulence but also the spreading of a passive scalar in a turbulent flow. A passive scalar is a diffusive contaminant in a fluid flow that, due to its low concentration, has no influence on the fluid motion but however is influenced by the fluid motion. Typical passive scalars are weakly heated flow, i.e. small amounts of heat, or pollutants in atmospheric or ocean flows. An understanding and prediction of the passive scalar behaviour in a turbulent flow is crucial since the turbulent momentum and scalar transport play a key role in many engineering applications and will be of growing importance in global environmental problems (Kasagi and Iida, 1999).

When a fluid flows above a solid body, the fluid elements in a thin layer near the body surface will be retarded due to the effect of friction within the fluid while the other elements outside this thin layer are not affected. This thin layer is usually called the boundary layer. The concept of the boundary layer was first proposed by Ludwig Prandtl in 1905. He hypothesised that the viscous effects were negligible everywhere except in a thin layer close to the solid boundary of the body where the no-slip condition had to be fulfilled. In the boundary layer many interesting parameters of the aerodynamic property of the body are determined. Thus it is of great engineering significance in most applications.

Although the phenomena of turbulent flows vary from one to the other, the governing equations describing the motion of the Newtonian fluids are always the same. These equations are called Navier-Stokes (N-S) equations, named after Claude-Louis Navier and George Gabriel Stokes who first formulated them in 19th century. Combined with the continuity equation (conservation of mass) and the scalar transport equation which governs the evolution of the scalar field, a system of five equations consisting of five variables (three velocity components, pressure and scalar concentration) is established. However, the N-S equations are non-linear and time dependent partial differential equations, therefore no analytical solutions exists except for some very simple flow cases. So one has to calculate the solutions of the N-S equations numerically with the aid of supercomputers.

Due to the rapid progress in high performance computers, the direct numerical simulation (DNS) became an important tool for turbulence research in the past couple of decades (Moin and Mahesh, 1998). A review of the turbulent heat transfer at the same time is made by Kasagi and Iida (1999). DNS provides three dimensional, time-dependent numerical solutions to the $\mathrm{N}-\mathrm{S}$ equations and the scalar transport equation. These equations are supposed to be solved as accurately as possible without employing any turbulence models, hence a DNS must be performed on a fine numerical grid in order to capture all the scales arising in the turbulent flow. It should be noted that the Reynolds number in typical engineering application flows are usually quite high and an increase of the Reynolds number gives rise to the excitation of smaller and smaller turbulent scales, e.g. see Pope (2000). A 
simple scaling argument leads to the conclusion that the computational time for a DNS, in which one resolves the Kolmogorov viscous scales, i.e. all the relevant flow structures, is roughly proportional to $\operatorname{Re}^{3}$ (Moin and Mahesh, 1998). Therefore an enormous challenge for the supercomputer when performing DNS is the wide range of the scales which makes the simulation large and time consuming. Despite the demanding nature, DNS has proven to be a very useful and efficient tool for the turbulence research (Moin and Mahesh, 1998; Kasagi and Iida, 1999).

For the DNS of the turbulent scalar transfer, not only the Reynolds number $(R e)$ but also the molecular Prandtl number $(P r)$ has to be considered. The molecular Prandtl number is a dimensionless number which is a measure of the ratio between viscous diffusion and scalar diffusion. Typical values for $\operatorname{Pr}$ are 0.71 for air and many other gases, around 7 for water, between 100 and 40,000 for engine oil and around 0.025 for mercury (White, 2006). The first such direct numerical simulations were performed by Rogers et al. (1986) in a homogeneous shear flow and by Kim and Moin (1989) in a channel flow of $\operatorname{Pr}=0.1,0.71$ and 2.0 with $R e_{\tau}=180$ where $R e_{\tau}$ is the Reynolds number based on the friction velocity $u_{\tau}$ and the channel half width $h$. Later, Lyons and Hanratty (1991) made a similar DNS of $u_{\tau}=150$ and $\operatorname{Pr}=1$. Kasagi et al. (1992) and Kasagi and Ohtsubo (1993) performed DNS at $R e_{\tau}=150$ and $P r=0.71$ and 0.025. Wikström (1998) made the DNS with a higher Reynolds number of $R e_{\tau}=265$ while $\operatorname{Pr}=0.71$. Abe et al. (2004) made a DNS with $R e_{\tau}$ up to 1024 and $P r=0.025$ and 0.71 . Chung and Sung (2003) did a DNS of a turbulent concentric annular pipe flow at $R e_{D_{h}}=8900$ which is based on the bulk mean velocity $U_{m}$ and the hydraulic diameter $D_{h}$ and $P r=0.71$. All these simulations are done, however, with a $\operatorname{Pr}$ no more than two. This is because the smallest scales in the scalar fluctuation decrease with the increase of $\mathrm{Pr}$. Therefore the DNS becomes an even more difficult task when the Prandtl number is high. The ratio of the largest to the smallest scale is approximately proportional to $\operatorname{Re}^{\frac{3}{4}} \operatorname{Pr}^{\frac{1}{2}}$ at very high $\operatorname{Pr}$ (Batchelor, 1959; Tennekes and Lumley, 1972). With the help of larger parallel computers, Kawamura et al. (1998) performed the DNS in periodic channel flow at $R e_{\tau}=180$ but for a wider range of $\operatorname{Pr}$ from 0.025 to 5.0. Na and Hanratty (2000) ran a channel flow DNS reaching $\operatorname{Pr}$ of 10 with $R e_{\tau}=150$.

So far the DNS of the turbulent scalar transfer, e.g. heat transfer, is mainly focused on fully developed turbulent channel flow because of its simple geometry and the nature to reveal the mechanism of convective heat transfer between fluid and a solid wall. Only few DNS were done for a thermal turbulent boundary layer even its geometry is not too complex. As reported in Kong et al. (2000), D. M. Bell and J. H. Ferziger were the first to run a direct numerical simulation of a thermal boundary layer. Later Kong et al. (2000) did a DNS of thermal boundary layer at $R e_{\theta}=300$ and the $\operatorname{Pr}=0.71$ where $\operatorname{Re}_{\theta}$ is based on the free-stream velocity $U_{\infty}$ and the 
inlet momentum thickness $\theta$.

Even though the use of numerical tools for studying scalar transfer steadily increased in the last decades, experimental measurements can not be replaced by numerical simulation. For many flow cases, numerical results do not give reliable predictions of scalar transfer because of uncertainty in turbulence models or flow conditions. Under such a circumstance, experimental measurements are needed to determine the performance of a thermal system. On the other hand, experimental results are required to verify the numerical methods and turbulence models.

The understanding of the spreading of a passive scalar in turbulent flows was initially gained through wind tunnel experiments. The early studies of heat transfer were done by Corrsin (1952), Warhaft and Lumley (1978), Tavoularis and Corrsin (1981) and Sirivat and Warhaft (1983) in gridgenerated turbulence and homogeneous turbulence. For channel, pipe and boundary layer flows, the experiments before 1970 are reviewed in the paper by Kader and Yaglom (1972). During the last twenty years, Hishida and Nagano (1979) and Nagano and Tagawa (1995) did experiments in fully developed pipe flow to investigate the transport mechanism in turbulence to correlate the transfer processes of momentum and scalar with coherent motions. Nagano and Tagawa (1988) measured various types of moments of velocity and scalar fluctuations in a fully developed pipe flow. Zhu and Antonia (1993) measured the temperature dissipation in a fully developed turbulent channel flow. Later, Mosyak et al. (2001) and Hetsroni et al. (2001) carried out experiments to study the wall temperature fluctuations under different wall boundary conditions, and also the thermal coherent structure in a fully developed channel flow. For the turbulent boundary layer flows, Hoffmann and Perry (1979) examined the similarity between the Reynolds shear stress $\left\langle u^{\prime} v^{\prime}\right\rangle$ and scalar flux $\left\langle v^{\prime} \theta^{\prime}\right\rangle$. Iritani et al. (1985) examined the relation between the coherent structures and the temperature fluctuations near the wall. Krishnamoorthy and Antonia (1987) and Antonia et al. (1988) did experiments to investigate the temperature dissipation and the correlation between the longitudinal velocity fluctuation and temperature fluctuation in the near wall region. Anselmet et al. (1994) investigated the statistical relationship between a passive scalar and its dissipation. Benaissa et al. (1999) studied of the conditional correlation between a passive scalar and its dissipation.

This thesis is a study of passive scalar transport in a turbulent boundary layer spatially developing over a flat plate with zero pressure gradient (ZPG). The investigation is performed using direct numerical simulation (DNS). The $R e_{\delta_{0}^{*}}$ based on the free-stream velocity $U_{\infty}$ and the inlet displacement thickness $\delta_{0}^{*}$ is 450 and $\operatorname{Pr}$ are chosen as $0.2,0.71$ and 2.0. Isothermal and isoflux wall boundary conditions are employed for comparison. The numerical code used for the present DNS was developed by Lundbladh et al. (1999) at the Royal Institute of Technology (KTH). A spectral method is 
adopted in this code which is more accurate than the finite element or finite difference method. Therefore less numerical errors are expected and this is very important for a numerical simulation. The goal of this thesis is to extend our knowledge about the scalar turbulent boundary layer flows to a wider range of both Reynolds number and Prandtl numbers and also to generate a data base for the research community. 


\section{Chapter 2}

\section{Theoretical Formulation}

\subsection{Governing Equations}

\subsubsection{Velocity field}

The governing equations for an incompressible (laminar and turbulent) flow of a viscous fluid are the Navier-Stokes equation and the continuity equation, here written in tensor notation

$$
\begin{aligned}
\frac{D u_{i}}{D t} \equiv \frac{\partial u_{i}}{\partial t}+u_{j} \frac{\partial u_{i}}{\partial x_{j}} & =-\frac{1}{\rho} \frac{\partial p}{\partial x_{i}}+\nu \frac{\partial^{2} u_{i}}{\partial x_{j} \partial x_{j}}, \\
\frac{\partial u_{i}}{\partial x_{i}} & =0
\end{aligned}
$$

where the operator $\frac{D}{D t}$ is the material derivative which denotes the rate of change in a coordinate system following the local velocity field. $\left(x_{1}, x_{2}, x_{3}\right)$ $=(x, y, z)$ are the Cartesian coordinates in the streamwise, wall-normal and spanwise direction, respectively, $\left(u_{1}, u_{2}, u_{3}\right)=(u, v, w)$ are the corresponding instantaneous velocity fields, $p$ is the total pressure while $\rho$ and $\nu$ are the density and kinematic viscosity of the fluid. The summation convention is implied over repeated indices. The streamwise and spanwise directions will be alternatively termed as the horizontal directions.

In many applications, it is the mean flow rather than the rapid fluctuations that is interesting and important. Naturally the Reynolds decomposition of the flow field variables into a mean and a fluctuating quantity is introduced by

$$
\begin{gathered}
u_{i}=\left\langle u_{i}\right\rangle+u_{i}^{\prime}, \\
p=\langle p\rangle+p^{\prime},
\end{gathered}
$$

where the angular brackets denote the mean part and a prime denotes the fluctuating part. In a statistically stationary turbulent flow, the mean value of a flow quantity can be taken as the time average or as an average in the 
homogeneous directions. Generally the mean of a quantity is the ensemble average over an infinite number of realizations. In the present case the mean of a quantity $f(x, y, z, t)$ is defined by

$$
\langle f\rangle(x, y)=\lim _{T \rightarrow \infty} \frac{1}{T} \int_{0}^{\infty} f(x, y, z, t) \mathrm{d} t,
$$

where the $z$ dependence disappears since it is a homogeneous direction.

By inserting the Reynolds decompositions into the equations (2.1), (2.2) and taking the average, we obtain the Reynolds averaged Navier-Stokes (RANS) and the continuity equations

$$
\begin{gathered}
\frac{\partial\left\langle u_{i}\right\rangle}{\partial t}+\left\langle u_{j}\right\rangle \frac{\partial\left\langle u_{i}\right\rangle}{\partial x_{j}}=-\frac{1}{\rho} \frac{\partial\langle p\rangle}{\partial x_{i}}+\frac{\partial}{\partial x_{j}}\left(\nu \frac{\partial\left\langle u_{i}\right\rangle}{\partial x_{j}}-\left\langle u_{i}^{\prime} u_{j}^{\prime}\right\rangle\right), \\
\frac{\partial\left\langle u_{i}\right\rangle}{\partial x_{i}}=0 .
\end{gathered}
$$

The quantity $\left\langle u_{i}^{\prime} u_{j}^{\prime}\right\rangle$ in equation (2.6) is the Reynolds stress tensor which originates from the non-linear convection term in equation (2.1). One can never solve the equation (2.6) by introducing transport equation of the Reynolds stress tensor since higher order moments of the turbulent field will be involved in the newly introduced transport equation. This is the so-called closure problem.

The transport equation for the Reynolds stress tensor is obtained by multiplying equation (2.1) (after subtracting the mean equation (2.6)) with $u_{j}$, adding the corresponding equation with switched indices $i, j$ and taking the average. The resulting equation reads

$$
\begin{aligned}
\frac{\partial\left\langle u_{i}^{\prime} u_{j}^{\prime}\right\rangle}{\partial t} & +\left\langle u_{l}\right\rangle \frac{\partial\left\langle u_{i}^{\prime} u_{j}^{\prime}\right\rangle}{\partial x_{l}}=-\left(\left\langle u_{j}^{\prime} u_{l}^{\prime}\right\rangle \frac{\partial\left\langle u_{i}\right\rangle}{\partial x_{l}}+\left\langle u_{i}^{\prime} u_{l}^{\prime}\right\rangle \frac{\partial\left\langle u_{j}\right\rangle}{\partial x_{l}}\right) \\
& +\frac{1}{\rho}\left(\left\langle p^{\prime} \frac{\partial u_{i}^{\prime}}{\partial x_{j}}\right\rangle+\left\langle p^{\prime} \frac{\partial u_{j}^{\prime}}{\partial x_{i}}\right\rangle\right)-\frac{1}{\rho} \frac{\partial}{\partial x_{l}}\left(\left\langle u_{i}^{\prime} p\right\rangle \delta_{j l}+\left\langle u_{j}^{\prime} p\right\rangle \delta_{i l}\right) \\
& -\frac{\partial\left\langle u_{i}^{\prime} u_{j}^{\prime} u_{l}^{\prime}\right\rangle}{\partial x_{l}}+\nu \frac{\partial^{2}\left\langle u_{i}^{\prime} u_{j}^{\prime}\right\rangle}{\partial x_{l} \partial x_{l}}-2 \nu\left\langle\frac{\partial u_{i}^{\prime}}{\partial x_{l}} \frac{\partial u_{j}^{\prime}}{\partial x_{l}}\right\rangle
\end{aligned}
$$

where $\delta_{i j}$ is Kronecker delta, defined by

$$
\delta_{i j}=\left\{\begin{array}{ll}
1 & \text { if } i=j \\
0 & \text { if } i \neq j
\end{array} .\right.
$$

The different terms in equation (2.8) are denoted as

$$
\begin{aligned}
& R_{i j} \equiv\left\langle u_{i}^{\prime} u_{j}^{\prime}\right\rangle \\
& \mathcal{P}_{i j} \equiv-\left\langle u_{j}^{\prime} u_{l}^{\prime}\right\rangle \frac{\partial\left\langle u_{i}\right\rangle}{\partial x_{l}}-\left\langle u_{i}^{\prime} u_{l}^{\prime}\right\rangle \frac{\partial\left\langle u_{j}\right\rangle}{\partial x_{l}}
\end{aligned}
$$




$$
\begin{aligned}
\Pi_{i j} & \equiv \frac{1}{\rho}\left(\left\langle p^{\prime} \frac{\partial u_{i}^{\prime}}{\partial x_{j}}\right\rangle+\left\langle p^{\prime} \frac{\partial u_{j}^{\prime}}{\partial x_{i}}\right\rangle\right), \\
G_{i j} & \equiv-\frac{1}{\rho} \frac{\partial}{\partial x_{l}}\left(\left\langle u_{i}^{\prime} p\right\rangle \delta_{j l}+\left\langle u_{j}^{\prime} p\right\rangle \delta_{i l}\right), \\
T_{i j} & \equiv-\frac{\partial\left\langle u_{i}^{\prime} u_{j}^{\prime} u_{l}^{\prime}\right\rangle}{\partial x_{l}}, \\
D_{i j} & \equiv \nu \frac{\partial^{2}\left\langle u_{i}^{\prime} u_{j}^{\prime}\right\rangle}{\partial x_{l} \partial x_{l}}, \\
\varepsilon_{i j} & \equiv 2 \nu\left\langle\frac{\partial u_{i}^{\prime}}{\partial x_{l}} \frac{\partial u_{j}^{\prime}}{\partial x_{l}}\right\rangle,
\end{aligned}
$$

where $R_{i j}$ is the Reynolds stress tensor, $\mathcal{P}_{i j}$ is the production term due to mean flow field gradient, whose trace $\left(\mathcal{P}_{i i}\right)$ represents twice the production of turbulent energy which describes the transfer of energy from the mean flow to the turbulent fluctuations. $\Pi_{i j}$ is the pressure-strain rate correlation tensor, which is traceless, i.e. $\Pi_{i i}=0$ and represents the inter-component redistribution of the turbulent energy between Reynolds stress terms. $G_{i j}$ is the divergence of the pressure-velocity correlation, also known as the pressure diffusion term, which represents spatial redistribution of the energy among different Reynolds stress components due to inhomogeneities in the flow field. $T_{i j}$ is the turbulent diffusion term which is the divergence of the triple correlation tensor, acting as a spatial redistribution term. $D_{i j}$ is the molecular diffusion term acting to even out the turbulent stresses by spatial redistribution, whereas $\varepsilon_{i j}$ is the viscous dissipation rate tensor acting as a destruction term of turbulent kinetic energy and stresses. The transport equation for Reynolds stress can thus be rewritten as

$$
\frac{\bar{D} R_{i j}}{\bar{D} t} \equiv \frac{\partial R_{i j}}{\partial t}+\left\langle u_{l}\right\rangle \frac{\partial R_{i j}}{\partial x_{l}}=\mathcal{P}_{i j}+\Pi_{i j}+G_{i j}+T_{i j}+D_{i j}-\varepsilon_{i j} .
$$

The transport equation for the kinetic energy $k \equiv \frac{1}{2}\left\langle u_{i}^{\prime} u_{i}^{\prime}\right\rangle$ is

$$
\frac{\partial k}{\partial t}+\left\langle u_{j}\right\rangle \frac{\partial k}{\partial x_{j}}=-\left\langle u_{i}^{\prime} u_{j}^{\prime}\right\rangle \frac{\partial\left\langle u_{i}\right\rangle}{\partial x_{j}}-\frac{1}{\rho} \frac{\partial\left\langle u_{j}^{\prime} p\right\rangle}{\partial x_{j}}-\frac{1}{2} \frac{\partial\left\langle u_{i}^{\prime} u_{i}^{\prime} u_{j}^{\prime}\right\rangle}{\partial x_{j}}+2 \nu\left\langle u_{i}^{\prime} \frac{\partial^{2} u_{i}^{\prime}}{\partial x_{j} \partial x_{j}}\right\rangle,
$$

where the last term on the right hand side, $2 \nu\left\langle u_{i}^{\prime} \frac{\partial^{2} u_{i}^{\prime}}{\partial x_{j} \partial x_{j}}\right\rangle$ can be split into a molecular diffusion term $\mathcal{D}_{K}^{p}$ and a pseudo dissipation term $\varepsilon_{K}^{p}$ or a viscous diffusion term $\mathcal{D}_{K}$ and a true dissipation term $\varepsilon_{K}$ as follows

$$
2 \nu\left\langle u_{i}^{\prime} \frac{\partial^{2} u_{i}^{\prime}}{\partial x_{j} \partial x_{j}}\right\rangle=\nu \frac{\partial^{2} k}{\partial x_{j} \partial x_{j}}-\nu\left\langle\frac{\partial u_{i}^{\prime}}{\partial x_{j}} \frac{\partial u_{i}^{\prime}}{\partial x_{j}}\right\rangle
$$

or

$$
2 \nu\left\langle u_{i}^{\prime} \frac{\partial^{2} u_{i}^{\prime}}{\partial x_{j} \partial x_{j}}\right\rangle=2 \nu \frac{\partial\left\langle u_{i}^{\prime} s_{i j}^{\prime}\right\rangle}{\partial x_{j}}-2 \nu\left\langle s_{i j}^{\prime} s_{i j}^{\prime}\right\rangle,
$$


where $s_{i j}^{\prime}$ is the fluctuating strain rate tensor defined by

$$
s_{i j}^{\prime}=\frac{1}{2}\left(\frac{\partial u_{i}^{\prime}}{\partial x_{j}}+\frac{\partial u_{j}^{\prime}}{\partial x_{i}}\right) .
$$

The transport equation (2.12) can be written as

$$
\frac{\bar{D} k}{\bar{D} t} \equiv \frac{\partial k}{\partial t}+\left\langle u_{j}\right\rangle \frac{\partial k}{\partial x_{j}}=\mathcal{P}_{k}+G_{k}+T_{k}+\mathcal{D}_{k}^{p}-\varepsilon_{k}^{p}
$$

or

$$
\frac{\bar{D} k}{\bar{D} t} \equiv \frac{\partial k}{\partial t}+\left\langle u_{j}\right\rangle \frac{\partial k}{\partial x_{j}}=\mathcal{P}_{k}+G_{k}+T_{k}+\mathcal{D}_{k}-\varepsilon_{k} .
$$

The different terms on the right hand side of the equation (2.16) and equation (2.17) are denoted as

$$
\begin{aligned}
\mathcal{P}_{k} & \equiv \frac{1}{2} \mathcal{P}_{i i}=-\left\langle u_{i}^{\prime} u_{j}^{\prime}\right\rangle \frac{\partial\left\langle u_{i}\right\rangle}{\partial x_{j}} \\
G_{k} & \equiv \frac{1}{2} G_{i i}=-\frac{1}{\rho} \frac{\partial\left\langle u_{j}^{\prime} p^{\prime}\right\rangle}{\partial x_{j}} \\
T_{k} & \equiv \frac{1}{2} T_{i i}=-\frac{1}{2} \frac{\partial\left\langle u_{i}^{\prime} u_{i}^{\prime} u_{j}^{\prime}\right\rangle}{\partial x_{j}} \\
\varepsilon_{k}^{p} & \equiv \frac{1}{2} \varepsilon_{i i}=\nu\left\langle\frac{\partial u_{i}^{\prime}}{\partial x_{j}} \frac{\partial u_{i}^{\prime}}{\partial x_{j}}\right\rangle \\
\varepsilon_{k} & \equiv 2 \nu\left\langle s_{i j}^{\prime} s_{i j}^{\prime}\right\rangle \\
\mathcal{D}_{k}^{p} & \equiv \frac{1}{2} \mathcal{D}_{i i}=\nu \frac{\partial^{2} k}{\partial x_{j} \partial x_{j}} \\
\mathcal{D}_{k} & \equiv 2 \nu \frac{\partial\left\langle u_{i}^{\prime} s_{i j}^{\prime}\right\rangle}{\partial x_{j}}
\end{aligned}
$$

where $\mathcal{P}_{k}$ is the turbulent energy production, $\varepsilon_{k}$ is the true viscous dissipation, $\varepsilon_{k}^{p}$ is the pseudo dissipation, $G_{k}$ is the pressure diffusion, $T_{k}$ is the turbulent diffusion, $\mathcal{D}_{k}$ and $\mathcal{D}_{k}^{p}$ are the viscous diffusion and the molecular diffusion.

Actually the difference between the true dissipation $\varepsilon_{k}$ and pseudo dissipation $\varepsilon_{k}^{p}$ of turbulent kinetic energy $k$ is quite small. In almost all circumstances, the distinction of these two terms is not important.

\subsubsection{Scalar field}

The governing equations for a passive scalar share many similarities with those of the velocity field for an incompressible flow of a viscous fluid. The transport equation for a passive scalar is given by

$$
\frac{\partial \theta}{\partial t}+u_{i} \frac{\partial \theta}{\partial x_{i}}=\alpha \frac{\partial^{2} \theta}{\partial x_{i} \partial x_{i}},
$$


where $\theta$ is the passive scalar, e.g. temperature or pollutant concentration, and $\alpha$ the scalar molecular diffusivity. Again, the summation convention is implied over repeated indices.

In analogy to the Reynolds decomposition of the velocity field, the instantaneous scalar may also be split into a mean and a fluctuating part

$$
\theta=\langle\theta\rangle+\theta^{\prime} .
$$

By inserting this decomposition into equation (2.25) and taking average, we obtain the averaged transport equations for the scalar

$$
\frac{\partial\langle\theta\rangle}{\partial t}+\left\langle u_{i}\right\rangle \frac{\partial\langle\theta\rangle}{\partial x_{i}}=\frac{\partial}{\partial x_{i}}\left(\alpha \frac{\partial\langle\theta\rangle}{\partial x_{i}}-\left\langle u_{i}^{\prime} \theta^{\prime}\right\rangle\right) .
$$

The scalar flux term $\left\langle u_{i}^{\prime} \theta^{\prime}\right\rangle$ in equation (2.27), due to the non-linear convection term in (2.25), leaves the equation unclosed.

The transport equation for the scalar flux is given by

$$
\begin{aligned}
\frac{\partial\left\langle u_{i}^{\prime} \theta^{\prime}\right\rangle}{\partial t} & +\left\langle u_{l}\right\rangle \frac{\partial\left\langle u_{i}^{\prime} \theta^{\prime}\right\rangle}{\partial x_{l}}=-\left(\left\langle u_{l}^{\prime} \theta^{\prime}\right\rangle \frac{\partial\left\langle u_{i}\right\rangle}{\partial x_{l}}+\left\langle u_{i}^{\prime} u_{l}^{\prime}\right\rangle \frac{\partial\langle\theta\rangle}{\partial x_{l}}\right) \\
& +\frac{1}{\rho}\left\langle p^{\prime} \frac{\partial \theta^{\prime}}{\partial x_{i}}\right\rangle-\frac{1}{\rho} \frac{\partial\left\langle p^{\prime} \theta^{\prime}\right\rangle}{\partial x_{i}}-\frac{\partial\left\langle u_{i}^{\prime} u_{l}^{\prime} \theta^{\prime}\right\rangle}{\partial x_{l}} \\
& +\frac{\partial}{\partial x_{l}}\left(\alpha\left\langle u_{i}^{\prime} \frac{\partial \theta^{\prime}}{\partial x_{l}}\right\rangle+\nu\left\langle\theta^{\prime} \frac{\partial u_{i}^{\prime}}{\partial x_{l}}\right\rangle\right)-(\nu+\alpha)\left\langle\frac{\partial u_{i}^{\prime}}{\partial x_{l}} \frac{\partial \theta^{\prime}}{\partial x_{l}}\right\rangle,
\end{aligned}
$$

which is similar to that for the Reynolds stress, i.e. equation (2.8). The different terms on the right hand side of the equation (2.28) are

$$
\begin{aligned}
\mathcal{P}_{\theta i} & \equiv-\left\langle u_{l}^{\prime} \theta^{\prime}\right\rangle \frac{\partial\left\langle u_{i}\right\rangle}{\partial x_{l}}+\left\langle u_{i}^{\prime} u_{l}^{\prime}\right\rangle \frac{\partial\langle\theta\rangle}{\partial x_{l}}, \\
\Pi_{\theta i} & \equiv \frac{1}{\rho}\left\langle p^{\prime} \frac{\partial \theta^{\prime}}{\partial x_{i}}\right\rangle, \\
G_{\theta i} & \equiv-\frac{1}{\rho} \frac{\partial\left\langle p^{\prime} \theta^{\prime}\right\rangle}{\partial x_{i}}, \\
T_{\theta i} & \equiv-\frac{\partial\left\langle u_{i}^{\prime} u_{l}^{\prime} \theta^{\prime}\right\rangle}{\partial x_{l}}, \\
\mathcal{D}_{\theta i} & \equiv \frac{\partial}{\partial x_{l}}\left(\alpha\left\langle u_{i}^{\prime} \frac{\partial \theta^{\prime}}{\partial x_{l}}\right\rangle+\nu\left\langle\theta^{\prime} \frac{\partial u_{i}^{\prime}}{\partial x_{l}}\right\rangle\right), \\
\varepsilon_{\theta i} & \equiv(\nu+\alpha)\left\langle\frac{\partial u_{i}^{\prime}}{\partial x_{l}} \frac{\partial \theta^{\prime}}{\partial x_{l}}\right\rangle,
\end{aligned}
$$

where $\mathcal{P}_{\theta i}$ is the production term due to both the mean gradients of velocity and scalar, $\Pi_{\theta i}$ is the pressure scalar-gradient correlation term, $G_{\theta i}$ is the divergence of the pressure-scalar correlation term, $T_{\theta i}$ is the turbulent diffusion term, $\mathcal{D}_{\theta i}$ is the molecular diffusion term and $\varepsilon_{\theta i}$ the dissipation term, 
respectively. Thus the transport equation of scalar flux written in symbolic form is

$$
\frac{\bar{D}\left\langle u_{i}^{\prime} \theta^{\prime}\right\rangle}{\bar{D} t}=\mathcal{P}_{\theta i}+\Pi_{\theta i}+G_{\theta i}+T_{\theta i}+\mathcal{D}_{\theta i}-\varepsilon_{\theta i} .
$$

The transport equation for half of the scalar variance, $k_{\theta} \equiv \frac{1}{2}\left\langle\theta^{\prime} \theta^{\prime}\right\rangle$, which corresponds to the turbulent kinetic energy $k$, is obtained by multiplying equation (2.25) (after subtracting the mean equation (2.27)) with the fluctuating scalar $\theta^{\prime}$ and then taking the average. This yields

$$
\frac{\partial k_{\theta}}{\partial t}+\left\langle u_{l}\right\rangle \frac{\partial k_{\theta}}{\partial x_{l}}=-\left\langle u_{l}^{\prime} \theta^{\prime}\right\rangle \frac{\partial\langle\theta\rangle}{\partial x_{l}}-\frac{1}{2} \frac{\partial\left\langle u_{l}^{\prime} \theta^{\prime} \theta^{\prime}\right\rangle}{\partial x_{l}}+\alpha \frac{\partial^{2} k_{\theta}}{\partial x_{l} \partial x_{l}}-\alpha\left\langle\frac{\partial \theta^{\prime}}{\partial x_{l}} \frac{\partial \theta^{\prime}}{\partial x_{l}}\right\rangle .
$$

The different terms on the right hand side of the (2.36) are denoted as

$$
\begin{aligned}
\mathcal{P}_{\theta} & \equiv-\left\langle u_{l}^{\prime} \theta^{\prime}\right\rangle \frac{\partial\langle\theta\rangle}{\partial x_{l}}, \\
T_{\theta} & \equiv-\frac{1}{2} \frac{\partial\left\langle u_{l}^{\prime} \theta^{\prime} \theta^{\prime}\right\rangle}{\partial x_{l}}, \\
\mathcal{D}_{\theta} & \equiv \alpha \frac{\partial^{2} k_{\theta}}{\partial x_{l} \partial x_{l}}, \\
\varepsilon_{\theta} & \equiv \alpha\left\langle\frac{\partial \theta^{\prime}}{\partial x_{l}} \frac{\partial \theta^{\prime}}{\partial x_{l}}\right\rangle,
\end{aligned}
$$

where $\mathcal{P}_{\theta}$ is the production which is due to the mean scalar gradient, $\varepsilon_{\theta}$ is the dissipation rate of $k_{\theta}, \mathcal{D}_{\theta}$ and $T_{\theta}$ are the molecular and turbulent diffusion of $k_{\theta}$. The transport equation for $k_{\theta}$ rewritten in symbolic form is

$$
\frac{\bar{D} k_{\theta}}{\bar{D} t}=\mathcal{P}_{\theta}+T_{\theta}+\mathcal{D}_{\theta}-\varepsilon_{\theta} .
$$

\subsection{Boundary Layer Equations and Scalings}

\subsubsection{Boundary layer equations}

In a statistically stationary and two-dimensional boundary layer with the standard boundary layer approximations, i.e. negligible streamwise diffusion $\left(\frac{\partial}{\partial x} \ll \frac{\partial}{\partial y}\right)$ and constant pressure through the boundary layer $(\langle p\rangle=\langle p\rangle(x))$, the mean flow equations $(2.6),(2.27)$ and (2.7) reduce to

$$
\begin{gathered}
\langle u\rangle \frac{\partial\langle u\rangle}{\partial x}+\langle v\rangle \frac{\partial\langle u\rangle}{\partial y}=-\frac{1}{\rho} \frac{d\langle p\rangle}{d x}+\frac{\partial}{\partial y}\left(\nu \frac{\partial\langle u\rangle}{\partial y}-\left\langle u^{\prime} v^{\prime}\right\rangle\right) \\
\langle u\rangle \frac{\partial\langle\theta\rangle}{\partial x}+\langle v\rangle \frac{\partial\langle\theta\rangle}{\partial y}=\frac{\partial}{\partial y}\left(\alpha \frac{\partial\langle\theta\rangle}{\partial y}-\left\langle v^{\prime} \theta^{\prime}\right\rangle\right) \\
\frac{\partial\langle u\rangle}{\partial x}+\frac{\partial\langle v\rangle}{\partial y}=0
\end{gathered}
$$


Note that in equation (2.42), we use $\frac{d\langle p\rangle}{d x}$ instead of $\frac{\partial\langle p\rangle}{\partial x}$. That is the result of having taken into account the boundary layer approximation for the pressure, i.e. $\langle p\rangle$ is constant through the boundary layer and is a function of $x$ only. The closure problem appears again in the two-dimensional turbulent boundary layer due to the two additional unknown terms, $\left\langle u^{\prime} v^{\prime}\right\rangle$ and $\left\langle v^{\prime} \theta^{\prime}\right\rangle$, in equations (2.42) and (2.43),respectively.

\subsubsection{Boundary layer scalings}

For boundary layer problems, an important concept is the self similarity which means that from one streamwise position $x$ to another, the velocity profiles look similar and the same holds for the scalar profile. By proper scaling, all the profiles at different downstream positions collapse onto a single curve. According to Barenblatt (1996), the first application was made by Joseph Fourier in 1822 in the context of heat conduction.

For a turbulent boundary layer flow, one always seeks the similarity solutions for the mean velocity and mean scalar profiles in the inner and outer regions of the boundary layer separately. These two regions are of different characteristics. The individual terms in equation (2.42) and (2.43) are of different importance in these two regions of the turbulent boundary layer, e.g. the convection terms are significant in the outer region while the viscous and the scalar flux terms are only important in the inner region.

\section{The inner region scaling}

The behaviour of the flow in the near wall region is of great importance because many features of the flow of engineering significance are determined in the inner layer. The inner layer was first treated in the work by Ludwig Prandtl. The boundary layer equation for the flow field in the inner part simplified from the equation (2.42) reads

$$
0=\nu \frac{\partial^{2}\langle u\rangle}{\partial y^{2}}-\frac{\partial}{\partial y}\left\langle u^{\prime} v^{\prime}\right\rangle
$$

Integrating equation (2.45) we get

$$
\frac{\tau}{\rho} \equiv \nu \frac{\partial\langle u\rangle}{\partial y}-\left\langle u^{\prime} v^{\prime}\right\rangle=\left.\left(\nu \frac{\partial\langle u\rangle}{\partial y}-\left\langle u^{\prime} v^{\prime}\right\rangle\right)\right|_{y=0}=\left.\nu \frac{\partial\langle u\rangle}{\partial y}\right|_{y=0} \equiv \frac{\tau_{w}}{\rho} .
$$

Note that the Reynolds stress $\left\langle u^{\prime} v^{\prime}\right\rangle$ disappears at the wall due to the noslip boundary condition, $\tau$ is the total mean shear stress and $\tau_{w}$ is the total mean shear stress at the wall.

We define the characteristic length scale in the inner region to be

$$
l_{*} \equiv \frac{\nu}{u_{\tau}},
$$


where $u_{\tau}$ is the friction velocity and defined as

$$
u_{\tau} \equiv \sqrt{\frac{\tau_{w}}{\rho}}=\sqrt{\left.\nu \frac{\partial\langle u\rangle}{\partial y}\right|_{y=0}} .
$$

The wall-normal distance expressed in viscous scaling, also called inner scaling or wall units, becomes

$$
y^{+} \equiv \frac{y}{l_{*}}=\frac{y u_{\tau}}{\nu} .
$$

Using viscous scaling, the mean streamwise velocity can be written in the form

$$
u^{+} \equiv \frac{\langle u\rangle}{u_{\tau}}=\Phi_{1}\left(y^{+}\right) .
$$

Equation (2.50) is referred to as the law of the wall. Here we assume outer geometrical restrictions, i.e. the outer length scale to be of negligible influence sufficiently close to the wall, and assume the velocity and Reynolds shear stress profiles to be functions of $y^{+}$only rather than $y^{+}$and $R e$ which is defined by $R e=\frac{U L}{\nu}$ where $U$ denotes the velocity scale, $L$ the length scale and $\nu$ is the kinematic viscosity. Thus the law of the wall will be universal, i.e. the function $\Phi_{1}$ will be the same mathematical function for all flows at sufficiently high Reynolds number. Under such circumstances, the law of the wall is found to agree well with measurements and simulations for $y<0.3 \delta$, where $\delta$ is the boundary layer thickness. Usually we define the thickness of the boundary layer to be at the position where streamwise velocity $u$ reaches the $99 \%$ of the free-stream velocity $U_{\infty}$

$$
u\left(y=\delta_{99 \%}\right)=0.99 U_{\infty} .
$$

Similarly as the mean velocity, the Reynolds shear stress using viscous scaling can be written in the form

$$
\left\langle u^{\prime} v^{\prime}\right\rangle^{+} \equiv-\frac{\left\langle u^{\prime} v^{\prime}\right\rangle}{u_{\tau}^{2}}=\Phi_{2}\left(y^{+}\right),
$$

which is found to hold for $y<0.1 \delta$ when the Reynolds number of the flow is very high.

Using viscous scaling, the equation (2.46) can be written as

$$
\tau^{+} \equiv \frac{d u^{+}}{d y^{+}}-\left\langle u^{\prime} v^{\prime}\right\rangle^{+}=1
$$

or

$$
\Phi_{1}^{\prime}\left(y^{+}\right)+\Phi_{2}\left(y^{+}\right)=1 .
$$

Very close to the wall, the viscous effects are expected to be dominant so that the viscous stress becomes much larger than the Reynolds shear stress. 
This region is the so-called viscous sub-layer. In this layer equation (2.45) (after neglecting the Reynolds shear stress term) can be integrated to yield

$$
u^{+}=y^{+} .
$$

This relation implies that the velocity profile is a linear function of only one variable, which in turn depends on both $x$ and $y$. This linear relation is found to be valid for $y^{+}<5$, see e.g. Pope (2000).

In the case of the scalar, we expect a similar behaviour as for the velocity field. By the same reasoning as above, the boundary layer equation for the scalar field in the inner part simplified from equation (2.43) reads

$$
0=\alpha \frac{\partial^{2}\langle\theta\rangle}{\partial y^{2}}-\frac{\partial\left\langle v^{\prime} \theta^{\prime}\right\rangle}{\partial y} .
$$

Similarly, the law of the wall for scalar field reads

$$
\theta^{+} \equiv \frac{\theta_{w}-\langle\theta\rangle}{\theta_{\tau}}=\Phi_{\theta}\left(y^{+}, \operatorname{Pr}\right)
$$

where function $\Phi_{\theta}$ is a universal function which is dependent on $y^{+}$and the molecular Prandtl number $\operatorname{Pr}$ which is defined by $\operatorname{Pr}=\frac{\nu}{\alpha}$ where $\nu$ is the kinematic viscosity and $\alpha$ the scalar molecular diffusivity. $\theta_{w}$ is the scalar concentration at the wall. $\theta_{\tau}$ is called friction scalar or friction temperature if $\theta$ is considered to be a temperature. According to Cebeci and Bradshaw (1984), the friction temperature is analogous to the friction velocity $u_{\tau}$ and defined by

$$
\theta_{\tau} \equiv \frac{q_{w}}{\rho c_{p} u_{\tau}}
$$

where $\rho$ is the density of the fluid, $c_{p}$ is the heat capacity of the fluid, $u_{\tau}$ is the friction velocity and $q_{w}$ is the rate of the heat transfer from the wall to the flow which is defined by

$$
q_{w}=-\left.k \frac{d\langle\theta\rangle}{d y}\right|_{y=0}
$$

where $k$ is the thermal conductivity and this equation is referred to as the heat conduction law or Fourier's law.

Analogously, very close to the wall, a conductive sub-layer exists for the scalar field as a viscous sub-layer for the velocity field. From the heat conduction law, we can derive the equation for the conductive sub-layer

$$
\theta^{+}=\operatorname{Pr} y^{+} \text {. }
$$

This linear relation is found to be valid for $\operatorname{Pr} y^{+}<3$ (Cebeci and Bradshaw, 1984). 


\section{The outer region}

The turbulent boundary layer is not expected to explicitly depend on the viscosity $\nu$ in the outer region, thus the viscous stress is to be negligible. Hence, equation (2.42) can be reduced to

$$
\langle u\rangle \frac{\partial\langle u\rangle}{\partial x}+\langle v\rangle \frac{\partial\langle u\rangle}{\partial y}=-\frac{\partial\left\langle u^{\prime} v^{\prime}\right\rangle}{\partial y} .
$$

The pressure term vanished since we are dealing with a zero pressure gradient (ZPG) case, i.e. $\frac{d\langle p\rangle}{d x}=0$.

We define the characteristic length scale in the outer region to be

$$
\Delta \equiv \delta^{*} .
$$

where the $\delta^{*}$ is the displacement thickness defined by

$$
\delta^{*}=\int_{0}^{\infty}\left(1-\frac{\langle u\rangle}{U_{\infty}}\right) \mathrm{d} y .
$$

The outer scaling for the wall-normal distance is formed as

$$
\eta \equiv \frac{y}{\Delta}=\frac{y}{\delta^{*}} .
$$

The gross characteristic of the turbulence in the outer part of the turbulent boundary layer is inviscid, and the Reynolds shear stress in the outer region results in a drag on the flow and generates a velocity defect $\left(\langle u\rangle-U_{\infty}\right)$, which is expected to be proportional to the wall friction characterised by $u_{\tau}$. Therefore, the mean velocity is scaled as

$$
\frac{\langle u\rangle-U_{\infty}}{u_{\tau}}=\Psi_{1}(\eta),
$$

which is called the velocity defect law and the Reynolds shear stress is scaled as

$$
-\frac{\left\langle u^{\prime} v^{\prime}\right\rangle}{u_{\tau}^{2}}=\Psi_{2}(\eta)
$$

If the velocity scale for the scaling of the Reynolds shear stress is chosen as another variable, consistency would require that the ratio $\frac{u_{\tau}}{U_{\infty}}$ has to be assumed to approach a constant at infinite high Reynolds number (Österlund, 1999).

The equation (2.43) of scalar field in the outer region can be reduced to

$$
\langle u\rangle \frac{\partial\langle\theta\rangle}{\partial x}+\langle v\rangle \frac{\partial\langle\theta\rangle}{\partial y}=-\frac{\partial\left\langle v^{\prime} \theta^{\prime}\right\rangle}{\partial y} .
$$

Similar consideration leads to the defect law for the scalar

$$
\frac{\langle\theta\rangle-\theta_{\infty}}{\theta_{\tau}}=\Psi_{\theta}(\eta)
$$

where $\theta_{\infty}$ is the scalar concentration in the free-stream and $\theta_{\tau}$ the friction scalar. 


\section{The overlap region}

From the analysis above, we know that the velocity and scalar fields are governed by different laws (equations (2.50), (2.52), (2.57), (2.65), (2.66), $(2.68))$ in the inner and outer regions of the boundary layer. However at high enough Reynolds number, the outer edge of the inner layer can overlap the inner edge of the outer layer. This means the behaviour of equations $(2.50),(2.52)$ and $(2.57))$ for $y^{+} \rightarrow \infty$ should coincide with that given by the equations (2.65), (2.66) and (2.68) for $\eta \rightarrow 0$. Hence in the so-called overlap region where $l_{*} \ll y \ll \delta^{*}$, all the equations are valid simultaneously. The classical treatment is a matching of the equations involving both inner and outer regions. Following the derivation by Tennekes and Lumley (1972), we obtain

$$
\begin{gathered}
\Phi_{1}\left(y^{+}\right)=\frac{\langle u\rangle}{u_{\tau}}=\frac{1}{\kappa} \ln \left(y^{+}\right)+A, \\
\Psi_{1}(\eta)=\frac{\langle u\rangle-U_{\infty}}{u_{\tau}}=\frac{1}{\kappa} \ln (\eta)+B,
\end{gathered}
$$

where $\kappa$ is called the Kármán constant. The constants $\kappa \approx 0.41, A \approx 5.2$ and $B \approx 1$ can be found from the experiments.

Equations (2.69) and (2.70) are the velocity distribution in the overlap region and also called logarithmic law or log-law for short. The value of the Kármán constant, $\kappa$, is set to 0.41 throughout this thesis. Österlund et al. (2000) proposed a value for the Kármán constant of 0.38 for large enough Reynolds number. However, Spalart (1988) has shown that the traditional value of 0.41 gives good agreement for lower Reynolds numbers.

The log-law for the scalar field takes the same form as the velocity field

$$
\begin{gathered}
\Phi_{\theta}\left(y^{+}, \operatorname{Pr}\right)=\frac{\theta_{w}-\langle\theta\rangle}{\theta_{\tau}}=\frac{1}{\kappa_{\theta}} \ln \left(y^{+}\right)+A_{\theta}(\operatorname{Pr}), \\
\Psi_{\theta}(\eta)=\frac{\theta_{\infty}-\langle\theta\rangle}{\theta_{\tau}}=\frac{1}{\kappa_{\theta}} \ln (\eta)+B_{\theta}(\operatorname{Pr}),
\end{gathered}
$$

where the Kármán constant for the scalar field, $\kappa_{\theta}=0.33$ (Wikström, 1998). Both $A_{\theta}$ and $B_{\theta}$ are functions of $P r$. It might be more logical to write the argument of the logarithm as $\mathrm{Pry}^{+}$, that is, incorporating the scalar conductivity rather than the viscosity, but this will merely change the constant $A_{\theta}$ without eliminating it.

A typical mean streamwise velocity profile of a turbulent boundary layer denoted by the open circle, is shown in Figure 2.1. As we can see the DNS data fits the log-law very well in the log region. In the outer region of the boundary layer, a departure is observed. The outer region in which there is a departure from the law of the wall is frequently called the wake region. There are several parameterisations available for the wake region, e.g. the wake function proposed by Coles (1956). However they are completely empirical. 
For the profile of the mean scalar, it is essentially the same as for the mean streamwise velocity. It is anticipated that a wake region for the mean scalar profile also does exist.

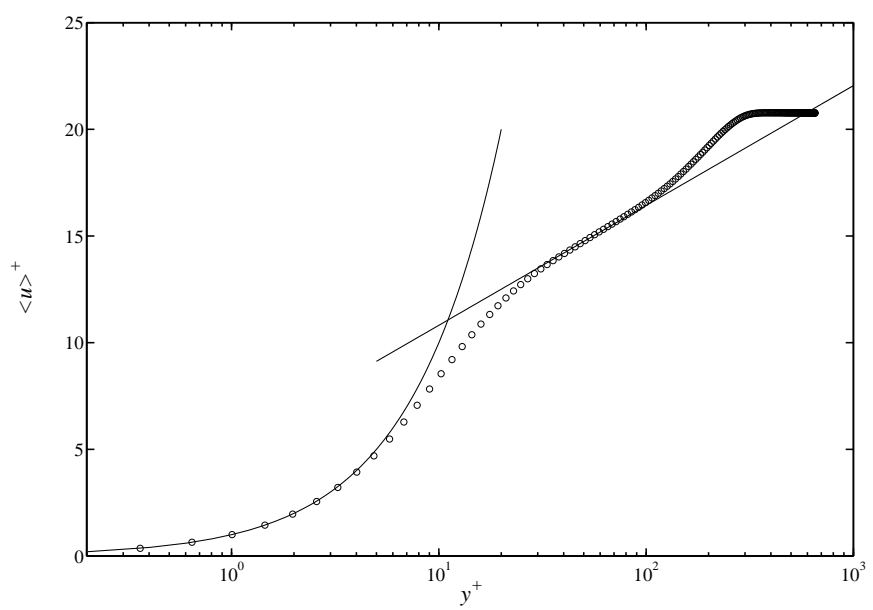

Figure 2.1: Mean streamwise velocity profile. —L Log-law, o DNS.

One should always bear in mind that we assume that $\alpha \frac{\partial\langle\theta\rangle}{\partial y}$ is dominant in the inner region while $\left\langle v^{\prime} \theta^{\prime}\right\rangle$ is dominant in the outer region. However this might be inaccurate since $\alpha \frac{\partial\langle\theta\rangle}{\partial y}$ can vary tremendously with the molecular Prandtl number which is depended on the particular fluid. Prandtl numbers for viscous fluids like oils can easily exceed 100, while those for liquid metals can be as low as 0.001. We know that in the inner and outer regions, the terms neglected are not zero, they are merely small compared to the other ones. By choosing a different Prandtl number, the situation may be reversed. For the present we would like to neglect $\left\langle v^{\prime} \theta^{\prime}\right\rangle$ in the inner region and $\alpha \frac{\partial\langle\theta\rangle}{\partial y}$ in the outer region. This means we are restricted to $\mathcal{O}(\operatorname{Pr})=1$. The use of the $\Phi_{\theta}$ in equation (2.57) which is a function of $y^{+}$and $\operatorname{Pr}$ is a way of compensating for this effect. For more details, see e.g. Kays and Crawford (1993). 


\section{Chapter 3}

\section{Direct Numerical Simulation}

\subsection{Non-dimensional Equations}

It is often convenient to work with a non-dimensional form of the NavierStokes equations and the scalar transport equation. The non-dimensional form of the variables are

$$
\begin{gathered}
u_{i}^{\star}=\frac{u_{i}}{U_{\infty}}, x_{i}^{\star}=\frac{x_{i}}{\delta_{0}^{*}}, p^{\star}=\frac{p}{\rho U_{\infty}^{2}}, t^{\star}=\frac{t U_{\infty}}{\delta_{0}^{*}}, \\
\theta^{\star}=\frac{\Theta-\Theta_{\infty}}{\Theta_{w}-\Theta_{\infty}}, \text { for the isothermal boundary condition } \\
\theta^{\star}=\frac{k\left(\Theta-\Theta_{\infty}\right)}{q_{w} \delta_{0}^{*}}, \quad \text { for the isoflux boundary condition }
\end{gathered}
$$

where $\star$ signifies a non-dimensional variable. $x_{i}$ is the coordinates. $U_{\infty}$ is the undisturbed laminar streamwise free-stream velocity at $x=0$ and $t=0$ as the velocity scale, $\delta_{0}^{*}$ is the displacement thickness of the undisturbed streamwise velocity at $x=0$ and $t=0 . \rho$ is the density of the fluid. The reference scalar concentrations are chosen to be the scalar concentration at the wall $\Theta_{w}$ and the one in the free-stream $\Theta_{\infty} . q_{w}$ is the rate of the scalar transfer from the wall to the flow defined by equation (2.59) and $k$ is the scalar conductivity. Unless specified otherwise the superscript $\star$ will be dropped from the non-dimensional variables for simplicity.

The non-dimensional governing equations are given in the following form

$$
\begin{gathered}
\frac{\partial u_{i}}{\partial t}+u_{j} \frac{\partial u_{i}}{\partial x_{j}}=-\frac{\partial p}{\partial x_{i}}+\frac{1}{\operatorname{Re}} \frac{\partial^{2} u_{i}}{\partial x_{j} \partial x_{j}}, \\
\frac{\partial u_{i}}{\partial x_{i}}=0 \\
\frac{\partial \theta}{\partial t}+u_{i} \frac{\partial \theta}{\partial x_{i}}=\frac{1}{\operatorname{RePr}} \frac{\partial^{2} \theta}{\partial x_{i} \partial x_{i}},
\end{gathered}
$$


where the Reynolds number $R e$ is based on the free-stream velocity $U_{\infty}$ and the inlet displacement thickness $\delta_{0}^{*}, \operatorname{Pr}$ is the molecular Prandtl number. The buoyancy effect and fluid property variation with the scalar are neglected, i.e. the scalar is regarded as a passive scalar. The multiplication of $\operatorname{RePr}$ is another non-dimensional number, named Péclet number $(\mathrm{Pe})$. This dimensionless number represents the ratio of convective transport of scalar to scalar molecular diffusion.

\subsection{Boundary Conditions}

The velocity and scalar fields are periodic in the horizontal directions whereas specified boundary conditions at the wall and in the free-stream are needed to solve the governing equations.

\subsubsection{Boundary conditions for flow field}

At the wall, the no-slip boundary conditions read, i.e. the velocity of the fluid at a solid surface must be equal to the velocity of the surface,

$$
\left.u\right|_{y=0}=0,\left.v\right|_{y=0}=0,\left.w\right|_{y=0}=0
$$

and

$$
\left.\frac{\partial v}{\partial y}\right|_{y=0}=0
$$

which is derived from the continuity equation.

The flow is assumed to extend to an infinite distance perpendicular to the plate, but due to the impracticability of discretizing a infinite domain a truncated domain has to be considered. At the upper boundary in the freestream an artificial boundary condition is applied. The simplest possible boundary condition is a Dirichlet condition, i.e.

$$
\left.u_{i}\right|_{y=y_{L}}=\left.\mathcal{U}_{i}\right|_{y=y_{L}}
$$

where $y_{L}$ is the height of the solution domain in the wall-normal direction

in physical space. The size of the solution domain in the streamwise and spanwise directions are denoted by $x_{L}$ and $z_{L}$, respectively. $\mathcal{U}_{i}(x, y)$ is a laminar base flow that is chosen as the Blasius flow for the present case.

However, the desired flow solution generally contains a disturbance and that will be forced to zero by the Dirichlet condition. This introduces an error compared to the exact solution for which the boundary condition is applied at an infinite distance from the wall. The error may result in increased damping of disturbances in the boundary layer (Lundbladh et al., 1999). Some improvement can be made by imposing a Neumann condition

$$
\left.\frac{\partial u_{i}}{\partial y}\right|_{y=y_{L}}=\left.\frac{\partial \mathcal{U}_{i}}{\partial y}\right|_{y=y_{L}},
$$

which is implemented in the present DNS code. 


\subsubsection{Boundary conditions for scalar field}

In the present implementation for the scalar field, we use two types of wall boundary conditions. One is an isothermal wall and the other is an isoflux wall. The boundary conditions for these two walls are given by

$$
\begin{array}{r}
\left.\theta\right|_{y=0}=1, \text { for the isothermal boundary condition } \\
\left.\frac{\partial \theta}{\partial y}\right|_{y=0}=-1, \quad \text { for the isoflux boundary condition }
\end{array}
$$

These two kinds of wall boundary conditions are actually two limiting cases of the physical configuration. Considering $\theta$ to be the temperature concentration, the isothermal wall boundary condition corresponds to a situation where the fluid with negligible density $\rho$, heat capacity $c_{p}$ and thermal conductivity $k$ is heated by an infinitely thick wall with large density $\rho_{w}$, heat capacity $c_{p w}$ and thermal conductivity $k_{w}$. Hence the thermal activity ratio

$K$ defined by $\sqrt{\frac{\rho c_{p} k}{\rho_{w} c_{p w} k_{w}}}$ is vanishing in this case. The isoflux wall boundary condition, however, is an opposite case of the isothermal one. It signifies an extremely thin wall with small density, heat capacity and thermal conductivity while the corresponding fluid properties are quite large. This leads to a thermal activity ratio $K$ of $\infty$ (Tiselj et al., 2001a). In order to reveal the details of the heat transfer near the wall for a more general case, one has to solve the conjugate heat transfer problem with a given thickness, material properties of the solid wall and the properties of the fluid. For more details about conjugate heat transfer, see e.g. Kasagi et al. (1989).

The boundary condition in the free-stream is

$$
\left.\theta\right|_{y=y_{L}}=0
$$

\subsection{Computational Domain}

In this thesis we are considering a turbulent boundary layer flow over a flat plate with a passive scalar under a uniform free-stream with zero pressure gradient (ZPG). The simulation starts with a laminar Blasius boundary layer at the inlet $\left(R e_{x}=68450\right.$ where $R e_{x}$ is the Reynolds number based on the streamwise position $x$ and the free-stream velocity $U_{\infty}$ ), then the laminar flow is tripped by a random volume force strip to transition and finally becomes turbulent. The trip forcing in the present case is located at the wall with streamwise position at $x=10$ corresponding to $R e_{x}=72950$.

The computational domain consists of a three dimensional cuboid bounded at the lower edge by a flat plate with no-slip boundary condition. The boundary layer will grow in the domain starting with an initial boundary layer thickness $\delta_{0}^{*}$. In the streamwise direction the computational domain is bounded by the fringe region, which will be described in Section 3.4.3. 
All the relevant quantities of the flow are scaled with the inlet boundary layer displacement thickness $\delta_{0}^{*}$, the free-stream velocity $U_{\infty}$ and the viscosity $\nu$, i.e. the same dimensions used by Skote (2001) and Schlatter (2001). The Reynolds number based on the inlet displacement thickness and freestream velocity is set to be 450 . The distance from the leading edge $x_{0}$ can then be computed from

$$
\frac{x_{0}}{\delta_{0}^{*}}=\frac{R e_{0}^{*}}{1.72^{2}}=152.11
$$

which corresponds to $R e_{x}=68450$ or $R e_{\theta}=175$. The simulation is carried out in a computational box with the length $x_{L}$, height $y_{L}$ and width $z_{L}$ being $750 \delta_{0}^{*}, 40 \delta_{0}^{*}$ and $34 \delta_{0}^{*}$. The streamwise extent of the box must for spatially developing flows include the length of the fringe region which typically accounts for around $10 \%$ of the total length of the computational domain. In the present case the fringe length is chosen to be 90 . The vertical extent of the box must include the whole boundary layer. Depending on the choice of free-stream boundary condition, the box may include only the boundary layer or a few times more. The sufficiency of the box height may be investigated through numerical experiments.

We use $1024 \times 289 \times 128$ grid points in the streamwise, wall-normal and spanwise directions, respectively. The grid spacing is uniform in the streamwise and spanwise directions. A Gauss-Lobatto distribution is used in the wall-normal direction. The Gauss-Lobatto collocation points are

$$
y_{i}=\cos \left(\pi \frac{i}{N_{y}}\right), i=0,1,2, \ldots, N_{y} .
$$

This gives the spacing to be of order $N_{y}^{-2}$ near the wall, and in the middle of the computational box to be of order $N_{y}^{-1}$ where $N_{y}$ is the number of grid points in the wall-normal direction, see e.g. Canuto et al. (1988) for details. The grid spacings in the wall units are $\Delta x^{+} \approx 17, \Delta z^{+} \approx 6.3$, $\Delta y_{\min }^{+} \approx 0.025$ at the wall and $\Delta y_{\max }^{+} \approx 4.6$ at the edge of the boundary layer based on the friction velocity at the $x=150$ of the computational box where the flow becomes turbulent.

We use an adaptive computational time stepping for this DNS. The time step is regulated to keep the Courant-Friederichs-Lewy (CFL) number close to the maximum CFL which is calculated from the velocities in physical space and set to $0.9 \sqrt{8}$ for the four stage Runge-Kutta method. For more details see Lundbladh et al. (1999) or Schlatter (2001). The code was compiled for 64 processors (parallel vector processors with distributed memory). The average time step size $\Delta t$ is about 0.02378 which corresponds to around 40 seconds in wall-clock time. Statistics are sampled at every 32 time steps and averaged for about 1000 time units $\frac{\delta_{0}^{*}}{U_{\infty}}$. 


\subsection{Numerical Method}

\subsubsection{Numerical scheme}

The numerical code used for the calculations presented in this thesis has been developed and improved during the past years at KTH Stockholm. The code solves the incompressible Navier-Stokes equations and can be run on parallel computers, using OpenMP (Multi Processing) or MPI (Message passing interface) with shared or distributed memory. It was applied successfully in a number of works by a number of users on a variety of workstations and super computers, see e.g. Lundbladh et al. (1999) or Skote (2001). The following description is partly taken from Lundbladh et al. (1999), Skote (2001) and Schlatter (2001).

The direct numerical simulation has been performed with the spectral algorithm described in detail in Lundbladh et al. (1999). In a spectral method the solution is approximated by an expansion in smooth (global) functions, e.g. trigonometric functions as in our case, which provides a fast convergence rate to the approximation of the real solution, i.e. the so-called spectral convergence. The earliest applications to partial differential equations were developed by Kreiss and Oliger (1972) and Orszag (1972), who named the method pseudo-spectral. The term pseudo-spectral refers to the multiplications of the non-linear terms, which are calculated in physical space to avoid the evaluation of convolution sums. The transformation between physical and spectral space can be efficiently done by Fast Fourier Transform (FFT) algorithms that became generally known in the 1960's.

The high accuracy in spectral methods compared to finite element or finite difference discretizations is the result of the fast convergence rate of spectral approximations to a function mentioned above. Efficient implementations of pseudo-spectral methods can be made thanks to the lower costs of performing FFTs. Moreover, the data structure makes the algorithms suitable for both vectorization and parallelization. However, the spectral approximation in single domain limits the applications to rather simple geometries.

\subsubsection{Discretization}

The algorithm used for solving the equations (3.2), (3.4) and (3.3) is similar to that for channel geometry of Kim et al. (1987), using Fourier series expansion in the wall parallel (streamwise and spanwise) directions and Chebyshev series in the wall-normal direction using the Chebyshev tau method (CTM).

The time advancement used is a four-step low storage third-order RungeKutta method for the non-linear terms and a second-order Crank-Nicolson method for the linear terms. The non-linear terms are calculated in physical space rather than spectral space (pseudo-spectral method). Aliasing errors from the evaluation of the non-linear terms are removed by the $3 / 2$-rule when 
the horizontal FFTs are calculated. Although no rigorous stability criterion for the Navier-Stokes equations integrated by a Runge-Kutta scheme exists, the stability analysis for the four stage Runge-Kutta method shows a stability limit of $\mathrm{CFL}=\sqrt{8}$. This is used in the adaptive time stepping to calculate the maximal stable time step size $\Delta t$.

The numerical code is written in FORTRAN 77 and consists of two major parts. One linear part where the equations are solved in spectral space, and one non-linear part where the non-linear terms in the equations are computed in physical space. The linear part needs data for one spanwise position at a time since the equations are solved in the wall-normal direction. The non-linear part needs data for one $y$ position at a time since the FFT is performed in the horizontal (streamwise and spanwise) directions. The flow variables are stored at an intermediate level with spectral representation in the horizontal directions and physical representation in the wall-normal direction. All spatial derivatives are calculated with spectral accuracy. The main computational effort in these two parts is in the FFT.

\subsubsection{Fringe region}

For the present DNS, since the boundary layer is developing in the downstream direction, it is necessary to use non-periodic boundary conditions in the streamwise direction. So a fringe region, similar to that described by Nordström et al. (1999), is added downstream of the physical domain to retain periodic boundary conditions for the Fourier discretization. In the fringe region, the disturbances are damped and the flow is forced from the outflow of the physical domain to the inflow. In this way the physical domain and the fringe region together satisfy periodic boundary conditions. The fringe region is implemented by the addition of a volume force $F$ into the Navier-Stokes equations

$$
\frac{\partial u_{i}}{\partial t}+u_{j} \frac{\partial u_{i}}{\partial x_{j}}=-\frac{1}{\rho} \frac{\partial p}{\partial x_{i}}+\nu \frac{\partial^{2} u_{i}}{\partial x_{j} \partial x_{j}}+F_{i}
$$

The forcing term is

$$
F_{i}=\lambda(x)\left(\mathcal{U}_{i}-u_{i}\right),
$$

where $\lambda(x)$ is the strength of the forcing. $\lambda(x)$ is a non-negative fringe function which is significantly non-zero only within the fringe region and designed to minimize the upstream influence. $\mathcal{U}_{i}$ is the same base flow field used for the initial calculation and the boundary conditions, which also contains the desired flow solution in the fringe. The streamwise velocity component of $\mathcal{U}_{i}$ is calculated as

$$
\mathcal{U}_{x}(x, y)=\tilde{u}(x, y)+\left[\tilde{u}\left(x+x_{L}, y\right)-\tilde{u}(x, y)\right] S\left(\frac{x-x_{m i x}}{\Delta_{m i x}}\right)
$$


where $\tilde{u}(x, y)$ is normally a solution to the boundary layer equations. $x_{L}$ denotes the extent of the computational domain. $x_{m i x}$ and $\Delta_{m i x}$ are chosen so that the prescribed flow within the fringe region smoothly changes from the outflow velocity of the physical domain to the desired inflow velocity. $S$ is a smooth step function described in equation (3.17). The wall-normal component $\mathcal{U}_{y}$ is then calculated from the equation of continuity, and the spanwise velocity $\mathcal{U}_{z}$ is set to zero for the present simulation since the base flow is two dimensional.

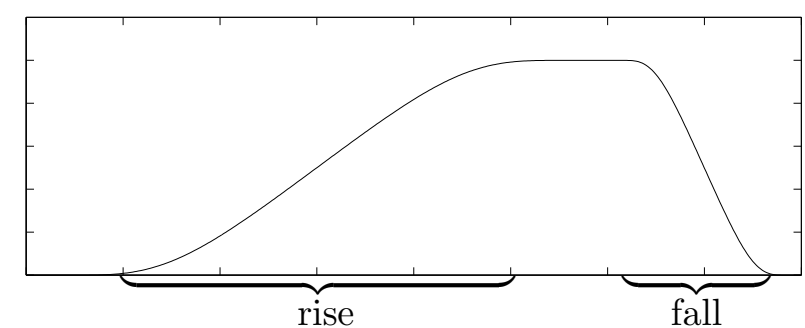

Figure 3.1: A sketch of the fringe function $\lambda(x)$.

A convenient form of the fringe function $\lambda$ is described as follows

$$
\lambda(x)=\lambda_{\text {max }}\left[S\left(\frac{x-x_{\text {start }}}{\Delta_{\text {rise }}}\right)-S\left(\frac{x-x_{\text {end }}}{\Delta_{\text {fall }}}+1\right)\right],
$$

where $\lambda_{\max }$ is the maximum strength of the damping. $x_{\text {start }}$ to $x_{\text {end }}$ is the spatial extent of the region where the damping function is non-zero and $\Delta_{\text {rise }}$ and $\Delta_{\text {fall }}$ denote the rise and fall distance of the damping function. See Figure 3.1 for detail. $S(x)$ is a smooth step function rising from zero for negative $x$ to one for $x \geq 1$. We have used the following form for $\mathrm{S}$, which has the advantage of having continuous derivatives of all orders for $x \neq 0$ and $x \neq 1$

$$
S(x)=\left\{\begin{array}{ll}
0 & x \leq 0 \\
1 /\left[1+\exp \left(\frac{1}{x-1}+\frac{1}{x}\right)\right] & 0<x<1 \\
1 & x \geq 1
\end{array} .\right.
$$

To achieve maximum damping both the total length of the fringe and $\lambda_{\max }$ have to be tuned. The actual shape of $\lambda(x)$ is less important for the damping but it should have its maximum closer to $x_{\text {end }}$ other than to $x_{\text {start }}$. See Nordström et al. (1999) for an investigation of the fringe region technique.

Figure 3.2 illustrates the variation of the boundary layer thickness and the mean flow profile in the computational box as well as a typical fringe function $\lambda(x)$. Note that the analysis presented above also applies for the scalar field. 


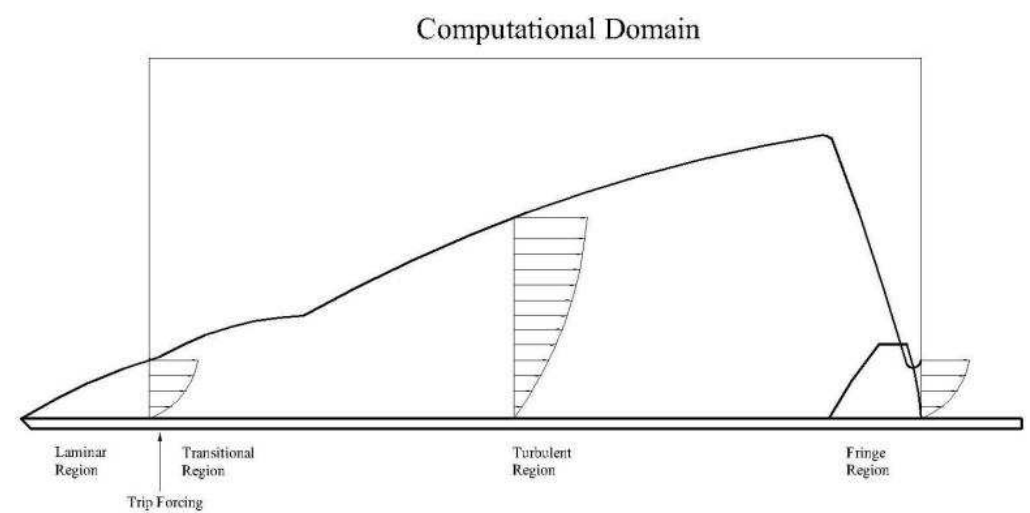

Figure 3.2: The boundary layer thickness $\delta$ of a flow that grows downstream in the physical domain and is reduced in the fringe region by the forcing. The flow profile is returned to the desired inflow profile in the fringe region, where the fringe function $\lambda(x)$ is non-zero.

\subsection{Base Flow}

To start the simulation, initial velocity and scalar profiles must first be generated which are also used in the fringe region as base flow. In the present simulation a Blasius velocity profile is chosen to be the base flow. It is a similarity laminar boundary layer profile derived from the laminar boundary layer equations for flow over a flat plate which is a special case of the Falkner-Skan equations. The Falkner-Skan equations read

$$
f^{\prime \prime \prime}+\frac{m+1}{2} f f^{\prime \prime}+m\left[1-\left(f^{\prime}\right)^{2}\right]=0
$$

and

$$
\frac{1}{P r} g^{\prime \prime}+\frac{m+1}{2} f g^{\prime}+n(1-g) f^{\prime}=0,
$$

where $f$ and $g$ are the dimensionless stream function and scalar function and defined by

$$
f^{\prime}(\eta)=\frac{u}{U_{\infty}}
$$

and

$$
g(\eta)=\frac{\Theta-\Theta_{\infty}}{\Delta T}
$$

respectively and $\Delta T$ is defined by

$$
\begin{gathered}
\Delta T=\Theta_{w}-\Theta_{\infty}, \quad \text { for the isothermal boundary condition } \\
\Delta T=\frac{q_{w} \delta_{0}^{*}}{k}, \quad \text { for the isoflux boundary condition }
\end{gathered}
$$

which is consistent with the non-dimensional scaling in equation (3.1). $U_{\infty}$ is the laminar streamwise free-stream velocity at $x=0$ and $t=0, \delta_{0}^{*}$ is the 
displacement thickness of the undisturbed streamwise velocity at $x=0$ and $t=0 . \Theta_{w}$ and $\Theta_{\infty}$ are the reference scalar concentrations at the wall and in the free-stream. $q_{w}$ is the rate of the scalar transfer from the wall to the flow and $k$ is the scalar conductivity. $f$ and $g$ are functions of $\eta$ only where $\eta$ is the similarity variable and depends on both $x$ and $y$. A prime denotes the derivative with respect to $\eta$. The constants $m$ and $n$ in equations (3.18) and (3.19) are defined by

$$
m=\frac{x}{U_{\infty}} \frac{d U_{\infty}}{d x}
$$

and

$$
n=\frac{x}{\Delta T} \frac{d \Delta T}{d x} .
$$

In the present DNS with zero pressure gradient (ZPG), both $m$ and $n$ are 0 for the isothermal boundary condition while $m=0$ and $n=0.5$ for the isoflux boundary condition. For more details, see e.g. Schlichting (1987) or Cebeci and Bradshaw (1984).

The base flow can either be parallel or space developing. For a spatially developing flow as in our case the base flow from the upstream and the downstream end are blended in the fringe region. The start and blending length must be specified. Typically the start is given as a negative number, i.e. the distance upstream of the inflow boundary where the blend starts is given. Here we choose $x=-90$ for the start of blending.

To examine whether the base flow is converged or not, we ran a test case without imposing any disturbances. The simulation was run for a total of 5000 time units $\frac{\delta_{0}^{*}}{U_{\infty}}$. The velocity and scalar profiles at the end of the simulation are compared with the initial profiles and the difference is of an order of $10^{-6}$ to $10^{-5}$, so we draw a conclusion that the base flow is really converged and could be used for the simulation. 


\section{Chapter 4}

\section{Averaged Results}

A direct numerical simulation was being carried out during the last few months. A large amount of statistics of both the mean flow and scalar distributions and the corresponding fluctuations was obtained. In this chapter these results and comparisons with other simulations as well as experimental data of different types of flow will be presented. All the quantities are

averaged for a long enough time span of 1000 time units $\frac{\delta_{0}^{*}}{U_{\infty}}$ to make sure that they are converged. However each quantity is only compared at some of the available positions so that the figures will not be too cluttered. The chosen positions may vary from one to the other, but for most of the results, the positions are chosen in the turbulent region. Moreover the results will be compared at the same Reynolds numbers rather than at the same downstream positions, since different experiments and simulations have different flow conditions, so only comparisons at specific values of Reynolds number provide meaningful results.

In Section 4.1, the numerical parameters used in the simulation as well as the relation between different types of Reynolds numbers and the downstream positions are summarised. The hydrodynamic results of the numerical simulation are discussed in Section 4.2 with the scalar transfer results discussed in Section 4.3. At last the comparisons between the flow field and scalar field are made in Section 4.4.

\subsection{Numerical Parameters}

A summary of the major parameters of the simulation and an overview of the relation between different types of Reynolds numbers and downstream positions are listed in Tables 4.1 and 4.2. The variation of $R e_{\delta^{*}}$ and $R e_{\theta}$ with $R e_{x}$ is shown in Figure 4.1. Note that, at the inlet, the flow is still laminar. 


\begin{tabular}{|c|c|}
\hline$R e_{x}$ at inlet $(x=0)$ & 68450 \\
$R e_{\delta^{*}}$ at inlet $(x=0)$ & 450 \\
$R e_{\theta}$ at inlet $(x=0)$ & 175 \\
$R e_{\delta_{99}}$ at inlet $(x=0)$ & 46 \\
\hline$x_{L}$ (with fringe region) & 750 \\
$y_{L}$ & 40 \\
$z_{L}$ & 34 \\
\hline fringe start $x_{\text {start }}$ & 660 \\
fringe end $x_{\text {end }}$ & 750 \\
fringe strength $\lambda_{\text {max }}$ & 1.0 \\
$\Delta_{\text {rise }}$ & 50 \\
$\Delta_{\text {fall }}$ & 15 \\
\hline start of blending & -90 \\
blending length & 75 \\
\hline number of grid points in $x\left(N_{x}\right)$ & 1024 \\
number of grid points in $y\left(N_{y}\right)$ & 289 \\
number of grid points in $z\left(N_{z}\right)$ & 128 \\
\hline
\end{tabular}

Table 4.1: Parameters for the direct numerical simulation.

\begin{tabular}{|c|c|c|c|c|}
\hline$x$ & $R e_{x}$ & $R e_{\delta^{*}}$ & $R e_{\theta}$ & $R e_{\delta_{99}}$ \\
\hline 0 & 68450 & 450 & 175 & 46 \\
100 & 113450 & 537 & 341 & 160 \\
150 & 135950 & 617 & 396 & 180 \\
200 & 158450 & 704 & 455 & 197 \\
250 & 180950 & 788 & 515 & 218 \\
300 & 203450 & 871 & 571 & 236 \\
350 & 225950 & 950 & 628 & 256 \\
400 & 248450 & 1024 & 680 & 271 \\
450 & 270950 & 1094 & 728 & 282 \\
500 & 293450 & 1164 & 779 & 303 \\
550 & 315950 & 1234 & 830 & 316 \\
600 & 338450 & 1286 & 867 & 327 \\
\hline
\end{tabular}

Table 4.2: Various Reynolds numbers with respect to the downstream locations. $x$ denotes the non-dimensional streamwise positions. $R e_{x}, R e_{\delta^{*}}$ and $R_{\theta}$ are the Reynolds numbers based on $x$, displacement thickness $\delta^{*}$, momentum thickness $\theta$ and free-stream velocity $U_{\infty}$ while $R e_{\delta_{99}}$ is based on the $99 \%$ of the boundary layer thickness $\delta_{99}$ and local friction velocity $u_{\tau}$.

\section{$4.2 \quad$ Hydrodynamic Results}

The hydrodynamic results obtained from the DNS are shown in this section. In general, the agreements with other simulation and experimental results 


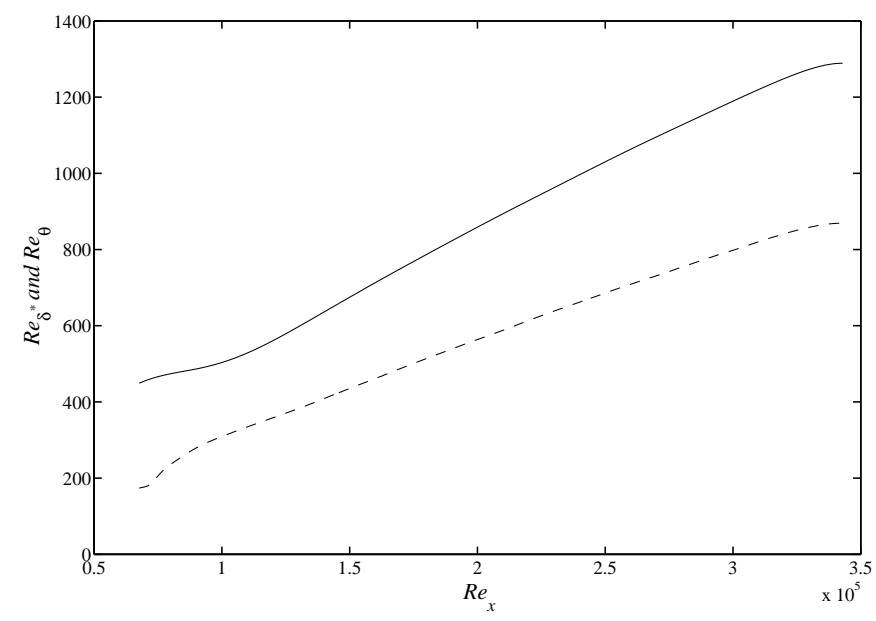

Figure 4.1: Reynolds number based on displacement $R e_{\delta^{*}}$ and momentum thickness $R e_{\theta}$ versus Reynolds number based on downstream positions $R e_{x}$. $-R e_{\delta^{*}},---R e_{\theta}$.

are exceptional for most of the quantities.

\subsubsection{Mean flow results}
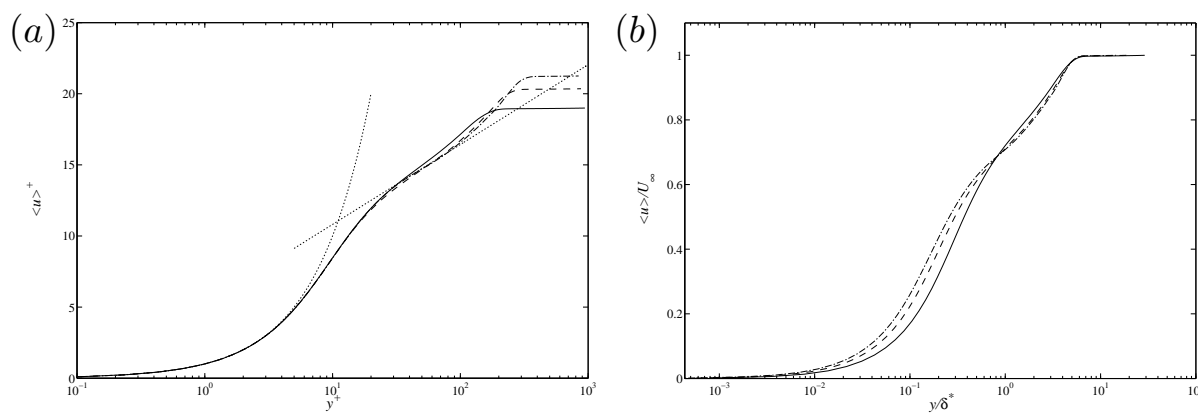

Figure 4.2: Mean streamwise velocity profile at different downstream positions. - at $R e_{\theta}=396,---$ at $R e_{\theta}=628,-$ - at $R e_{\theta}=830, \cdots \cdots$ Loglaw. (a) Inner scaling, (b) Outer scaling.

In Figure 4.2, the mean streamwise velocity profiles at several downstream positions are plotted in inner and outer scaling, respectively. For inner scaling the mean streamwise velocity $\langle u\rangle$ is scaled by $u_{\tau}$ and the wallnormal distance $y$ is scaled by $\frac{\nu}{u_{\tau}}$ where $\nu$ is the kinematic viscosity and $u_{\tau}$ is the friction velocity defined by equation (2.48). For the outer scaling, the mean streamwise velocity $\langle u\rangle$ is scaled by $U_{\infty}$ and the wall-normal distance $y$ is scaled by $\delta^{*}$ where $U_{\infty}$ is the free-stream velocity and $\delta^{*}$ the local displacement thickness. From Figure $4.2(a)$, we see that inside the viscous 
sub-layer, the calculated mean velocity profile follows the relation of

$$
u^{+}=y^{+}
$$

fairly well and the final station profile compares well with the classical loglaw

$$
u^{+}=\frac{1}{\kappa} \ln y^{+}+A,
$$

where $\kappa=0.41$ and $A=5.2$ (Pope, 2000), and the wake region is apparent.

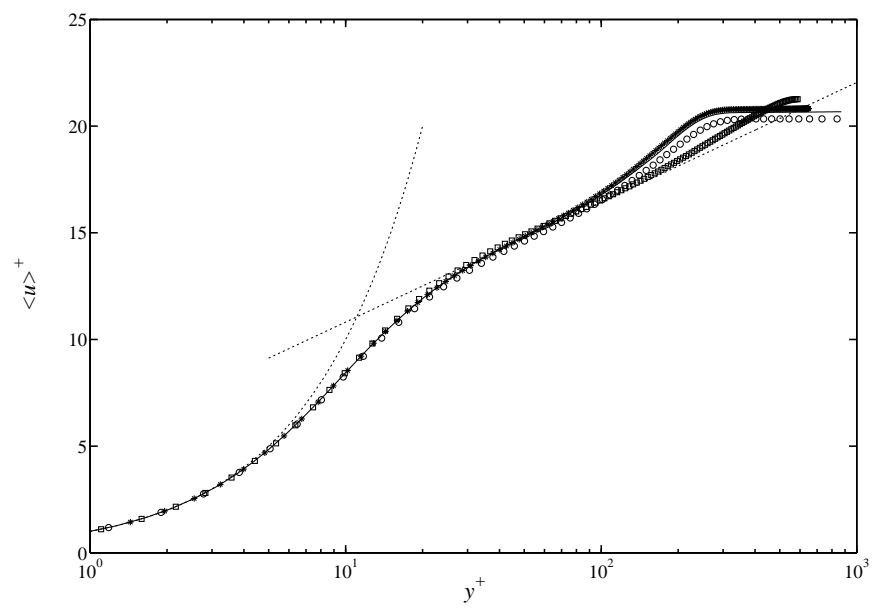

Figure 4.3: Comparison of the mean streamwise velocity $\langle u\rangle$ with other numerical simulations. - Present DNS at $R e_{\theta}=670$, ○ Spalart (1988) at $R e_{\theta}=670, *$ Komminaho and Skote (2002) at $R e_{\theta}=666$, $\square$ Moser et al. (1999) at $R e_{\tau}=590, \cdots \cdots$. Log-law.

Figure 4.3 compares the mean streamwise velocity profile with other DNS data from Spalart (1988), Komminaho and Skote (2002) and Moser et al. (1999). The agreement between the current simulation and other DNS is very good.

Note that the mean velocity profiles rise steeply from the wall and then become flatter away from the wall. This "flatness" of the mean velocity profiles is quantified by the shape factor $H_{12}$, which is defined by

$$
H_{12}=\frac{\delta^{*}}{\theta}=\frac{\delta_{1}}{\delta_{2}},
$$

where $\delta^{*}$ is the displacement thickness and $\theta$ the momentum thickness. They are also denoted as $\delta_{1}$ and $\delta_{2}$, respectively and defined by

$$
\delta^{*} \equiv \delta_{1}=\int_{0}^{\infty}\left(1-\frac{\langle u\rangle}{U_{\infty}}\right) \mathrm{d} y
$$

and

$$
\theta \equiv \delta_{2}=\int_{0}^{\infty} \frac{\langle u\rangle}{U_{\infty}}\left(1-\frac{\langle u\rangle}{U_{\infty}}\right) \mathrm{d} y
$$


respectively. These two quantities are different measures of the boundary layer thickness in a similar way as the $99 \%$ boundary layer thickness. For the laminar Blasius boundary layer profile the shape factor $H_{12}=2.59$ and $H_{12}=1.38$ for a fully developed turbulent boundary layer flow at high $R e$ (Purtell et al., 1981).

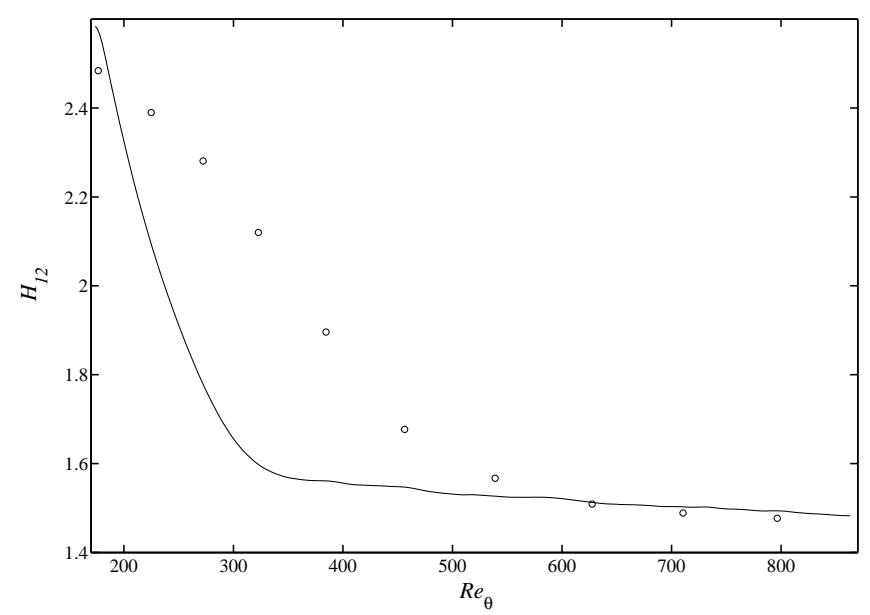

Figure 4.4: Variation of the shape factor with the downstream positions. - Present DNS, ○ Roach and Brierley (1992).

In Figure 4.4, the variation of $H_{12}$ with downstream positions is shown as well as the experimental results from Roach and Brierley (1992). A slowly decrease of $H_{12}$ with the increase of Reynolds number in the turbulent region is observed. The agreement with the experimental data is only good in the fully turbulent region since the experiment was set up to examine the bypass transition in a boundary layer under the influence of free-stream turbulence.

In addition, the profiles of $99 \%$ boundary layer thickness as well as the displacement thickness $\delta_{1}$ and the momentum thickness $\delta_{2}$ are shown in Figure 4.5.
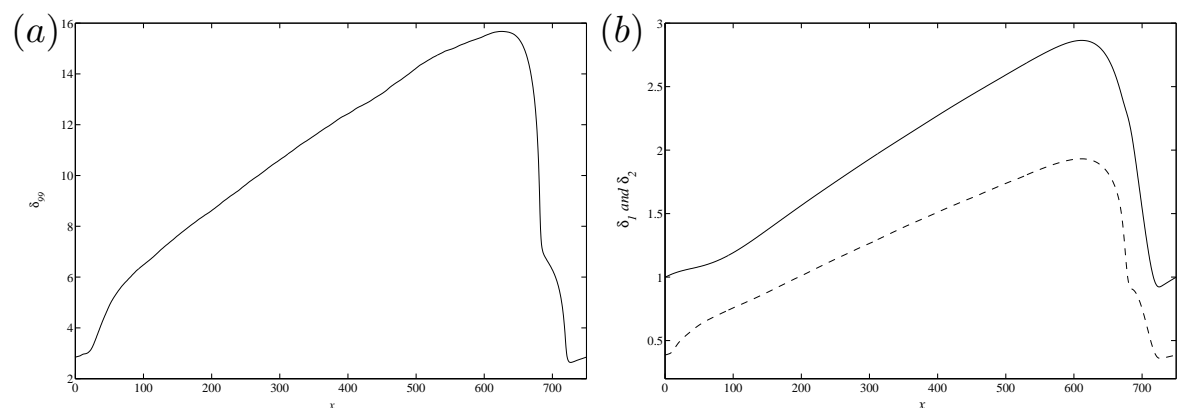

Figure 4.5: Different measures of the boundary layer thickness versus the downstream positions. (a) $99 \%$ boundary layer thickness $\delta_{99}$, (b)—_displacement thickness $\delta_{1},---$ momentum thickness $\delta_{2}$. 
The evolution of the skin friction coefficient $c_{f}$ is shown in Figure 4.6. The computed skin friction shows laminar, transient and turbulent behaviours. An overshoot of the peak is observed which was also reported by previous studies, see e.g. Rai and Moin (1993) or Gilbert and Kleiser (1991). The overshoot has been observed in the coarse resolution simulation of the current case, but slightly diminished as the resolution was increased, which is consistent with the general behaviour of spectral methods applied to transitional flows.

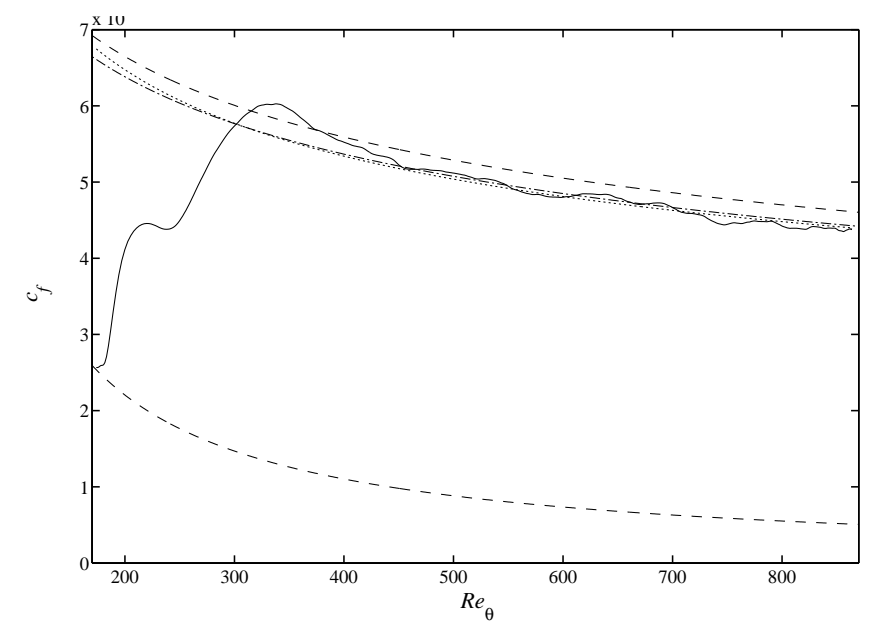

Figure 4.6: Skin friction coefficient $c_{f}$ versus the Reynolds number. — Present DNS, - - - Kays and Crawford (1993), …... Schoenherr (1932), -.-Smits et al. (1983).

In Figure 4.6, the lower dashed line is the theoretical laminar skin friction solution. The upper dashed line is the turbulent solution given by Kays and Crawford (1993) which is

$$
c_{f}=0.025 R e_{\theta}^{-\frac{1}{4}} .
$$

Empirical formulas from Schoenherr (1932) and Smits et al. (1983), which read

$$
c_{f}=0.31\left(\ln ^{2}\left(2 R e_{\theta}\right)+2 \ln \left(2 R e_{\theta}\right)\right)^{-1}
$$

and

$$
c_{f}=0.024 R e_{\theta}^{-\frac{1}{4}},
$$

are also included for comparison. All the solutions collapse with our DNS result very well except the power-law approximation suggested by Kays and Crawford (1993) which is usually valid for high Reynolds number turbulent boundary layer flow, therefore predicts a higher $c_{f}$ than the present case.

The free-stream velocity $U_{\infty}$ and a parameter $\beta$ related to the pressure gradient defined as

$$
\beta=\frac{\delta^{*}}{\tau_{w}} \frac{d p}{d x},
$$


where $\delta^{*}$ is the displacement thickness and $\tau_{w}$ is the shear stress at the wall, are shown in Figure 4.7. These quantities indicate some general features of the flow, i.e. constant free-stream velocity and zero-pressure gradient. The sharp negative peak in the end of the domain of $\beta$ is due to the fringe region.
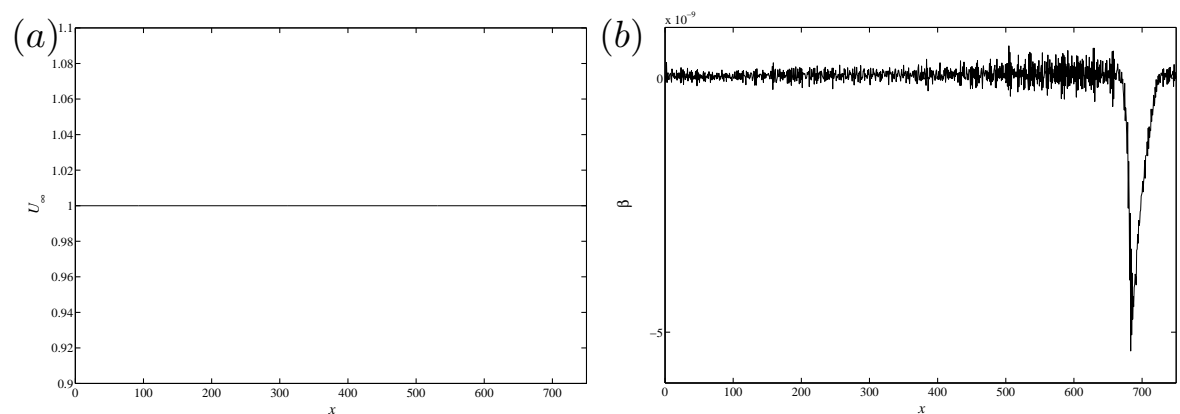

Figure 4.7: Variation of the free-stream velocity $U_{\infty}$ and $\beta$ with downstream positions. (a) Free-stream velocity $U_{\infty},(b) \beta$.

\subsubsection{Turbulent statistics}

The root-mean-square (RMS) values of the velocity and pressure fluctuations and Reynolds shear stress normalised by the friction velocity $u_{\tau}$ are shown in Figure 4.8. The wall-normal distance $y$ is normalised by the viscous scale $\frac{\nu}{u_{\tau}}$. The peak RMS values of all the three components of the velocity, pressure fluctuations and Reynolds shear stress increase with a higher Reynolds number. In particular, the increase for the wall-normal and the spanwise components, $v_{r m s}$ and $w_{r m s}$, are more significant compared to that of the streamwise component $u_{r m s}$. This is in agreement with the previous study by Moser et al. (1999). The peak value of the Reynolds shear stress $-\left\langle u^{\prime} v^{\prime}\right\rangle$ also increases and the peak position moves away from the wall with the increase of the Reynolds number. Apparently, the RMS of the velocity fluctuations are more sensitive to the Reynolds number than the mean quantities. In fact, according to Moser et al. (1999), even the wall limiting behaviour of the RMS profiles vary with different Reynolds number. This is clearly shown in the case of the $p_{r m s}$ in Figure $4.8(e)$. The present hight of the box in viscous units is about 900, so there is not upper boundary influence.

The RMS values of the velocity and pressure fluctuations and Reynolds shear stress normalised by the free-stream velocity $U_{\infty}$ are shown in Figure 4.9. The wall-normal distance $y$ is normalised by the outer scale $\delta^{*}$. Opposite behaviours compared to Figure 4.8 are observed. The peak values of the RMS of the velocity components and the shear stress decrease with a higher Reynolds number as well as the peak positions move towards the wall. In the 

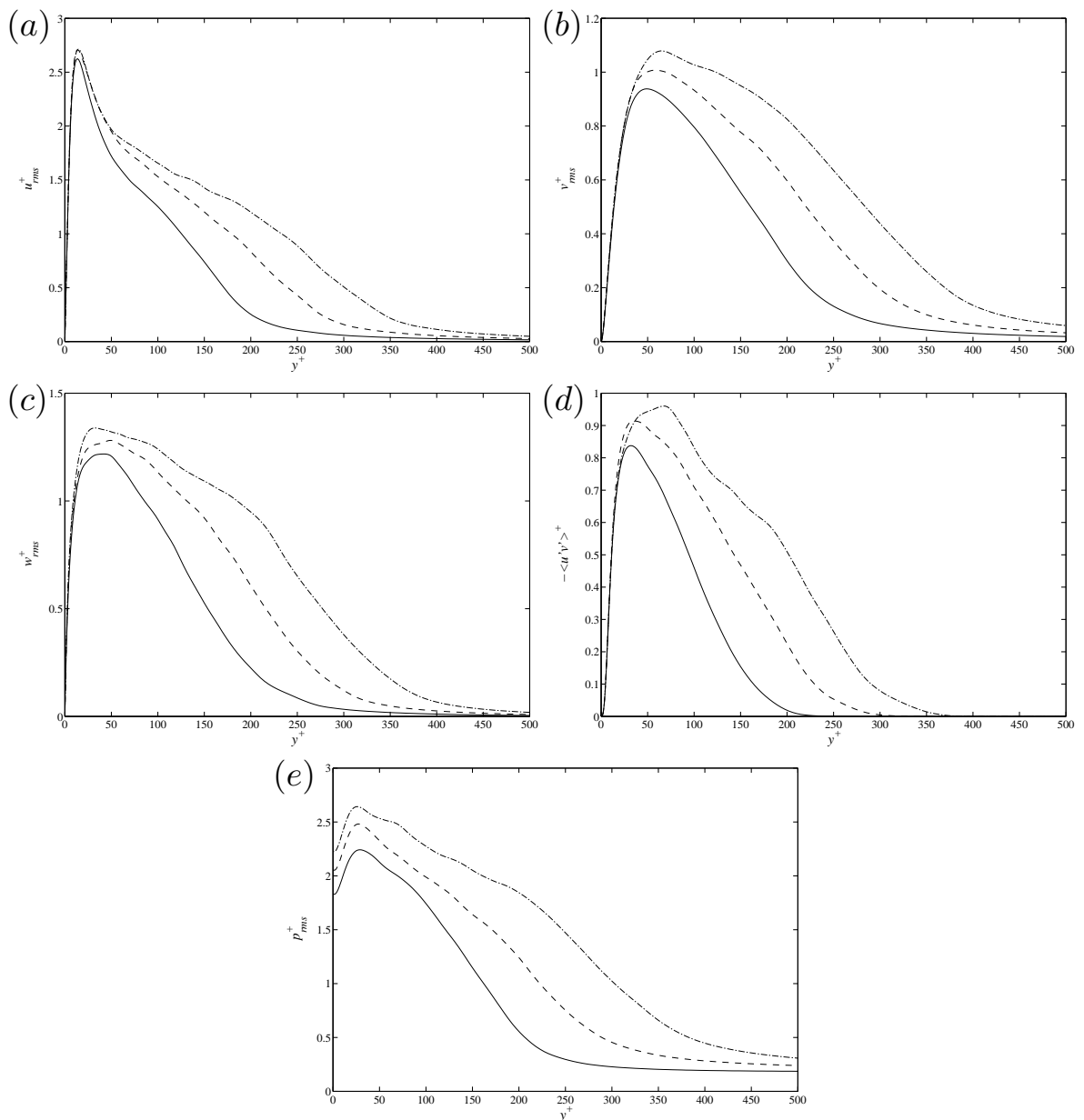

Figure 4.8: RMS values of the velocity, pressure fluctuations and Reynolds shear stress versus downstream positions in inner scaling. —at $R e_{\theta}=$ $396,---$ at $R e_{\theta}=628,-\cdot-$ at $R_{e}=830$. (a) $u_{r m s}$, (b) $v_{r m s},(c) w_{r m s},(d)$ $-\left\langle u^{\prime} v^{\prime}\right\rangle,(e) p_{r m s}$.

outer region, for each quantity, all the profiles at different Reynolds number collapse with each other.

\subsubsection{Vorticity fluctuations}

Figure 4.10 shows the profiles of the non-dimensional RMS values of the vorticity fluctuations, i.e. normalised by the viscous scale $\frac{u_{\tau}^{2}}{\nu}$. The streamwise component has its maximum at the wall and in addition, displays a local maximum at $y^{+} \approx 20$. Almost at the same location, the peak value of the wall-normal component is located. However, the spanwise component of the RMS value of the vorticity fluctuation decreases monotonically from the wall. It is also noted that although the different components behave 

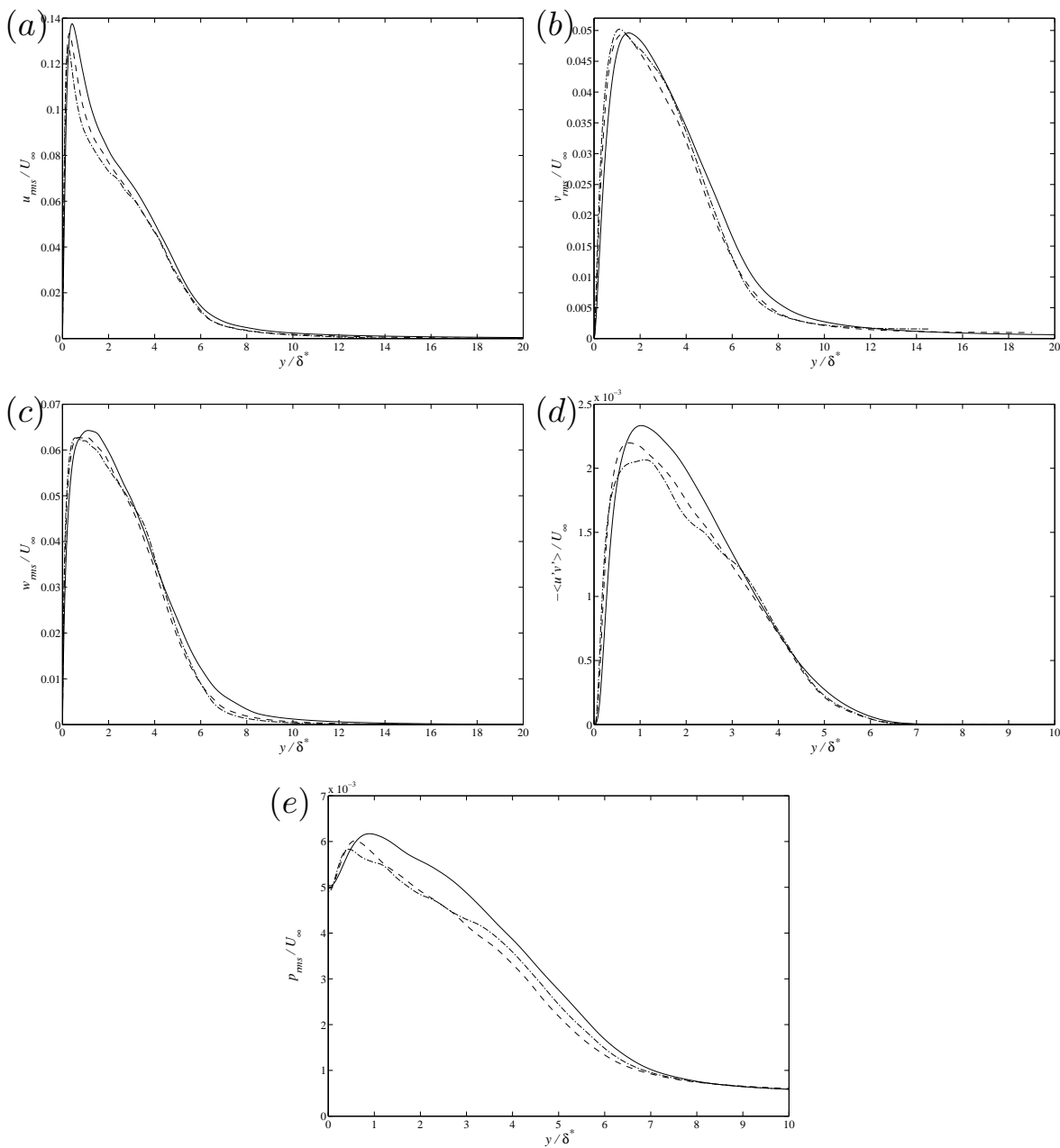

Figure 4.9: RMS values of the velocity, pressure fluctuations and Reynolds shear stress versus downstream positions in outer scaling. — at $R e_{\theta}=$ $396,--$ at $R e_{\theta}=628,-$ - at $\operatorname{Re}_{\theta}=830$. (a) $u_{r m s}$, (b) $v_{r m s},(c) w_{r m s},(d)$ $-\left\langle u^{\prime} v^{\prime}\right\rangle,(e) p_{r m s}$.

differently in the near wall region, away from the wall, i.e. $y^{+}>40$, they are almost identical. This is in contrast to the RMS values of the velocity fluctuations shown in Figure 4.8. According to Moin and Kim (1982), the different behaviours lie in that the relative contribution of small scales to vorticity fluctuations is significantly larger than their contribution to velocity fluctuations and away from the wall the small scales tend to be isotropic.

Figure 4.11 shows the dependence of the RMS values of the vorticity fluctuations on the Reynolds number. In the near wall region, the RMS values of the streamwise and spanwise vorticity fluctuations decrease with the increase of the Reynolds number. The wall-normal vorticity fluctuation, however, tends to become independent of the Reynolds number in the near 


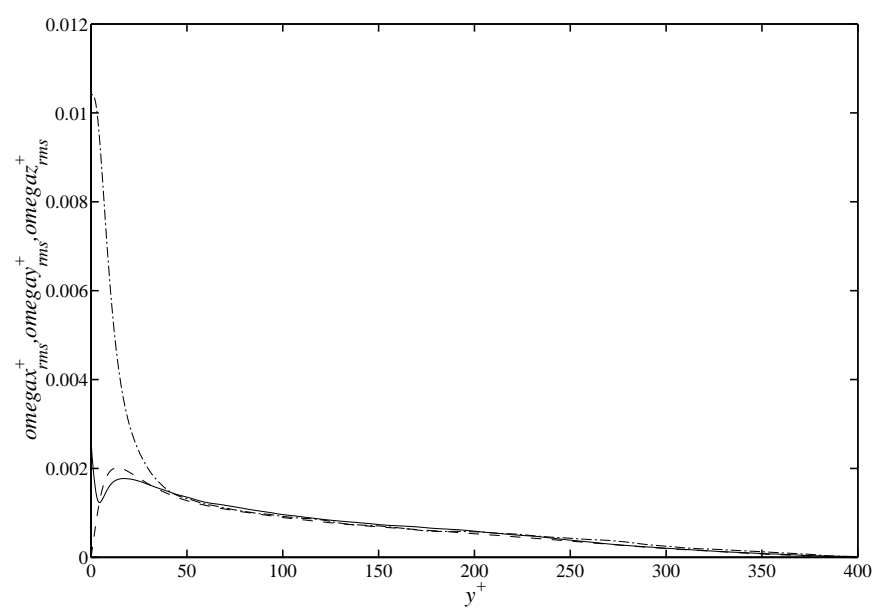

Figure 4.10: RMS values of the vorticity fluctuations at $R e_{\theta}=830$. — Streamwise component, - --wall-normal component, ---Spanwise component.

wall region as reported by Antonia and Kim (1994). The invariance of the wall-normal component $\omega_{y}^{\prime+}$ with Reynolds number is consistent with the invariance of the streak spacing in wall units.

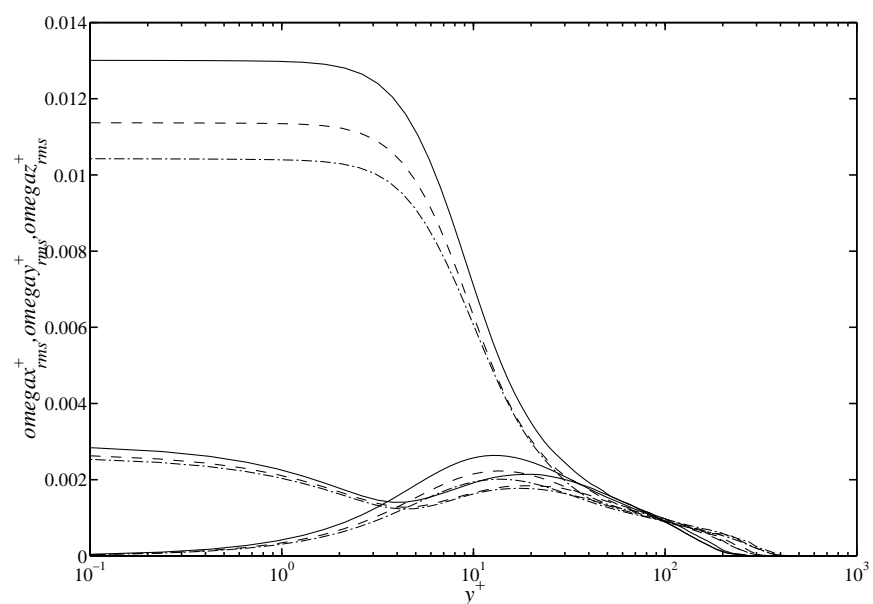

Figure 4.11: RMS values of the vorticity fluctuations with varying Reynolds number. - at $R e_{\theta}=396,---$ at $R e_{\theta}=628,-$ - at $R e_{\theta}=830$.

\subsubsection{Reynolds stress budget}

One main advantage of DNS is to compute the various terms in the budgets of the turbulent transport equations that are difficult to measure. The budget data obtained by DNS gives detailed information to evaluate the turbulence models and is of great help to develop new turbulence modelling 
approaches (Mansour et al., 1988).

The Reynolds stress equation written in a concise way is

$$
\frac{\bar{D} R_{i j}}{\bar{D} t} \equiv \frac{\partial R_{i j}}{\partial t}+\left\langle u_{l}\right\rangle \frac{\partial R_{i j}}{\partial x_{l}}=\mathcal{P}_{i j}-\varepsilon_{i j}+C_{i j}+D_{i j}+T_{i j},
$$

where $R_{i j}$ is the Reynolds stress tensor, $\mathcal{P}_{i j}$ is the production term, $\varepsilon_{i j}$ is the viscous dissipation rate tensor, $C_{i j}$ is the velocity pressure-gradient correlation term and can be split into two terms, namely $\Pi_{i j}$ the pressure strain term and $G_{i j}$ the pressure diffusion term. $T_{i j}$ is the turbulent diffusion term and $D_{i j}$ is the molecular diffusion term. These terms are defined by equation (2.10). Note that in the present study, all the terms appearing in the budgets are explicitly evaluated. The residuals of the individual budgets are of the order $10^{-3}$ to $10^{-2}$ in viscous scaling.
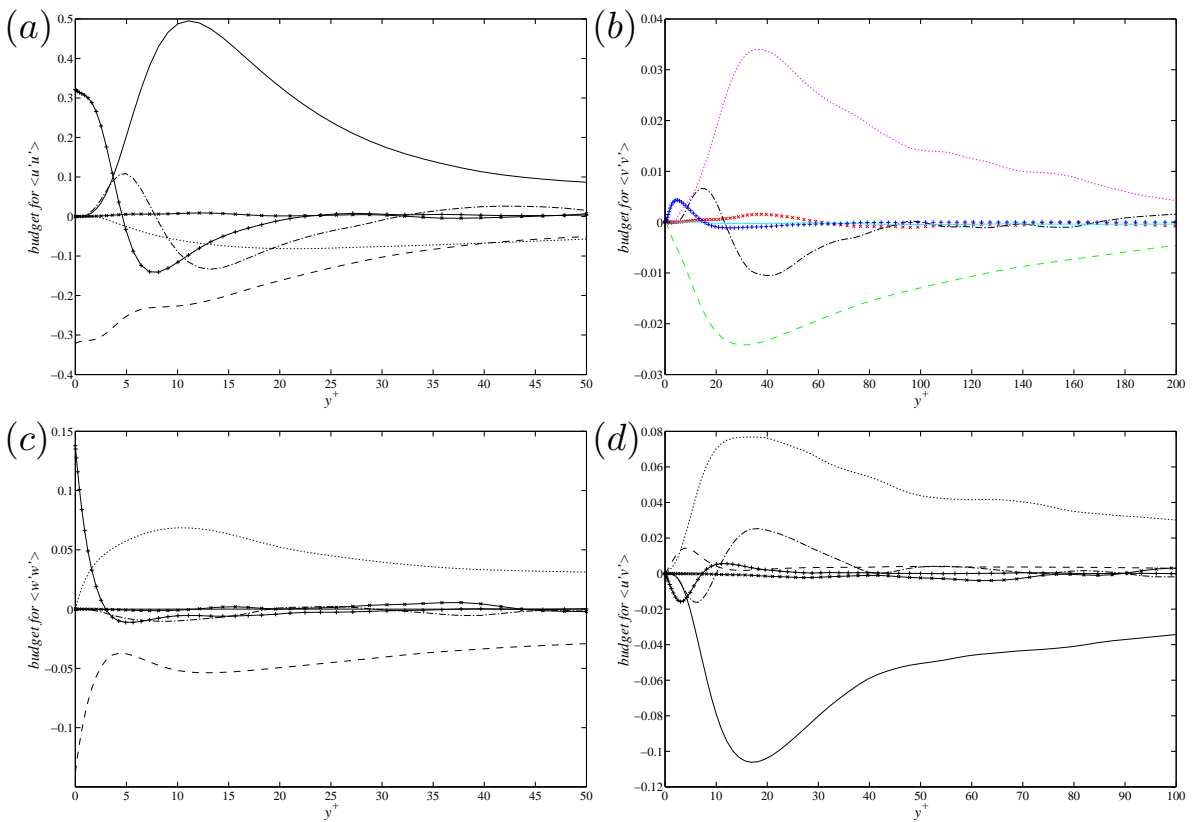

Figure 4.12: Reynolds stress budgets. - Production, - - -Dissipation, -.-Turbulent diffusion, ........ Velocity pressure diffusion, + Molecular diffusion, * Mean convection. (a) Streamwise Reynolds stress $R_{11}$, (b) wallnormal Reynolds stress $R_{22}$, (c) Spanwise Reynolds stress $R_{33},(d)$ Reynolds shear stress $R_{12}$.

Figure 4.12 shows the various budget terms of the Reynolds stresses as functions of the wall-normal distance $y^{+}=\frac{y u_{\tau}}{\nu}$, where $u_{\tau}$ is the friction velocity, $\nu$ is the kinematic viscosity. All the quantities are in wall units, i.e. non-dimensionalized by $\frac{u_{\tau}^{4}}{\nu}$. The budgets for the Reynolds stresses are essentially the same as in Spalart (1988) for a zero-pressure gradient (ZPG) boundary layer. Note that, as opposed to Spalart (1988), in the present 
DNS a spatially developing boundary layer is considered, i.e. the mean convection terms are non-zero. In most of the budget plots, the production and dissipation terms are the dominant terms. The contribution of the mean convection term is negligible near the wall and only becomes noticeable in the outer region of the boundary layer, i.e. $y>0.5 \delta_{99}$. Conversely, the viscous diffusion term carries sufficient energy to balance the viscous dissipation in the immediate neighbourhood of the wall where the production is small. As expected, the near wall behaviour is similar to the shape and magnitude that observed in a channel flow by Moin and Kim (1982) and Couette flow by Komminaho and Skote (2002).

The streamwise Reynolds stress $\left\langle u^{\prime} u^{\prime}\right\rangle$ budget plotted in Figure $4.12(a)$ shows that the production term $\mathcal{P}_{11}$ is the dominant positive term in the range $y^{+}>5$, and has a maximum of 0.5 in the buffer region at $y^{+}=11$, then falls to 0.1 at $y^{+}=50$. The location of the peak production were also found experimentally to be $y^{+} \approx 11$ in channel and pipe flow by Sahay and Sreenivasan (1999). Moreover, the production almost balances with the dissipation and the velocity pressure-gradient correlation terms. For the wall-normal Reynolds stress $\left\langle v^{\prime} v^{\prime}\right\rangle$ and spanwise Reynolds stress $\left\langle w^{\prime} w^{\prime}\right\rangle$ budgets which are shown in Figure $4.12(b)$ and $(c)$, respectively, the velocity pressure-gradient correlation and dissipation terms are dominant rather than the production terms. For the Reynolds shear stress $\left\langle u^{\prime} v^{\prime}\right\rangle$ budget shown in Figure $4.12(d)$, the production and the velocity pressure-gradient correlation terms are important. The other two shear stress budgets are not shown since the different terms in those budgets are just varying around zero.

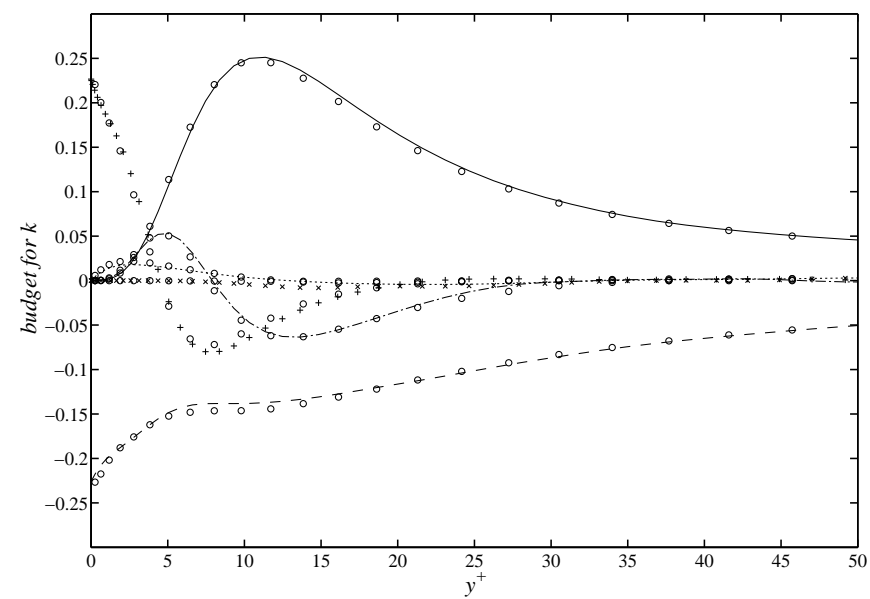

Figure 4.13: Budget for the turbulent kinetic energy $k$. - Production, - - - Dissipation, -- - Turbulent convection, ........ Velocity pressure diffusion, + Viscous diffusion, $*$ Mean convection, o Spalart (1988).

A comparison of the budget for the turbulent kinetic energy $k=\frac{1}{2}\left\langle u_{i}^{\prime} u_{i}^{\prime}\right\rangle$ with the DNS data from Spalart (1988) is shown in Figure 4.13. The 
Reynolds number based on the momentum thickness for both cases is 670 . From the plots we see that the agreement between both the DNS data is very good.

An investigation of the dependence on the Reynolds number of $R_{i i}$ (no summation) and $R_{12}$ is plotted in Figure 4.14. All the components increase with higher Reynolds number. Especially, for the streamwise component $R_{11}$, the wall values of the dissipation and molecular diffusion increase appreciably towards large Reynolds number.
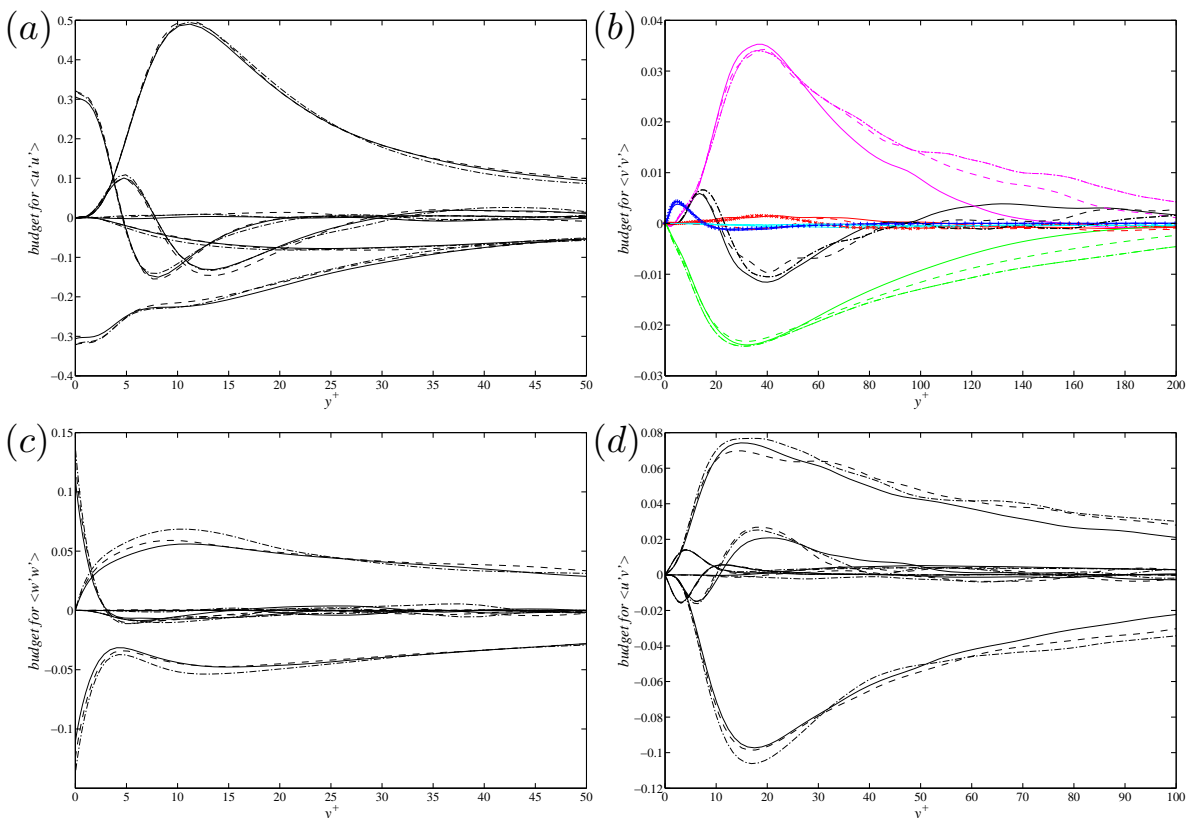

Figure 4.14: Reynolds stress budgets compared at different Reynolds numbers. - at $R e_{\theta}=396,--$ at $R e_{\theta}=628,-\cdot-$ at $R e_{\theta}=830$. (a) Streamwise component $R_{11}$, (b) Wall-normal component $R_{22}$, (c) Spanwise component $R_{33},(d)$ Shear component $R_{12}$.

\section{Pressure strain correlation}

The pressure strain terms $\Pi_{i j}$ and pressure diffusion term $G_{i j}$ are actually split from the velocity pressure-gradient term $C_{i j}$

$$
C_{i j}=\Pi_{i j}+G_{i j} .
$$

It is well known that the pressure strain terms $\Pi_{i j}$ play a significant role in the energy redistribution. The profiles of the diagonal elements of $\Pi_{i j}$ as well as an enlargement in the near wall region are shown in Figure 4.15. DNS data from Moser et al. (1999) is also included for comparison. These three terms are responsible for the exchange of the energy between the three components of the turbulent kinetic energy (Hinze, 1975). A positive sign of 
$\Pi_{i i}$ (no summation) indicates an energy gain from other components whereas a negative sign denotes an energy loss. Very close to the wall, one can find that almost all the energy is transferred from the wall-normal component $\Pi_{22}$ to the horizontal components $\Pi_{11}$ and $\Pi_{33}$. This phenomenon was observed by Daly and Harlow (1970) and later termed by Moin and Kim (1982) as the splatting or impingement effect. The splatting effect is an important property of the flow in the vicinity of the wall and should be taken into account in the modelling of the near wall turbulence (Moin and Kim, 1982). For more discussions about the split about the pressure velocity gradient terms, see e.g. Mansour et al. (1988).
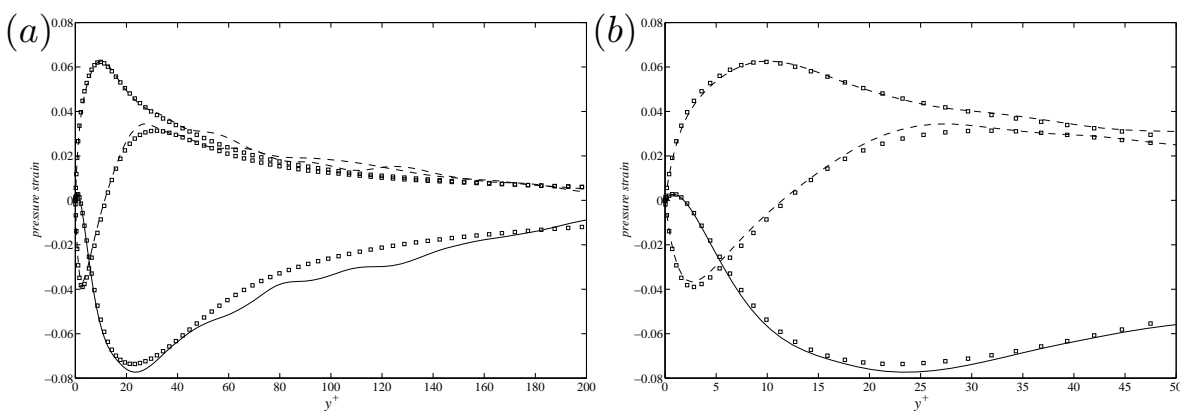

Figure 4.15: Pressure strain correlation terms versus the wall-normal distance at $R e_{\theta}=670$. - Streamwise component $\Pi_{11},---$ Wall-normal component $\Pi_{22},-\mathbf{-}$-Spanwise component $\Pi_{33}, \square$ Moser et al. (1999) at $R e_{\tau}=590$.

\subsubsection{Reynolds stress anisotropy}

The Reynolds stress anisotropy tensor is defined by

$$
a_{i j}=\frac{R_{i j}}{k}-\frac{2}{3} \delta_{i j}
$$

where $R_{i j}$ is the Reynolds stress tensor, $k$ is the turbulent kinetic energy and $\delta_{i j}$ is the Kronecker delta. The Reynolds stress anisotropy tensor $a_{i j}$ has zero trace, i.e. $a_{i i}=0$, and thus two non-zero invariants, $\mathrm{II}_{a}$ and $\mathrm{III}_{a}$. They are defined by

$$
\mathrm{II}_{a}=a_{i j} a_{j i}
$$

and

$$
\operatorname{III}_{a}=a_{i j} a_{j k} a_{k i} .
$$

As described by Lumley and Newman (1977), all anisotropic states can be characterised by these two invariants and represented in the so-called anisotropy invariant map (AIM) which is bounded by the lines $8 / 9+\mathrm{III}_{a}=$ $\mathrm{II}_{a}$ and $6 \mathrm{III}_{a}^{2}=\mathrm{II}_{a}^{3}$. These lines represent two-component and axisymmetric 
turbulence, respectively. Sometimes it is convenient to replace $\mathrm{II}_{a}$ and $\mathrm{III}_{a}$ by another two independent variables $\eta$ and $\zeta$ defined by

$$
6 \eta^{2}=-2 \mathrm{II}_{a}
$$

and

$$
6 \zeta^{3}=3 \mathrm{III}_{a} .
$$

The AIM path for the boundary layer flow of different wall-normal positions at $R e_{\theta}=830$ is shown in Figure 4.16. The present case is very similar to the results of boundary layer flow and Couette flow by Komminaho and Skote (2002). Close to the wall the turbulence is very near the two-component limit, approaching the one-component limit near the edge of the viscous sub-layer at $y^{+} \approx 7$ and then the AIM path turns towards the isotropic state. There is some agglomeration of points at $y^{+} \approx 150$ which indicates the log region and is also observed by Komminaho and Skote (2002).

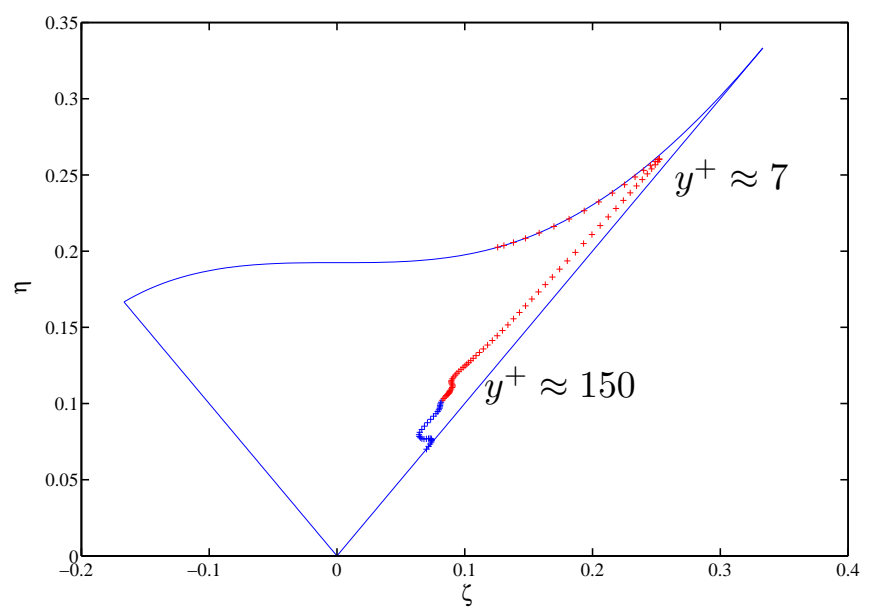

Figure 4.16: $\mathrm{AIM}$ at $R e_{\theta}=830$ versus wall-normal positions.

\subsubsection{Higher order statistics}

The skewness and flatness factors for a statistically stationary variable $a(x, y, z, t)$ are defined as

$$
S(a)=\frac{\left\langle a^{\prime 3}\right\rangle}{\left\langle a^{\prime 2}\right\rangle^{\frac{3}{2}}}
$$

and

$$
F(a)=\frac{\left\langle a^{\prime 4}\right\rangle}{\left\langle a^{\prime 2}\right\rangle^{2}} .
$$

The skewness indicates the relative extent of negative and positive fluctuations about the mean value. If $S(a)>0$, the positive fluctuations dominate 
while the negative fluctuations dominate if $S(a)<0$. The flatness determines the extent of symmetric but remote deviations from the mean. For a quantity which has a Gaussian distribution, the skewness and flatness are 0 and 3, respectively. The velocity and pressure skewness and flatness factors in the near wall region compared with the simulation by Moser et al. (1999) are plotted in Figures 4.17 and 4.18. As seen from Figure 4.17,
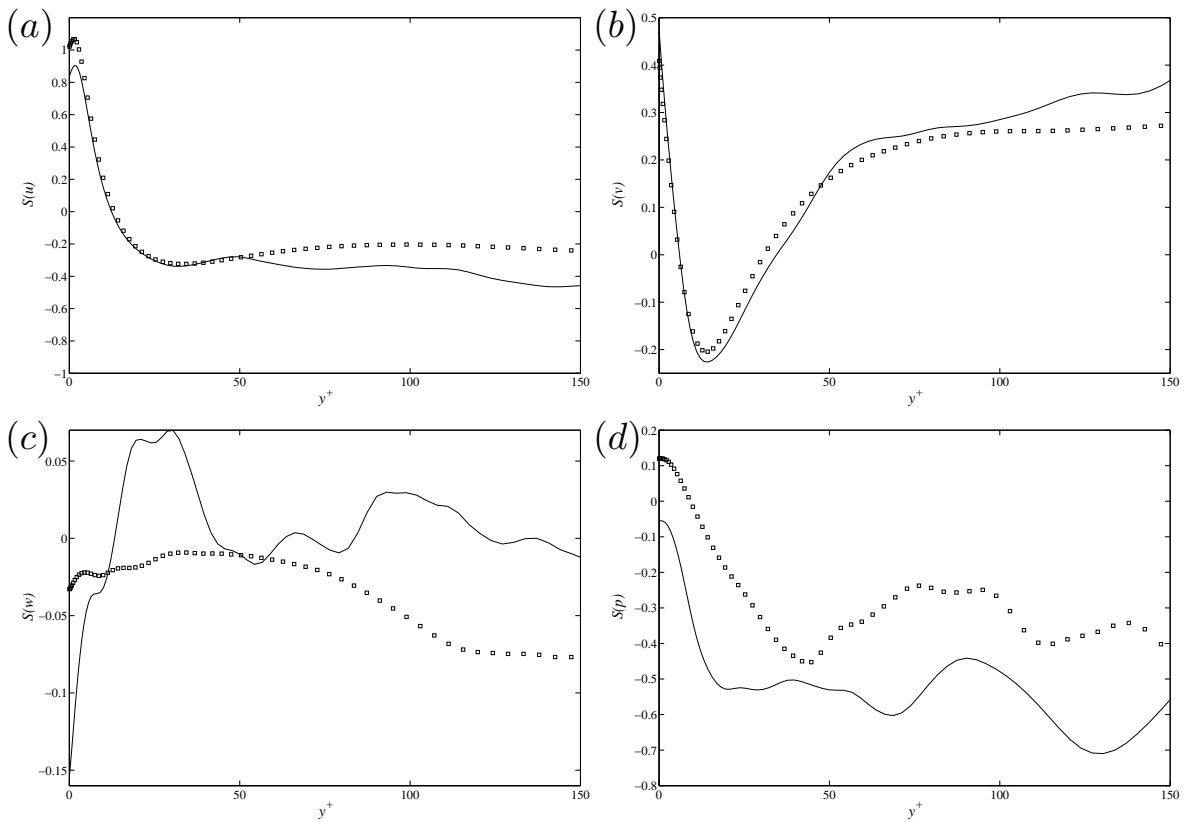

Figure 4.17: Skewness factor distributions of the velocities and pressure at $R e_{\theta}=830$. - DNS, $\square$ Moser et al. (1999) at $R e_{\tau}=590$. (a) $S(u)$, (b) $S(v),(c) S(w),(d) S(p)$.

all the skewness factor distributions except $S(w)$ and $S(p)$ are in very good agreement in the near wall region with Moser et al. (1999). Moreover, the present DNS predicts lower values at the wall than those from Moser et al. (1999) for the computed flatness of all the velocity components. Especially, for the flatness of the wall-normal velocity, the present DNS result is about 15, while the one from Moser et al. (1999) is about 40. For the flatness of the wall-normal velocity component see also the discussion in Durst and Beronov (2003). For the flatness factor of the pressure fluctuation at the wall, Kim (1989) reported 5.0, Schewe (1983) reported 4.9 while the present DNS is 4.7 .

The velocity and pressure skewness and flatness factors as a function of the wall-normal distance are shown in Figure 4.19. The skewness and flatness factors of a Gaussian distribution are also included for reference. All the skewness factors deviate from those values of a Gaussian distribution except the spanwise velocity component. For the flatness factor distributions, all the velocity components and pressure reach their maxima at the wall which 

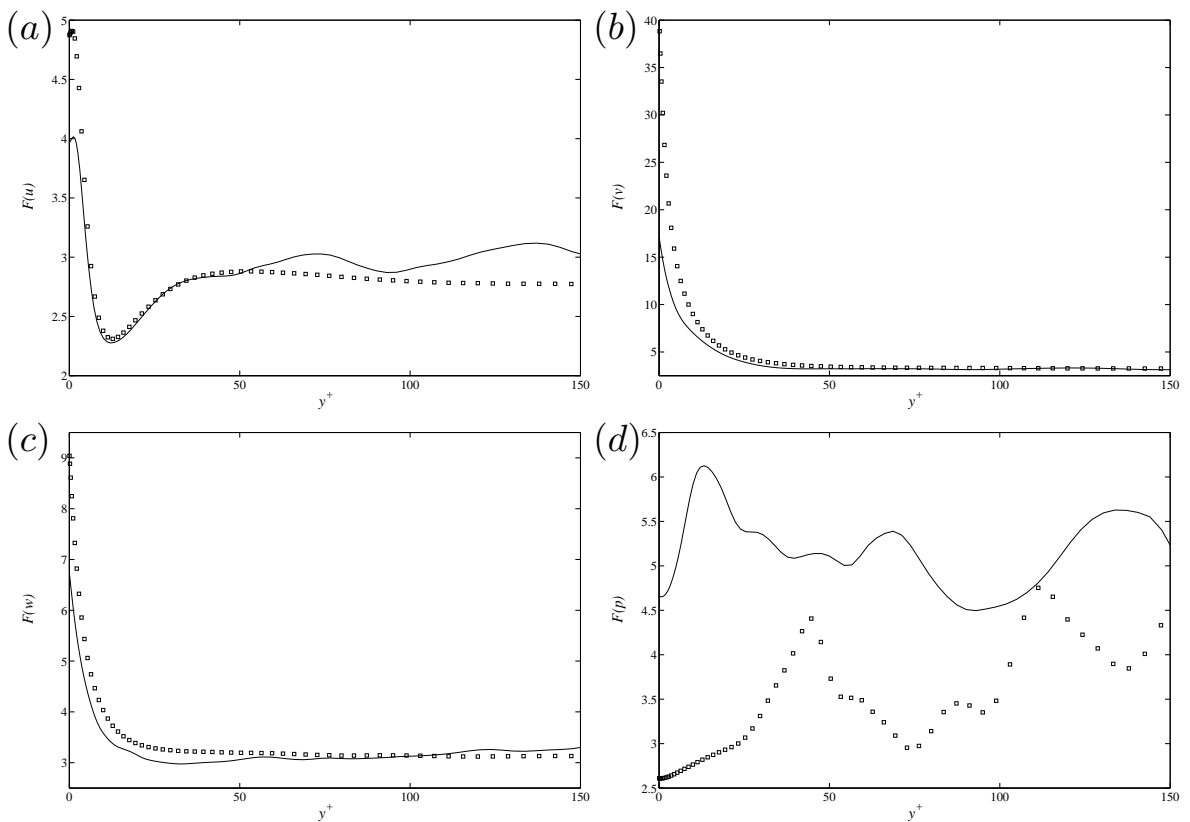

Figure 4.18: Flatness factor distributions of the velocities and pressure at $R e_{\theta}=830$. - DNS, $\square$ Moser et al. (1999) at $R e_{\tau}=590$. (a) $F(u),(b)$ $F(v),(c) F(w),(d) F(p)$.
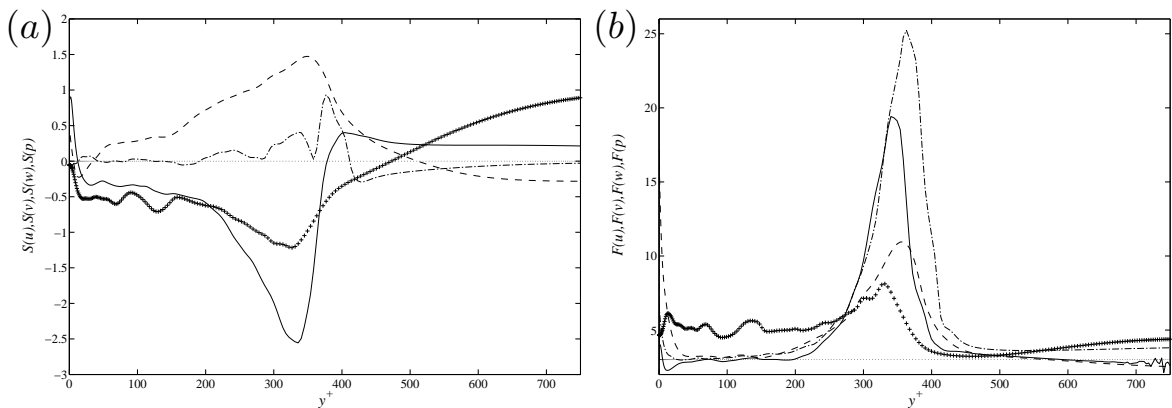

Figure 4.19: Skewness and flatness factor distributions of the velocities and pressure at $R e_{\theta}=830$. - Streamwise velocity, - - - wall-normal velocity, -.-Spanwise velocity, + Pressure, ….... Gaussian distribution. (a) Skewness factor, $(b)$ Flatness factor.

means the turbulence in the vicinity of the wall is highly intermittent. Away from the wall, the pressure flatness is significantly higher than those of the velocity fluctuations which implies in the outer region of the boundary layer the pressure is more intermittent and can also be shown by the probability density function of these quantities. A similar behaviour of the pressure in the channel flow is reported by Kim et al. (1987).

From Figure 4.19, we also observe that there exist very high peaks near the boundary layer edge at $y^{+} \approx 350$ of the skewness and flatness factors 
for all the velocities and pressure components which are not present in the channel flow simulation. These high peaks are also observed in an experimental study in a turbulent boundary layer flow by Österlund and Johansson (1999). These are clearly related to the structure of the outer boundary layer edge, i.e. intermittency of the wake region. Outside the boundary layer, all the skewness and flatness factors decrease rapidly toward the values of 0 and 3, respectively. This is consistent with the Gaussian character in the free-stream.

\subsubsection{Turbulent structure of the flow fields}

Moin and Kim (1982) investigated the organised structure associated with wall-bounded flows. But due to the coarse mesh used in the computation, some quantitative structure such as the streak spacing in the wall region was not consistent with the experiments. In the present simulation, we have a sufficient grid resolution, $\Delta z^{+} \approx 6.3$, to capture the formation of the near wall streak.

The spanwise two-point correlation coefficient of a statistically stationary variable $a(x, y, z, t)$ is defined by

$$
R_{a a}(x, y, \Delta z, t)=\frac{\left\langle a^{\prime}(x, y, z, t) a^{\prime}(x, y, z+\Delta z, t)\right\rangle}{\left\langle a^{\prime}(x, y, z, t)^{2}\right\rangle},
$$

where $\Delta z$ is the spanwise separation. The spanwise two-point correlations for the three velocity components and pressure at $y^{+}=4.9$ are plotted in Figure 4.20. For each of the velocity correlations, a minimum is observed. The streamwise correlation $R_{u u}$ first becomes negative and reaches a minimum at $\Delta z^{+} \approx 60$. The magnitude of this minimum first increases with increasing wall-normal distance, and then reaches a maximum at the wallnormal position where the $u_{r m s}$ has a maximum. Afterwards, it will decrease until it disappears in the free-stream (not shown here). The separation at which the minimum occurs is an estimate of the distance between a high speed streak and a low speed streak, so the mean spacing of streaks should be roughly twice this distance. The presence of the minimum of $R_{v v}$ at $\Delta z^{+} \approx 25$ is consistent with the numerical results of Kim et al. (1987). This separation is a measure of the mean diameter of the streamwise vortices in the near wall region. The minimum of $R_{w w}$ appears at $\Delta z^{+} \approx 60$ and indicates the existence of the counter-rotating vortex pairs. According to Kim et al. (1987), the minimum of $R_{w w}$ does not exist for $y^{+}>30$ and is more likely due to the splatting effect which is also true for the present DNS. One interesting thing is that the spanwise two-point correlation coefficient of the pressure does not have the negative excursion which is also observed by Kim (1989).

Figure 4.21 shows the dependence of the spanwise two-point correlation coefficients of the streamwise velocity fluctuation on various downstream 


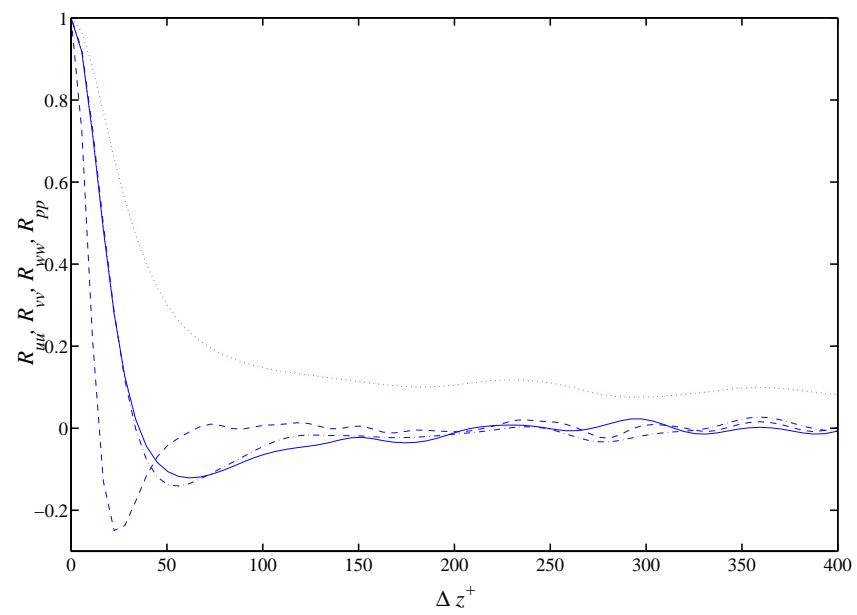

Figure 4.20: Spanwise two-point correlation coefficients of the velocity and pressure fluctuations at $y^{+}=4.9$ with $R e_{\theta}=830$. $-R_{u u},---R_{v v}$, $-\cdot R_{w w}, \cdots \cdots \cdot R_{p p}$.

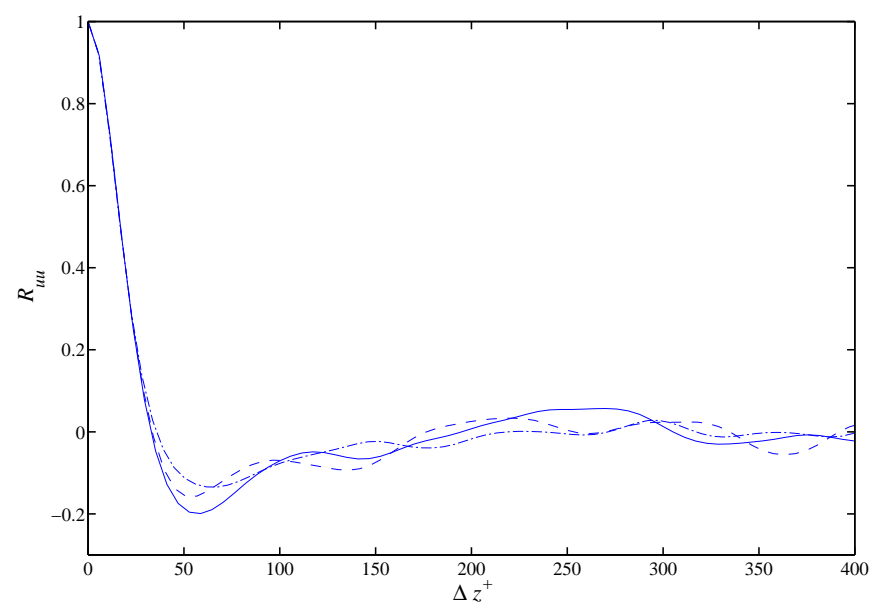

Figure 4.21: Spanwise two-point correlation coefficients of the streamwise velocity fluctuation with varying Reynolds numbers. - at $R e_{\theta}=628$, - - - at $\operatorname{Re}_{\theta}=728,-$ - at $R e_{\theta}=830$.

positions. It is interesting to note that the first minimum becomes less prominent as the Reynolds number increases. This is due to the fact that the streamwise streaks are clustered for a higher Reynolds number as reported by Abe et al. (2001). We also found that the mean streak spacing was increasing as departing from the wall.

The present low-speed streak spacing shown in Figure 4.22 predicts a larger value than those experimental data of turbulent boundary layer flow by Smith and Metzler (1983), Westin et al. (1994) and DNS data of turbulent channel flow by Kim et al. (1987). This can possibly be explained by the 


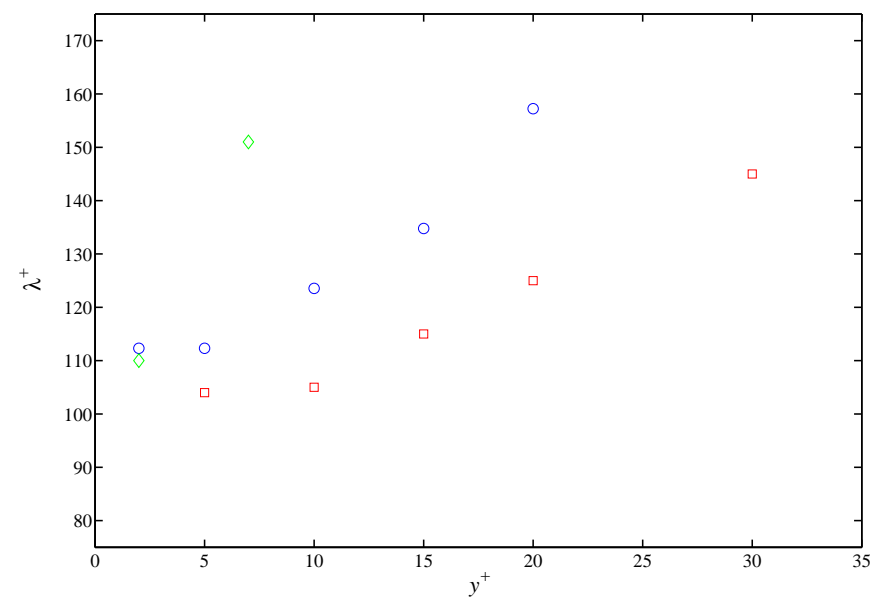

Figure 4.22: Variation of the mean spanwise steak spacings with varying wall-normal positions. $\circ$ Present DNS, $\square$ Smith and Metzler (1983), $\diamond$ Gupta et al. (1971).

present choice of the spanwise domain size which is a little bit small, i.e. about $z^{+} \approx 750$.
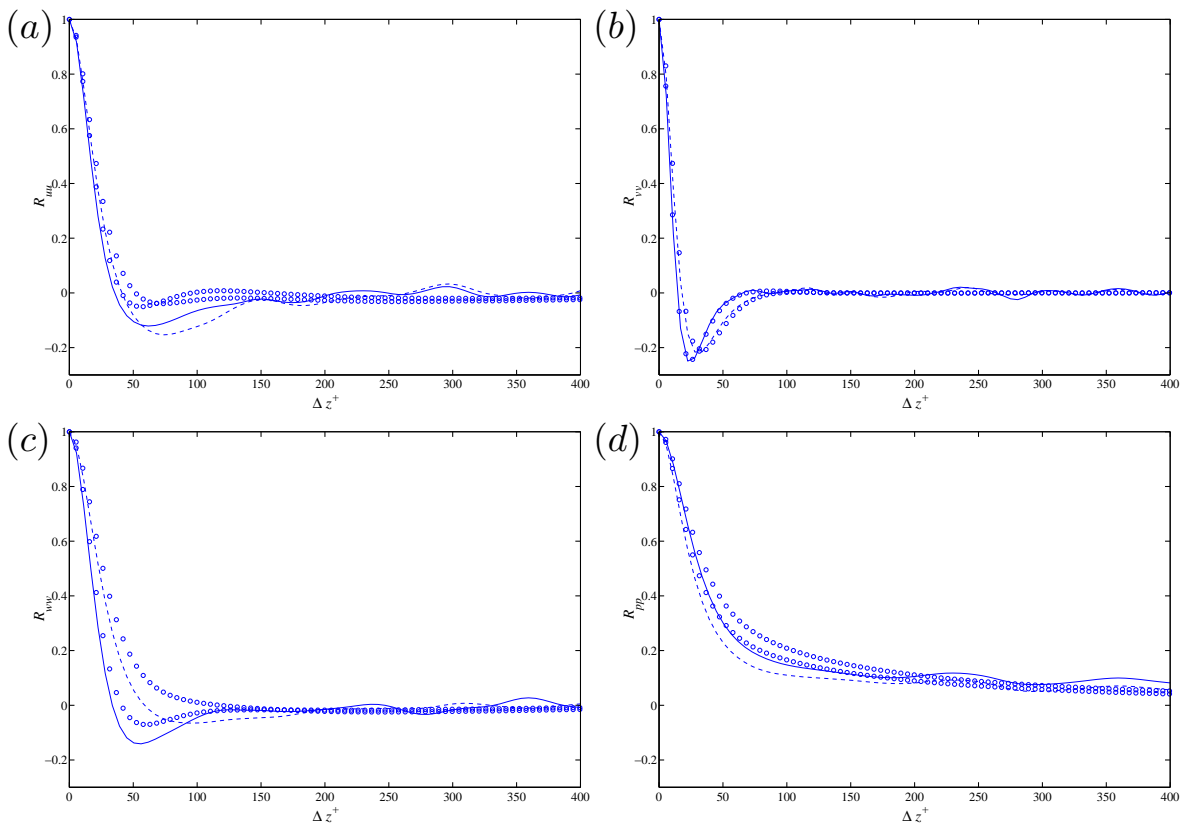

Figure 4.23: Comparison of the spanwise two-point correlation coefficients of the velocity and pressure fluctuations with other simulation. - at $y^{+}=4.9$ with $R e_{\theta}=830,---$ at $y^{+}=14.5$ with $R e_{\theta}=830$, ○ Iwamoto (2002) at $R e_{\tau}=645$. (a) $R_{u u}$, (b) $R_{v v}$, (c) $R_{w w}$, (d) $R_{p p}$.

A comparison with the DNS data from Iwamoto (2002) with $R e_{\tau}=645$ 
at $y^{+} \approx 5$ and 15 is displayed in Figure 4.23. Due to the difference between the Reynolds numbers, the first minimum of $R_{u u}$ and $R_{w w}$ become less prominent as mentioned earlier. However, one interesting thing is that the wall-normal component $R_{v v}$ seems to be Reynolds number independent.

\subsubsection{Probability density functions}

A different perspective on the characteristics of the fluctuations of one or more variables is provided by the probability density function (PDF). The PDF distributions of the velocity and pressure fluctuations at various wallnormal positions, ranging from $y^{+}=5$ in the viscous sub-layer to $y^{+}=500$ in the free-stream, are shown in Figures 4.24 to 4.27. A Gaussian distribution with zero mean and matching variance is also shown as a reference. The velocity and pressure fluctuations are normalised by the corresponding RMS values, and the probability density distributions are normalised in such a way that the area under each curve is unity.

The PDF of the streamwise velocity fluctuation is positively skewed in the near wall region, which can also been seen from the skewness factor $S(u)$, and successively changes to negatively skewed. Very far away from the wall, e.g. in the free-stream, the PDF of the streamwise velocity fluctuation is close to the Gaussian distribution. The probability density distribution at $y^{+}=4.87$ is highly asymmetric where the skewness factor is 0.7. A long positive tail at this wall-normal position is observed and can be interpreted as being caused by the sweep motion. At $y^{+} \approx 15, P\left(u^{\prime}\right)$ is nearly symmetric with $S(u) \approx 0$. The general shape of $P\left(u^{\prime}\right)$ agrees well with the experiment study by Zarić (1975) at available wall-normal positions. Conversely, the PDF of the wall-normal velocity fluctuation is close to the Gaussian distribution in the near wall region, and depart from the Gaussian distribution far away from the wall which is also reported by Nagano and Tagawa (1988). However, very close to the wall and at the edge of the boundary layer, the large $F\left(v^{\prime}\right)$ values lead to more pointy distributions. The PDF of the pressure fluctuation is negatively skewed throughout the boundary layer until in the free-stream. By comparing these PDF with those of the streamwise velocity fluctuation, we found that relatively large contributions are from the high intensity fluctuations, i.e. $\left|\frac{p}{p_{r m s}}\right|>3$. On the other hand, the corresponding contribution to the streamwise velocity fluctuation is negligible. This is also reported by Kim and Lee (1991) in turbulent channel flow.

\subsubsection{Quadrant analysis}

During the past 50 years, there have been numerous studies of the turbulent flow field near the wall to investigate the structure of the Reynolds stress which associated with the turbulence production. Many of the studies show that the low speed fluid in the region near the wall occasionally erupts 

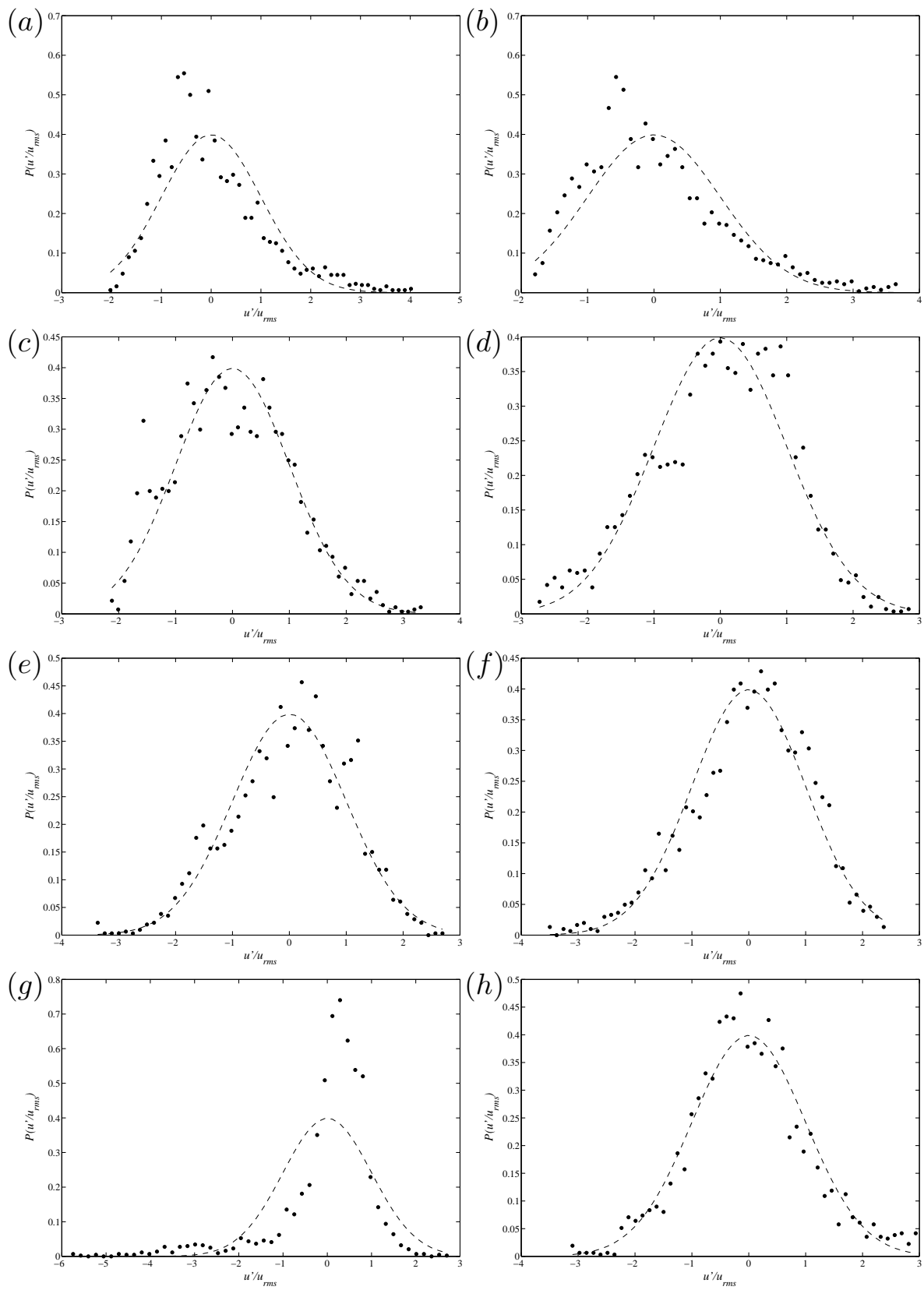

Figure 4.24: PDF of the streamwise velocity fluctuation $u^{\prime}$ at $R e_{\theta}=830$. (a) $y^{+}=1.92, S(u)=0.90,(b) y^{+}=4.87, S(u)=0.67,(c) y^{+}=9.74, S(u)=$ 0.17, (d) $y^{+}=29.3, S(u)=-0.33,(e) y^{+}=48.7, S(u)=-0.28,(f) y^{+}=$ $97.4, S(u)=-0.34,(g) y^{+}=292.4, S(u)=-1.84,(h) y^{+}=486.7, S(u)=$ 0.25 .

violently into the high speed outer region of the boundary layer. Kline et al. (1967) and Kim et al. (1971) were among the first to name this process 

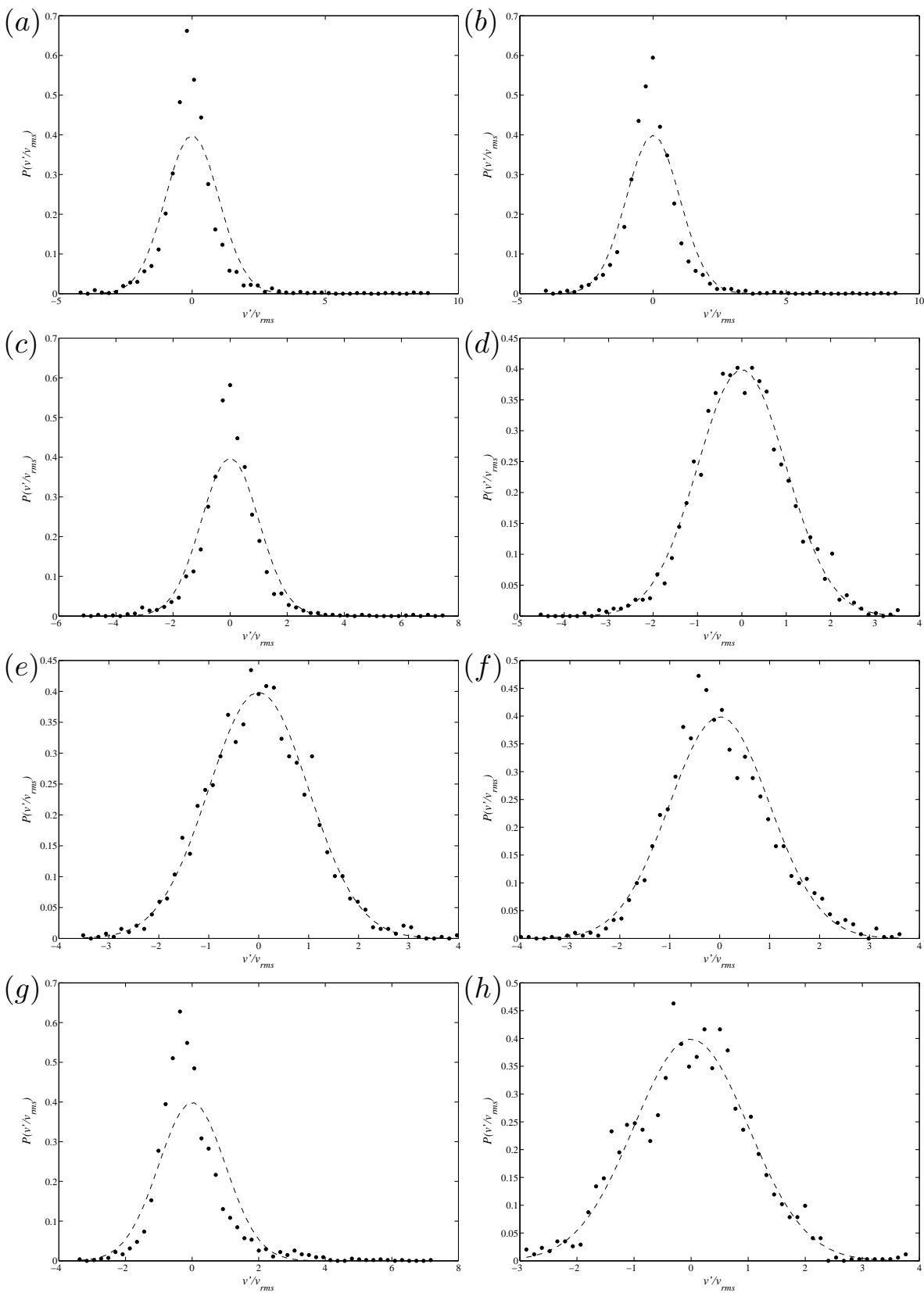

Figure 4.25: PDF of the wall-normal velocity fluctuation $v^{\prime}$ at $R e_{\theta}=830$. (a) $y^{+}=1.92, S(v)=0.30,(b) y^{+}=4.87, S(v)=0.08,(c) y^{+}=9.74, S(v)=$ $-0.17,(d) y^{+}=29.3, S(v)=-0.06,(e) y^{+}=48.7, S(v)=0.16,(f) y^{+}=$ $97.4, S(v)=0.28,(g) y^{+}=292.4, S(v)=1.16,(h) y^{+}=486.7, S(v)=0.09$.

as "bursting" which was later used by Corino and Brodkey (1969), Wallace et al. (1972), Willmarth and Lu (1972) and Lu and Willmarth (1973) among others. During a bursting process, as described by Kim et al. (1971), the low 

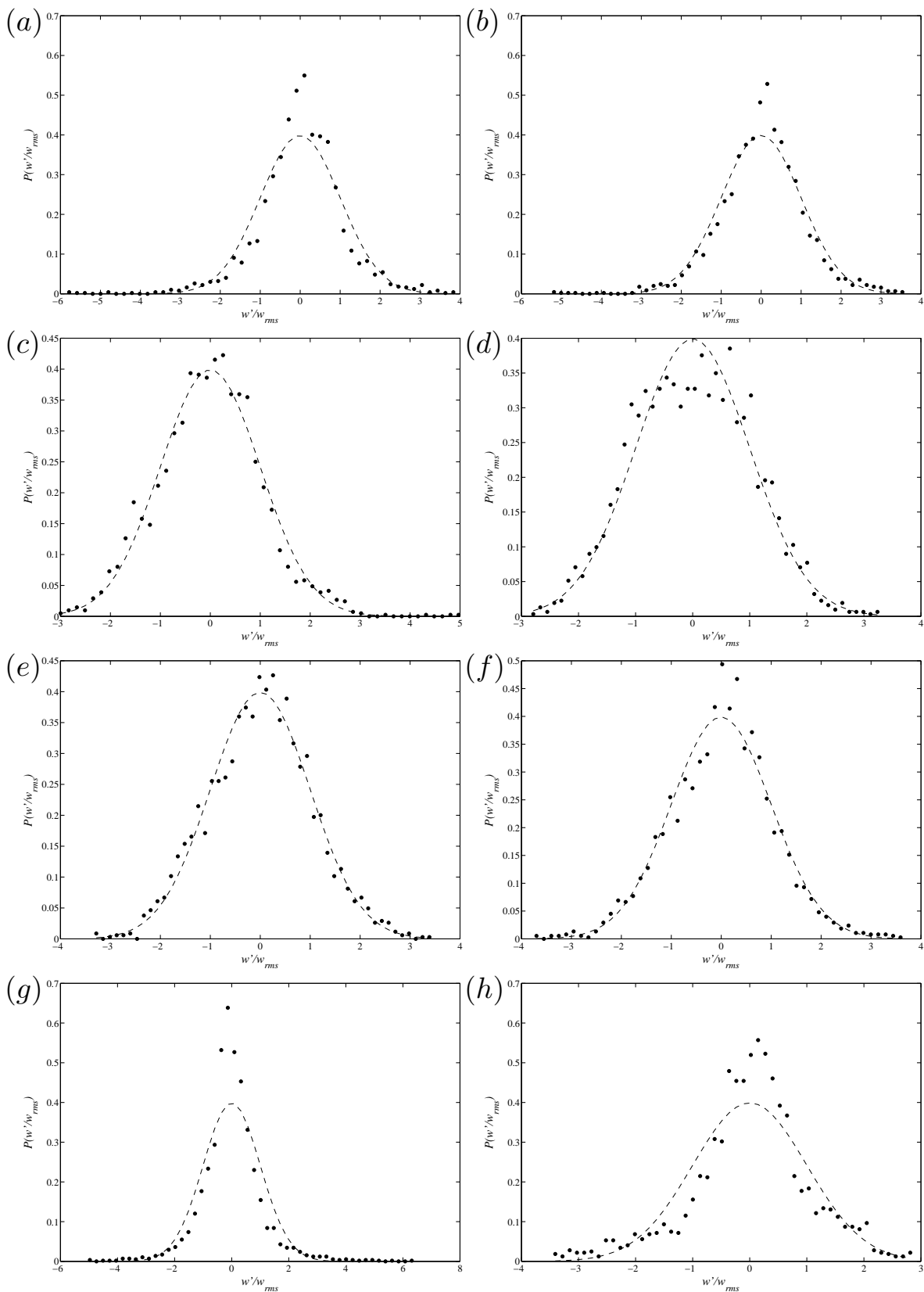

Figure 4.26: PDF of the spanwise velocity fluctuation $w^{\prime}$ at $R e_{\theta}=830$. (a) $y^{+}=1.92, S(w)=-0.10,(b) y^{+}=4.87, S(w)=-0.05$, (c) $y^{+}=$ $9.74, S(w)=-0.03,(d) y^{+}=29.3, S(w)=0.07,(e) y^{+}=48.7, S(w)=$ $-0.01,(f) y^{+}=97.4, S(w)=0.03,(g) y^{+}=292.4, S(w)=0.14,(h) y^{+}=$ 486.7, $S(w)=-0.16$.

speed streaks were observed first to lift up slowly away from the wall, then start a growing oscillation and finally break up into more chaotic motion. 

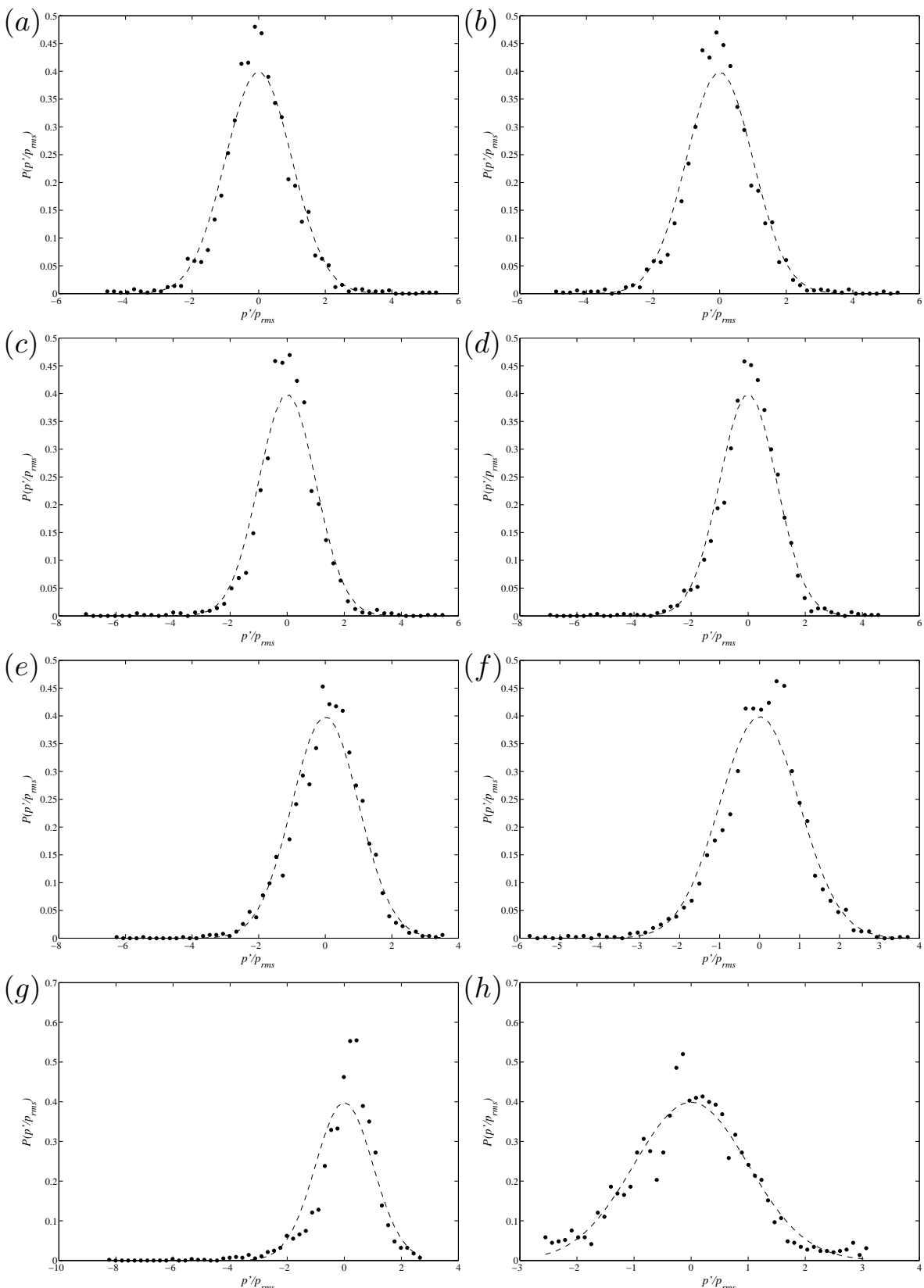

Figure 4.27: PDF of the pressure fluctuation $p^{\prime}$ at $R e_{\theta}=830$. (a) $y^{+}=$ $0, S(p)=0.01,(b) y^{+}=4.87, S(p)=-0.06,(c) y^{+}=9.74, S(p)=-0.26$, (d) $y^{+}=29.3, S(p)=-0.48,(e) y^{+}=48.7, S(p)=-0.51,(f) y^{+}=$ 97.4, $S(p)=-0.48,(g) y^{+}=292.4, S(p)=-1.13$, (h) $y^{+}=486.7, S(p)=$ -0.07 .

Some of the insights into boundary layer structure gained from DNS are summarized in the paper by Robinson (1991). 
The quadrant analysis is one of the various structures detection techniques which provides detailed information about the contributions to the total production from various events occurring in the flow and has been notably used, e.g. by Rajagopalan and Antonia (1982), Alfredsson and Johansson (1984) and Wark and Nagib (1991). The analysis divides the Reynolds shear stress into four categories according to the signs of $u^{\prime}$ and $v^{\prime}$. The first quadrant, $u^{\prime}>0$ and $v^{\prime}>0$, contains outward motion of high speed fluids; the second quadrant, $u^{\prime}<0$ and $v^{\prime}>0$, contains the motion associated with the ejections of the low speed fluids moving from the wall; the third quadrant, $u^{\prime}<0$ and $v^{\prime}<0$, contains wall-ward motion of low speed fluids; the fourth quadrant, $u^{\prime}>0$ and $v^{\prime}<0$, contains the motion associated with the sweep events of the high speed fluids moving towards the wall. Thus the first and third quadrant contribute to the negative production, i.e. positive Reynolds stress, while the second and fourth quadrant contribute to the positive production, i.e. negative Reynolds stress. Table 4.2.9 summarises the signs of the $u^{\prime}, v^{\prime}$ and $u^{\prime} v^{\prime}$ and associated types of motion described above. Both Kim et al. (1971) and Corino and Brodkey (1969) agree that the bursting phenomenon is an important process for the Reynolds stress and thus the turbulent energy production. The largest contributions to

\begin{tabular}{|c|c|c|c|c|}
\hline quadrant & sign of $u^{\prime}$ & sign of $v^{\prime}$ & sign of $u^{\prime} v^{\prime}$ & type of motion \\
\hline 1 & + & + & + & Interaction(outward) \\
2 & - & + & - & Ejection \\
3 & - & - & + & Interaction(wall-ward) \\
4 & + & - & - & Sweep \\
\hline
\end{tabular}

Table 4.3: Classification of the fluid motion in $(u, v)$-plane.

the Reynolds stress are either from the ejection or the sweep events and this can be seen from the Figure 4.28 which shows the contribution to the Reynolds stress from each quadrant as a function of the non-dimensional wall-normal distance. The Reynolds shear stress are normalised by the local mean shear stress $\left\langle u^{\prime} v^{\prime}\right\rangle$. The present results are in good agreement with the simulation by Kim et al. (1987). At $y^{+} \approx 12$, usually associated with the maximum production of the turbulent kinetic energy, the contribution from the ejection motion equals that from the sweep motion and is about $60 \%$ of the $\left\langle u^{\prime} v^{\prime}\right\rangle$. The present data is slightly different from the experimental results obtained by Wallace et al. (1972) where they have the contribution from the ejection motion and sweep motion equals at $y^{+} \approx 15$ and is about $70 \%$ of the $\left\langle u^{\prime} v^{\prime}\right\rangle$. Departure from the balance point, $y^{+} \approx 12$, the sweep motion appears to be more dominant closer to the wall while the ejection motion more important away from the wall. As reported by Willmarth and $\mathrm{Lu}$ (1972), at $y^{+}=30$, the contribution from the ejection motion is $85 \%$ 


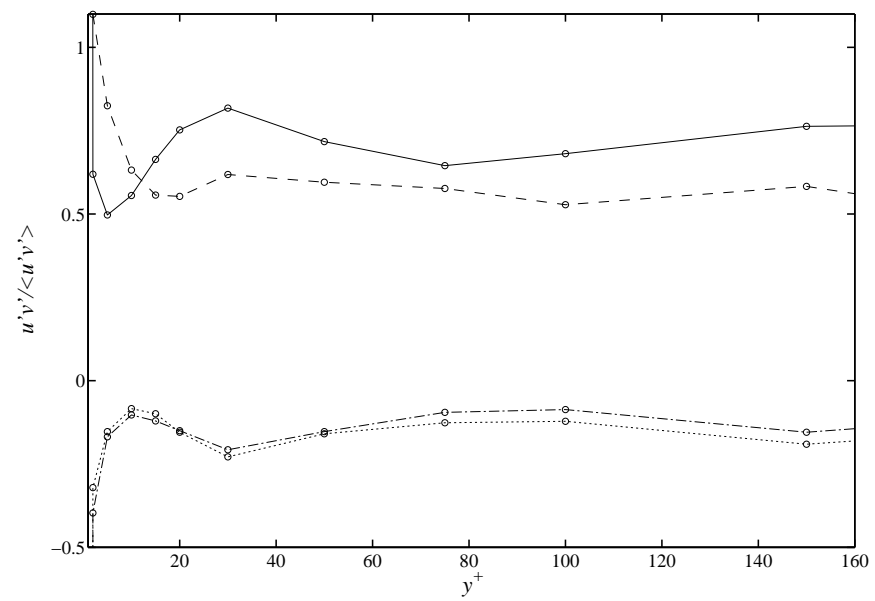

Figure 4.28: Fractional contribution to Reynolds shear stress $\left\langle u^{\prime} v^{\prime}\right\rangle$. — Ejection motion, - - - Sweep motion, -- - Outward motion, …… Wallward motion.

larger than the sweep motion. However, for the present case, it is only about $35 \%$ larger. The difference might lie in the high Reynolds number of the experiments by Willmarth and $\mathrm{Lu}$ (1972). The contributions from the other two types of motion, namely outward interaction and wall-ward interaction, are approximately equal through the entire boundary layer. At $y^{+} \approx 20$, the contributions from outward interaction and wall-ward interaction are equal.

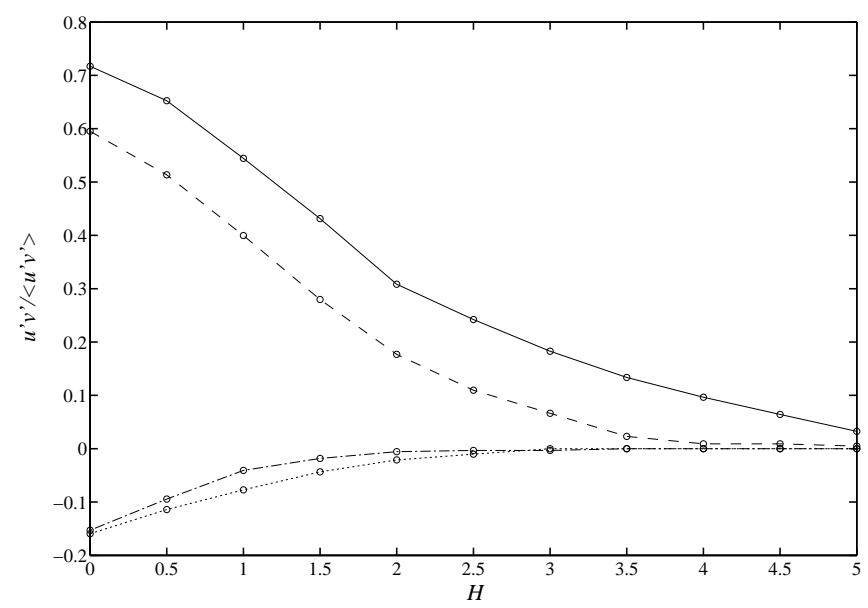

Figure 4.29: Fractional contribution to Reynolds shear stress $\left\langle u^{\prime} v^{\prime}\right\rangle$ as a function of threshold $H$. — Ejection motion, - - -Sweep motion, - - - Outward motion, ........ Wall-ward motion.

Fractional contributions from the four quadrants to the total Reynolds shear stress above a certain threshold value $H$ at $y^{+}=50$ is shown in Figure 
4.29. The purpose of using the threshold value is to single out the large contribution to $\left\langle u^{\prime} v^{\prime}\right\rangle$. The contributions that remain in the $(u, v)$ plane are the ones that occur outside a certain region bounded by four hyperbolae, $\frac{\left|u^{\prime} v^{\prime}\right|}{u_{r m s} v_{r m s}}<H$, in each quadrant.

\subsection{Scalar Transport Results}

Five different scalars (see Table 4.4) with either different boundary conditions or Prandtl numbers are simulated. Results are shown by comparing either different Prandtl numbers or different boundary conditions with the variation of the downstream positions. Note that all the scalars are transformed from $\theta$ to $1-\theta$ to simulate a cooling wall.

\begin{tabular}{|c|c|l|}
\hline scalar & boundary condition & \multicolumn{1}{|c|}{$\operatorname{Pr}$} \\
\hline$\theta_{1}$ & isothermal & 0.2 \\
$\theta_{2}$ & isothermal & 0.71 \\
$\theta_{3}$ & isoflux & 0.71 \\
$\theta_{4}$ & isothermal & 2.0 \\
$\theta_{5}$ & isoflux & 2.0 \\
\hline
\end{tabular}

Table 4.4: List of the different scalars with either different boundary conditions or Prandtl numbers.

\subsubsection{Reynolds' analogy}

For a fluid with $\operatorname{Pr}=1$ such that the kinematic viscosity for the momentum transfer equals the molecular diffusivity of the scalar transfer, the boundary layer governing equations (2.6) and (2.27) become identical for the mean velocity and the mean scalar. Thus the solutions to the boundary value problems for $\langle u\rangle$ and $\langle\theta\rangle$ must be equivalent which requires the wall-normal derivatives at the wall are the same after appropriate non-dimensionalisation

$$
\frac{\partial\langle u\rangle}{\partial y}=\frac{\partial\langle\theta\rangle}{\partial y} .
$$

Expressed in non-dimensional form, it is

$$
S t=\frac{c_{f}}{2},
$$

where $S t$ is the Stanton number, see definition in equation (4.27), and $c_{f}$ is the skin friction coefficient. According to Cebeci and Bradshaw (1984), although the analogy between the momentum transfer and scalar transfer is not exact, it is frequently close enough for a dimensionless quantity of the scalar field to be equated to the corresponding one in the flow field 
by multiplying with an "analogy factor". The most common factor is the Reynolds analogy factor defined by the ratio of the Stanton number to half of the skin friction coefficient. For the case of $\operatorname{Pr}=1$, the Reynolds' analogy factor $\frac{2 S t}{c_{f}}$ is unit.

\subsubsection{Mean scalar results}

The five mean scalar profiles normalised by the friction scalar $\theta_{\tau}$, which is defined by equation (2.58), are shown in Figure 4.30. As expected, these profiles look very similar to the streamwise velocity profile shown in Figure 4.2. The logarithmic region is not that clear for $\theta_{1}$ as shown in Figure 4.30 (a). If the $\operatorname{Pr}$ number is even lower, the logarithmic region will not exist due to the thickening of the conductive sub-layer (Kasagi and Ohtsubo, 1993). An increase of the mean scalar value with the increasing Prandtl number is clearly observed which indicates that the scalar boundary layer thickens with the decreasing Prandtl number.

The mean profile of $\theta_{2}$, i.e. isothermal boundary condition and $\operatorname{Pr}=$ 0.71, together with the empirical formula suggested by Kader (1981) and DNS results by Kawamura et al. (1998) is plotted in Figure 4.31. Inside the conductive sub-layer, the calculated mean scalar profile follows the relation of

$$
\theta^{+}=\operatorname{Pry}^{+}
$$

fairly well. In the log region, all the profiles collapse with the log-law

$$
\theta^{+}=2.195 \ln y^{+}+13.2 \operatorname{Pr}-5.66
$$

suggested by Bejan (1995). The formula suggested by Kader (1981) reads

$\theta^{+}=\operatorname{Pry}^{+} \exp (-\Gamma)+\left\{2.12 \ln \left[\left(1+y^{+}\right) \frac{2.5\left(2-\frac{y}{\delta}\right)}{1+4\left(1-\frac{y}{\delta}\right)^{2}}\right]+\beta(\operatorname{Pr})\right\} \exp \left(-\frac{1}{\Gamma}\right)$

where

$$
\Gamma=\frac{10^{-2}\left(\operatorname{Pry}^{+}\right)^{4}}{1+5 \operatorname{Pr}^{3} y^{+}}
$$

and

$$
\beta(\operatorname{Pr})=\left(3.85 \operatorname{Pr}^{\frac{1}{3}}-1.3\right)^{2}+2.12 \ln \operatorname{Pr},
$$

where $\delta$ is the $99 \%$ boundary layer thickness. Kader (1981) proposed this formula after investigating several experimental data. This formula is shown to be a function of the molecular Prandtl number $P r$, Reynolds number $R e$ as well as the boundary layer thickness $\delta$. As shown in the figure, the agreement between the computed results and Kader's formula is very good. The mean scalar profile also agrees with the DNS results from Kawamura et al. (1998) very well in spite of the different boundary conditions. This 


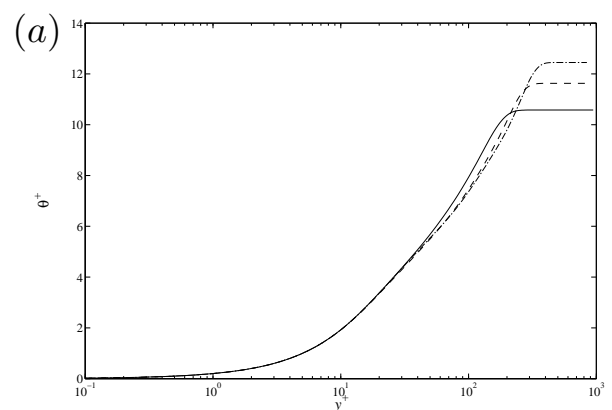

(b)

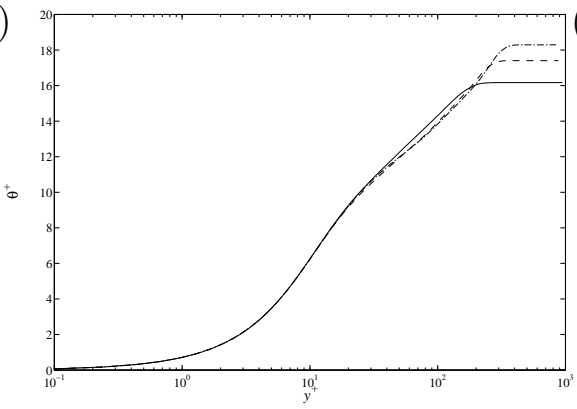

$(d)$

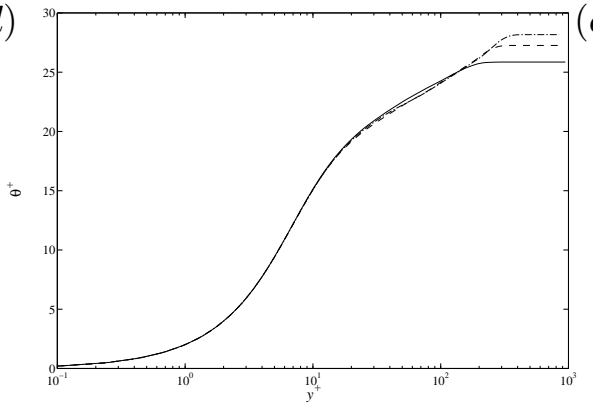

(c)

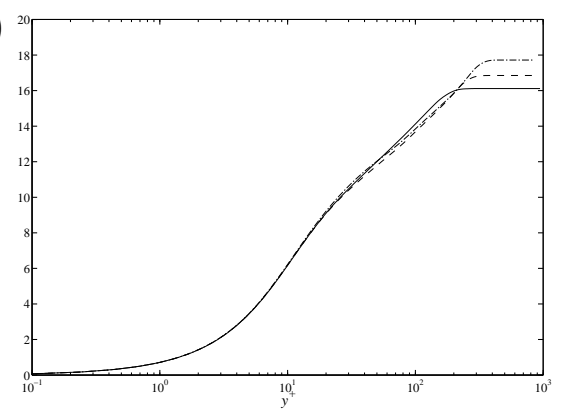

e)

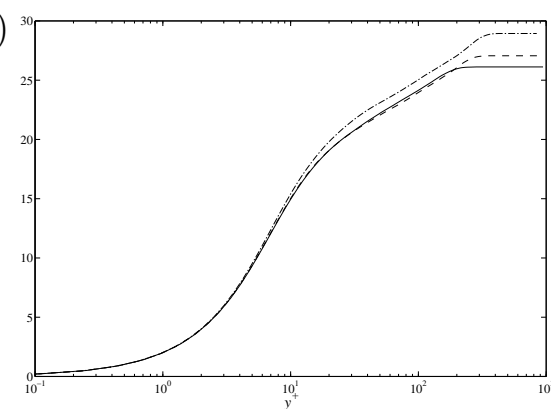

Figure 4.30: Mean scalar profiles at different downstream positions. — at $R e_{\theta}=396,---$ at $R e_{\theta}=628,-$ - at $R e_{\theta}=830$. (a) $\theta_{1}$, (b) $\theta_{2},(c) \theta_{3},(d)$ $\theta_{4},(e) \theta_{5}$.

verifies the previous findings by Tiselj et al. (2001b) that the boundary condition for scalar does not affect the mean scalar profile.

The Stanton number $S t$ for a scalar boundary layer is analogous to the skin friction coefficient for a momentum boundary layer. This nondimensional scalar transfer coefficient is defined by

$$
S t=\frac{q_{w}}{\rho U_{\infty} C_{p}\left(\theta_{w}-\theta_{\infty}\right)},
$$

where $q_{w}$ is the rate of the scalar transfer from the wall to the flow, $\rho$ is the density of the fluid, $U_{\infty}$ is the free-stream velocity, $C_{p}$ is the specific scalar, $\theta_{w}$ and $\theta_{\infty}$ are the scalar concentrations at the wall and in the freestream, respectively. The variation of the Stanton number with different downstream positions is shown in Figure 4.32. The lower and upper dashed 


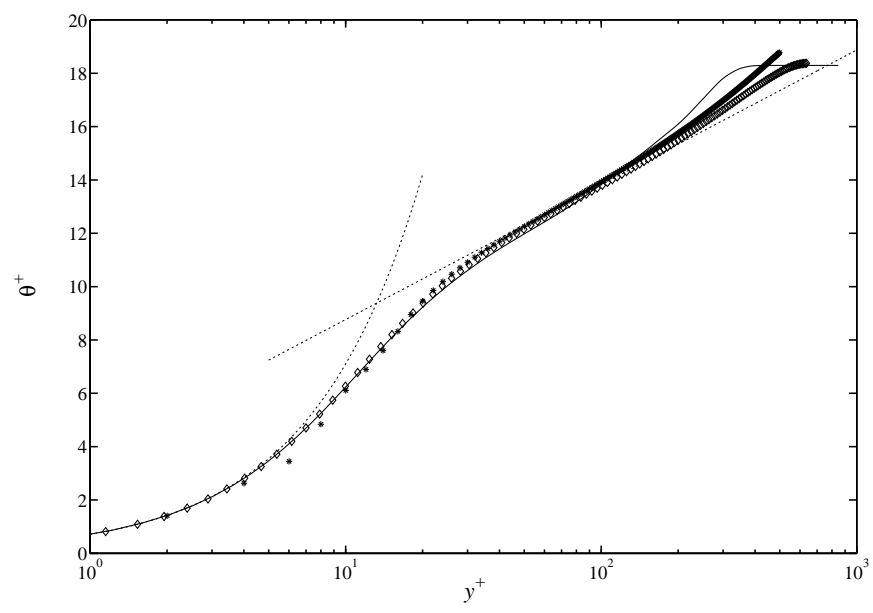

Figure 4.31: Mean scalar profile compared with other numerical simulation and empirical data. $-\theta_{2}$ at $R e_{\theta}=830, \cdots \cdots$ Log-law, $*$ Kader (1981), $\diamond$ Kawamura et al. (1998) at $R e_{\tau}=395$.

lines are the laminar and modified form of the turbulent solutions suggested by Kays and Crawford (1993).

It is not surprising that the Stanton number profiles look very similar to the one of the skin friction coefficient, see Figure 4.6, for the isothermal case. An overshoot is also observed in each plot. However, for the isoflux boundary condition, one interesting thing is found that the overshoot vanishes.

Another important parameter in scalar transfer is the turbulent Prandtl number $P r_{t}$. The turbulent Prandtl number plays an important role in the prediction of the near wall scalar transfer using turbulence models and is defined as the ratio of the turbulent eddy viscosity $\nu_{t}$ to the eddy diffusivity $\alpha_{t}$,

$$
\operatorname{Pr}_{t}=\frac{\nu_{t}}{\alpha_{t}}
$$

where $\nu_{t}$ and $\alpha_{t}$ are defined by (strictly only for parallel flows)

$$
\nu_{t}=-\frac{\left\langle u^{\prime} v^{\prime}\right\rangle}{\frac{\partial\langle u\rangle}{\partial y}}
$$

and

$$
\alpha_{t}=-\frac{\left\langle v^{\prime} \theta^{\prime}\right\rangle}{\frac{\partial\langle\theta\rangle}{\partial y}} .
$$

The Reynolds' analogy is the simplest model for the turbulent Prandtl number since it leads to the equivalence of the eddy viscosity for the momentum transfer and the eddy diffusivity of the scalar transfer such that the $P r_{t}=1$ (Kays and Crawford, 1993).

To analyse the near wall asymptotic behaviour of the turbulent scalar statistics, we expand the velocity and scalar distributions in Taylor series. 

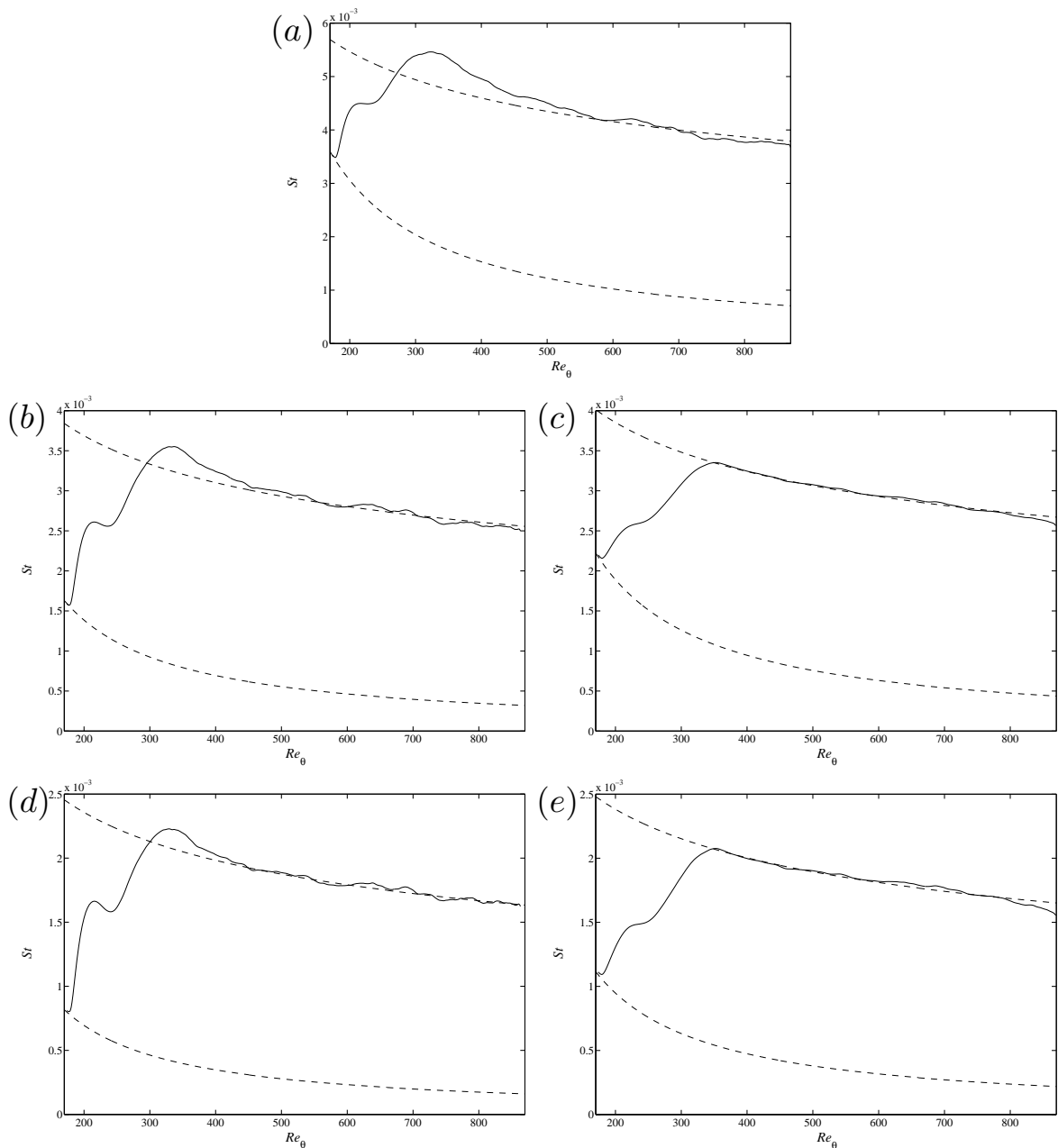

Figure 4.32: Stanton number $S t$ versus the Reynolds number. - Present DNS, - - Kays and Crawford (1993). (a) $\theta_{1},(b) \theta_{2},(c) \theta_{3},(d) \theta_{4},(e) \theta_{5}$.

Considering the continuity and dynamic equations under the no-slip and isothermal and isoflux boundary conditions, $\langle u\rangle, u^{\prime}, v^{\prime},\langle\theta\rangle, \theta^{\prime}$ are expressed in the following form (Monin and Yaglom, 1971)

$$
\begin{aligned}
\langle u\rangle & =a_{1} y+a_{4} y^{4}+\cdots, \\
u^{\prime} & =b_{1} y+b_{2} y^{2}+\cdots, \\
v^{\prime} & =c_{2} y^{2}+c_{3} y^{3}+\cdots .
\end{aligned}
$$

For an isothermal boundary condition, $\langle\theta\rangle, \theta^{\prime}$ are expanded as

$$
\begin{aligned}
\langle\theta\rangle & =d_{0}+d_{1} y+d_{4} y^{4}+\cdots, \\
\theta^{\prime} & =e_{1} y+e_{2} y^{2}+\cdots
\end{aligned}
$$

while in the case of an isoflux boundary condition, the scalar and its fluctu- 
ation are

$$
\begin{aligned}
\langle\theta\rangle & =f_{0}+f_{1} y+f_{3} y^{3}+\cdots, \\
\theta^{\prime} & =g_{0}+g_{1} y+g_{3} y^{3}+\cdots,
\end{aligned}
$$

where the coefficients $a_{i}, b_{i}, c_{i}, d_{i}, e_{i}, f_{i}$ and $g_{i}(i=0,1,2, \ldots)$ are functions of $x, z$ and $t$. Note that the second and third order terms in the mean velocity vanish together with the first order term in the wall-normal velocity fluctuation. The second and third order terms in the mean scalar and the second order term in the scalar fluctuation vanish for an isothermal boundary condition. On the other hand, only the second order term in the mean scalar and the scalar fluctuation vanish for an isoflux boundary condition.

From the equations (4.31) and (4.32) the streamwise and wall-normal scalar fluxes for the isothermal boundary condition are given by

$$
\begin{aligned}
& \left\langle u^{\prime} \theta^{\prime}\right\rangle=b_{1} e_{1} y^{2}+\cdots, \\
& \left\langle v^{\prime} \theta^{\prime}\right\rangle=c_{2} e_{1} y^{3}+\cdots .
\end{aligned}
$$

On the other hand, for the isoflux boundary condition, the streamwise and wall-normal scalar fluxes derived using equation (4.31) and (4.33) are

$$
\begin{aligned}
\left\langle u^{\prime} \theta^{\prime}\right\rangle & =b_{1} g_{0} y+\cdots, \\
\left\langle v^{\prime} \theta^{\prime}\right\rangle & =c_{2} g_{0} y^{2}+\cdots .
\end{aligned}
$$

Substitution of equation (4.34) and (4.35) into equation (4.29) and (4.30) yields the relations of $\mathrm{Pr}_{t}$ for isothermal and isoflux boundary conditions

$$
\operatorname{Pr}_{t}=\frac{b_{1} c_{2} d_{1}}{a_{1} c_{2} e_{1}}+\cdots
$$

and

$$
P r_{t}=\frac{b_{1} c_{2} f_{1}}{a_{1} c_{2} g_{0}} y+\cdots
$$

From the Figure 4.33, it is clear that the limiting behaviours of $P r_{t}$ for both the isothermal and isoflux boundary conditions are well predicted in the present DNS. The variation of $P r_{t}$ at $R e_{\theta}=830$ with the wall-normal distances is plotted in Figure 4.34. The turbulent Prandtl number is often assumed to be a constant value which is independent of the wall-normal distance and the molecular Prandtl number. However, the dependence on the wall-normal distance and $\operatorname{Pr}$ has long been a subject of many investigations (Kestin and Richardson, 1963; Kader and Yaglom, 1972). Kays (1994) proposed a correlation for $\mathrm{Pr}_{t}$ based on several independent experiments. It showed a rather steep increase as the wall was approached. For the present DNS, the $\operatorname{Pr}_{t}$ for the scalars $\theta_{2}$ and $\theta_{4}$ with the isothermal boundary condition approach approximately a constant value of about 1.1 at the wall 


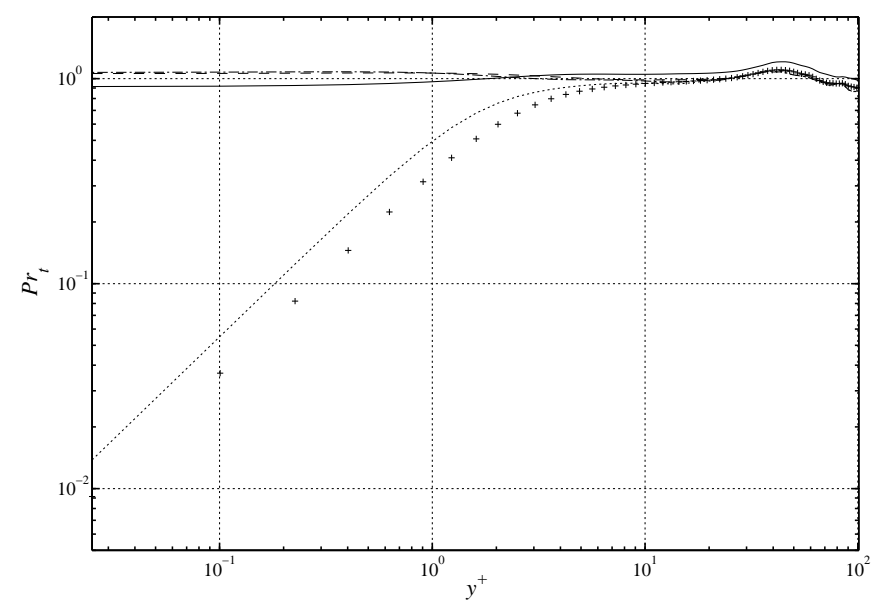

Figure 4.33: Near wall behaviour of the turbulent Prandtl number $P r_{t}$ at $\operatorname{Re}_{\theta}=830 .-\theta_{1},---\theta_{2},+\theta_{3},-\cdot-\theta_{4}, \cdots \cdots \cdot \theta_{5}$.

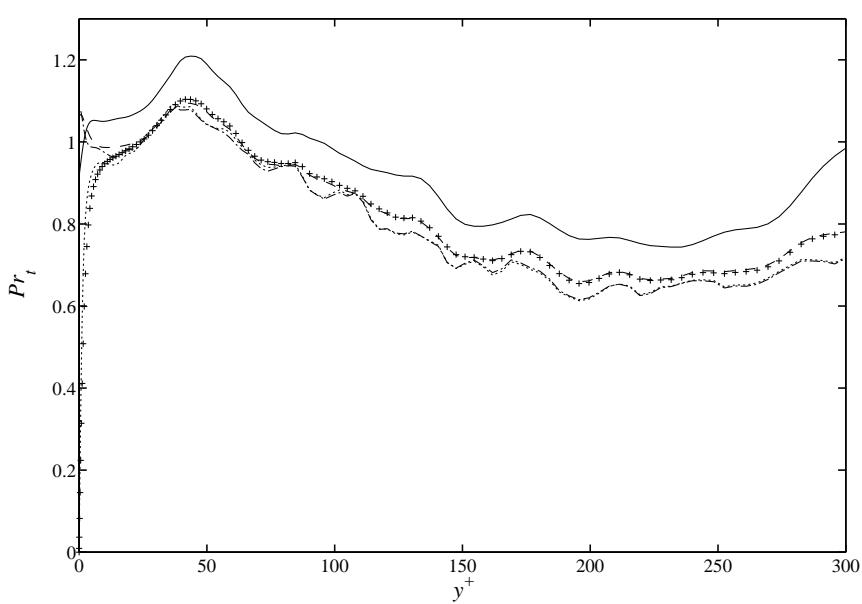

Figure 4.34: Turbulent Prandtl number $\operatorname{Pr}_{t}$ versus the wall-normal distance at $\operatorname{Re}_{\theta}=830 .-\theta_{1},---\theta_{2},+\theta_{3},-\cdot-\theta_{4}, \cdots \cdots \theta_{5}$.

which is independent on the molecular Prandtl number. This behaviour is also reported by many previous studies, e.g. Kong et al. (2000) and Jacobs and Durbin (2000) for turbulent boundary layer flow and Kim and Moin (1989) and Kasagi et al. (1992) for fully developed turbulent channel flow. However, the profile of the scalar $\theta_{1}$ with $\operatorname{Pr}=0.2$ is different from the others. The value of the $\operatorname{Pr}_{t}$ at the wall for $\theta_{1}$ is about 0.95 and increases from the wall to the peak value of 1.2 at $y^{+} \approx 45$. And throughout the boundary layer, clear difference with the other profiles can be seen. This might be a low $\operatorname{Pr}$ effect. A similar behaviour was observed by Kasagi and Ohtsubo (1993) for a channel flow of a much lower $\operatorname{Pr}$ of 0.025 .

For all the three cases of the isothermal boundary condition considered 
here, the values of the turbulent Prandtl number remain above 1 until $y^{+} \approx$ $90-100$, which is larger than the value of $y^{+} \approx 60-70$ reported by Kong et al. (2000), and less than 1 as $y^{+}>100$. On the contrary, the profiles of the turbulent Prandtl number for the isoflux wall are different from those of the isothermal wall. In this case, the turbulent Prandtl numbers are zero at the wall which can be seen from equation (4.37). Far away from the wall, Figure 4.34 suggests that the distributions of $\operatorname{Pr}_{t}$ for $\operatorname{Pr}=0.71$ and 2 with different boundary conditions follow each other closely. This feature is in accordance with the previous studies by Jischa and Rieke (1979) and Antonia and Kim (1991).

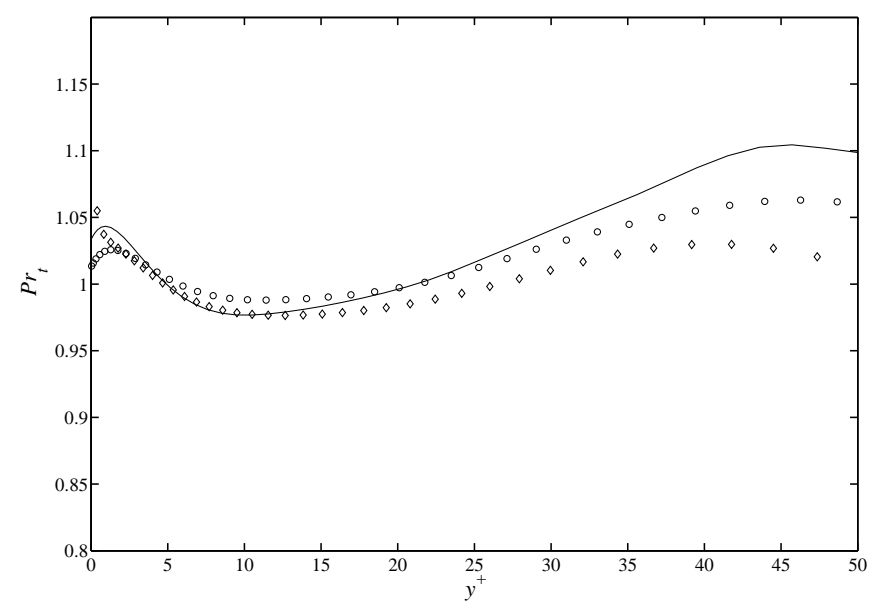

Figure 4.35: Comparison of $P r_{t}$ with other DNS data. $-\theta_{2}$ at $R e_{\theta}=628$, $\circ$ Kasagi et al. (1992) at $R e_{\tau}=150, \diamond$ Kawamura et al. (1998) at $R e_{\tau}=180$.

A comparison of the $P r_{t}$ of $\theta_{2}$ with the DNS data from Kasagi et al. (1992) and Kawamura et al. (1998) at the same Prandtl number, i.e. $\operatorname{Pr}=$ 0.71, is plotted in Figure 4.35. Both these two simulations are channel flow with the Reynolds numbers being $R e_{\tau}=150$ and $R e_{\tau}=180$, respectively. As seen from the plot, in the near wall region, the agreement with both the simulations is good.

Similarly as for the flow field, we can also define the corresponding boundary layer thickness measurements for the scalar field, i.e. the $99 \%$ scalar boundary layer thickness $\delta_{99}^{\theta}$, the scalar displacement thickness $\delta_{1}^{\theta}$ and the scalar momentum thickness $\delta_{2}^{\theta}$. The $\delta_{99}^{\theta}$ is defined as

$$
\langle\theta\rangle\left(y=\delta_{99}^{\theta}\right)=0.99 \theta_{\infty},
$$

while the scalar displacement thickness $\delta_{1}^{\theta}$ and the scalar momentum thickness $\delta_{2}^{\theta}$ are defined by

$$
\delta_{\theta}^{*} \equiv \delta_{1}^{\theta}=\int_{0}^{\infty}\left(1-\frac{\langle\theta\rangle-\theta_{w}}{\theta_{\infty}-\theta_{w}}\right) \mathrm{d} y
$$


and

$$
\theta_{\theta} \equiv \delta_{2}^{\theta}=\int_{0}^{\infty} \frac{\langle\theta\rangle-\theta_{w}}{\theta_{\infty}-\theta_{w}}\left(1-\frac{\langle\theta\rangle-\theta_{w}}{\theta_{\infty}-\theta_{w}}\right) \mathrm{d} y,
$$

where $\theta_{w}$ and $\theta_{\infty}$ are the scalar concentrations at the wall and in the freestream, respectively. The profiles of $99 \%$ scalar boundary layer thickness as well as the scalar displacement thickness $\delta_{1}^{\theta}$ and the scalar momentum thickness $\delta_{2}^{\theta}$ are shown in Figures 4.36 and 4.37 .
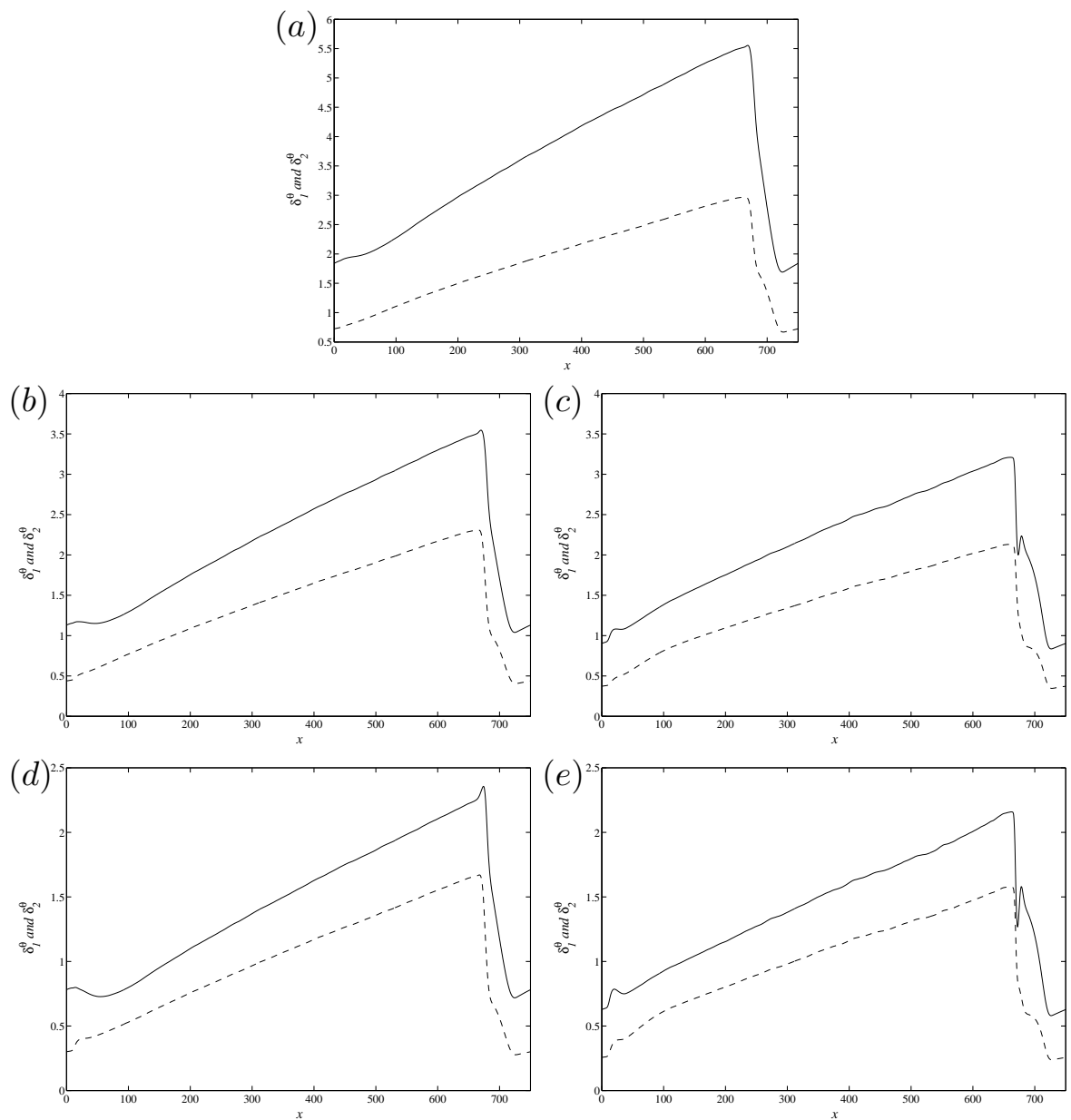

Figure 4.36: Different measures of the scalar boundary layer thickness versus the downstream positions. - - Scalar momentum thickness $\delta_{2}^{\theta}$. (a) $\theta_{1},(b) \theta_{2},(c) \theta_{3},(d) \theta_{4},(e) \theta_{5}$.

\subsubsection{Turbulent statistics}

The root-mean-square (RMS) values of the scalar fluctuations normalised by the friction scalar $\theta_{\tau}$ are shown in Figure 4.38. The wall-normal distance is normalised by $\frac{\nu}{u_{\tau}}$. Note that the RMS values at the walls in Figure 4.38 

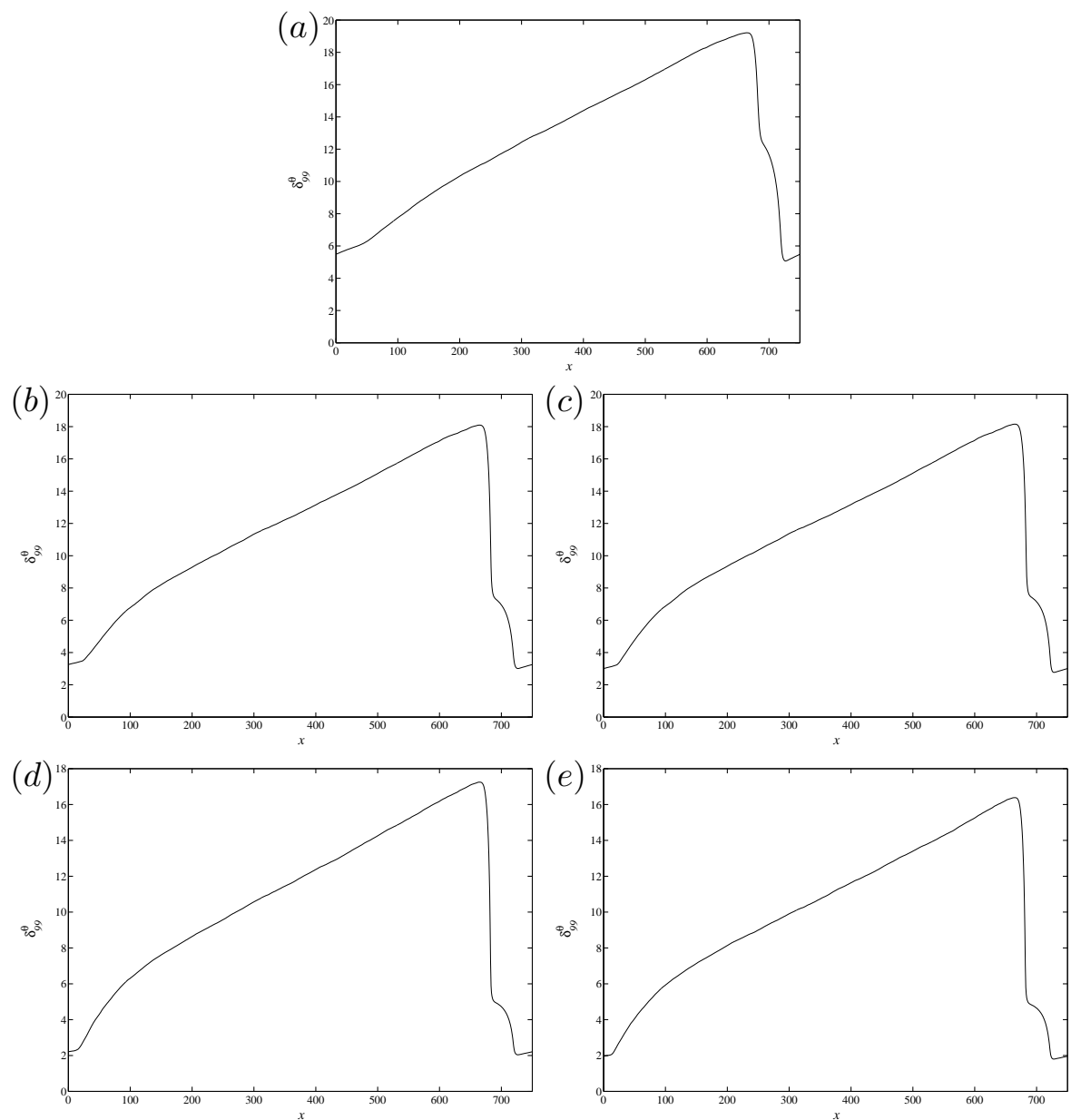

Figure 4.37: $99 \%$ scalar boundary layer thickness versus the downstream positions. (a) $\theta_{1},(b) \theta_{2},(c) \theta_{3},(d) \theta_{4},(e) \theta_{5}$.

$(c)$ and $(e)$ are non-zero due to the isoflux boundary condition. A plot of the wall values of the RMS of the scalar fluctuations for the isoflux boundary condition is shown in Figure 4.39.

As can be seen from the plot, the wall values of the RMS of the scalar fluctuations which employed the isoflux boundary conditions are increasing as the Reynolds number is increased. However this is different with a previous study by Kong et al. (2000) who reported that the wall values of the RMS of the scalar fluctuations are constant and independent on the Reynolds number range in their study.

For each scalar of the isothermal boundary condition, the RMS values of the scalar fluctuations with different Reynolds numbers collapse with each other for $y^{+}<10$. The peak values increase with an increase of the Reynolds number. In the outer region, the profiles of the RMS scalar fluctuations are different from each other owing to the Reynolds number effect. This 

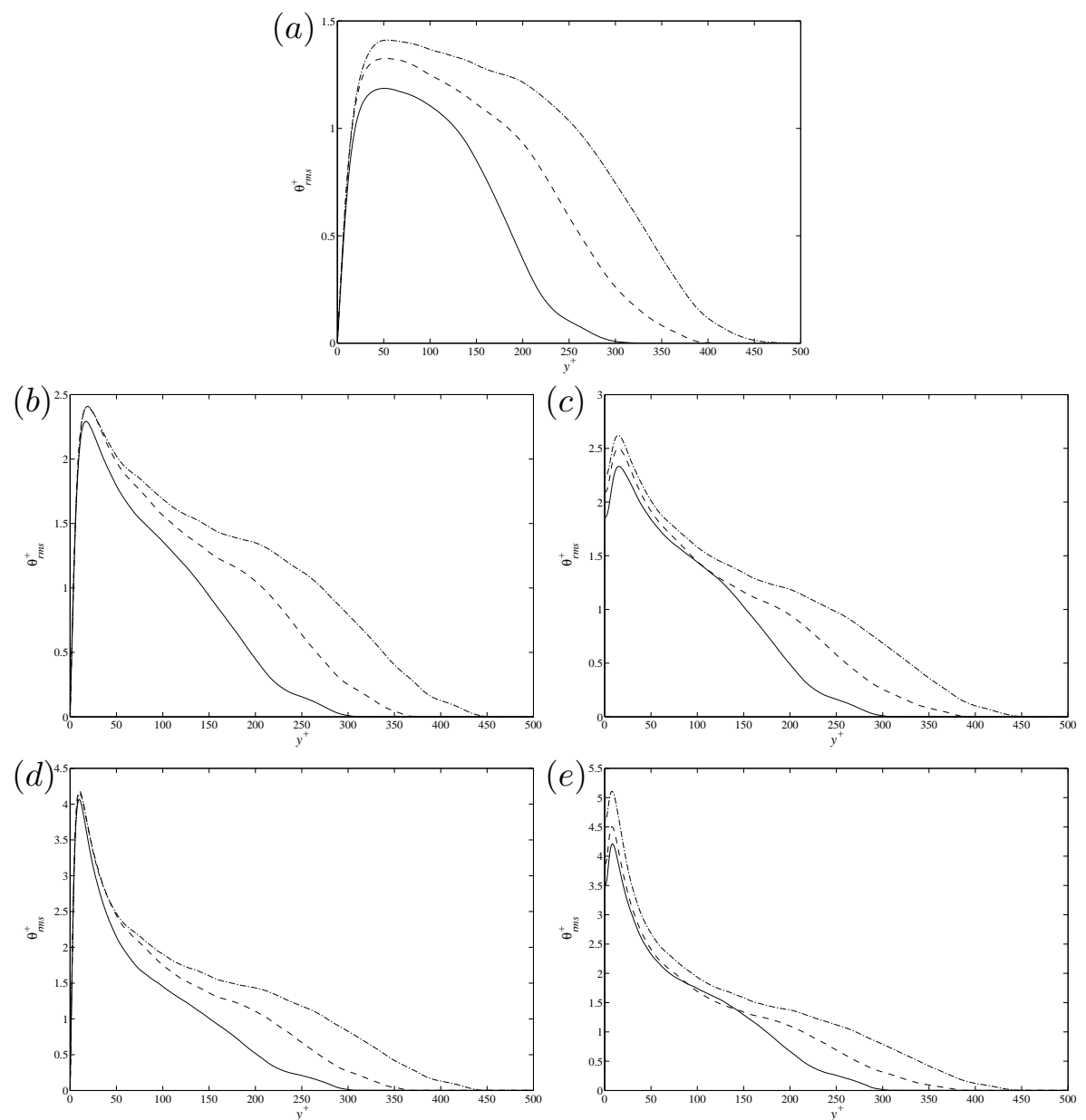

Figure 4.38: RMS values of the scalar fluctuation at different downstream positions. - at $R e_{\theta}=396,---$ at $R e_{\theta}=628,-\cdot-$ at $R e_{\theta}=830$. (a) $\theta_{1}$, (b) $\theta_{2},(c) \theta_{3},(d) \theta_{4},(e) \theta_{5}$.

behaviour is exactly the same as the RMS value of the streamwise velocity. The maximum RMS values of the scalar fluctuation increase as the $\operatorname{Pr}$ is increasing, the peak value of the RMS values of the scalar fluctuation at $\operatorname{Pr}=0.2$ is about 3 times smaller than that at $\operatorname{Pr}=2$. It is also observed that the peak position moves away from the wall as the Prandtl number is decreased.

A comparison of the RMS values of the scalar fluctuation with different boundary conditions at $R e_{\theta}=628$ is plotted in Figure 4.40. Like the profiles of the turbulent Prandtl number, differences can only be seen in the near wall region. Far away from the wall the influence of the scalar boundary conditions is negligible. According to Tiselj et al. (2001a), the influence ranges of the scalar boundary conditions are at $y^{+} \approx 15$ for $\operatorname{Pr}=0.71$ and $y^{+} \approx 6-8$ for $\operatorname{Pr}=5-7$. However, the present DNS predict a little bit 


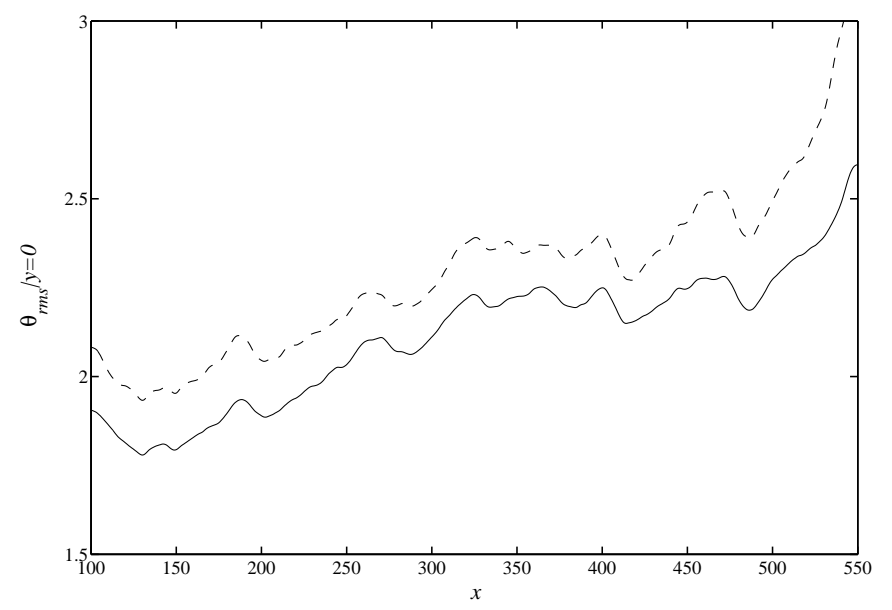

Figure 4.39: RMS values at the wall of the scalar fluctuations at different downstream positions with $R e_{\theta}=830 .-\theta_{3},---\theta_{5}$.

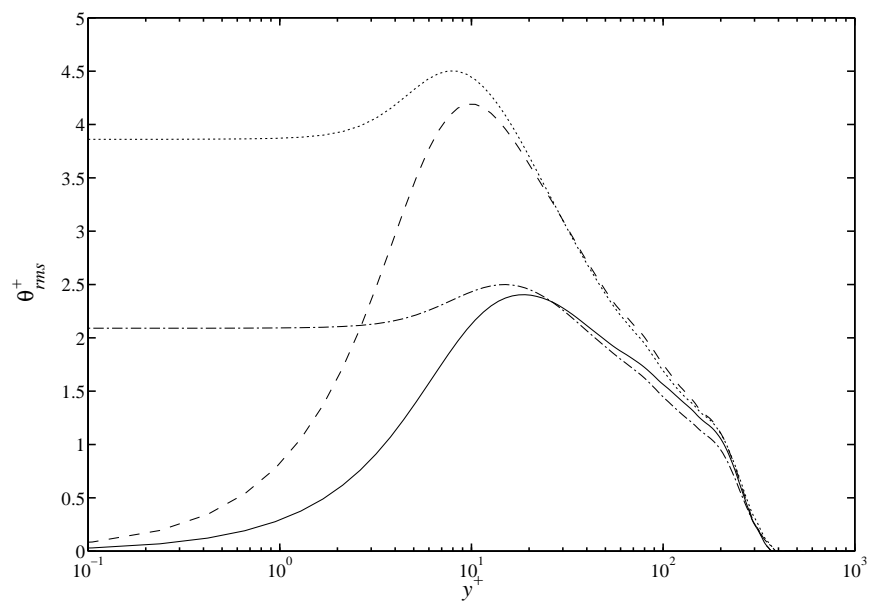

Figure 4.40: Comparison of the RMS values of the scalar fluctuation with different boundary conditions. $-\theta_{2},-\cdot-\theta_{3},---\theta_{4}, \cdots \cdots \theta_{5}$.

larger values than those reported by Tiselj et al. (2001a).

The RMS value of $\theta_{2}$ compared with other DNS data from Kasagi et al. (1992) and Kawamura et al. (1998) is shown in Figure 4.41. The agreement with the DNS data from Kasagi et al. (1992) is only good very close to the wall. Very good agreement can be observed with the data from Kawamura et al. (1998). A higher peak value is obtained by Kawamura et al. (1998) which is due to the higher Reynolds number. The maximum scalar fluctuation of $\theta_{2}$ occurs at $y^{+} \approx 18$, which is the same as the other two simulations and also as the same as the one reported by Lu and Hetsroni (1995). This position is slightly higher than the location of maximum streamwise velocity fluctuations which is $y^{+} \approx 14$ and consistent with the previous studies by 


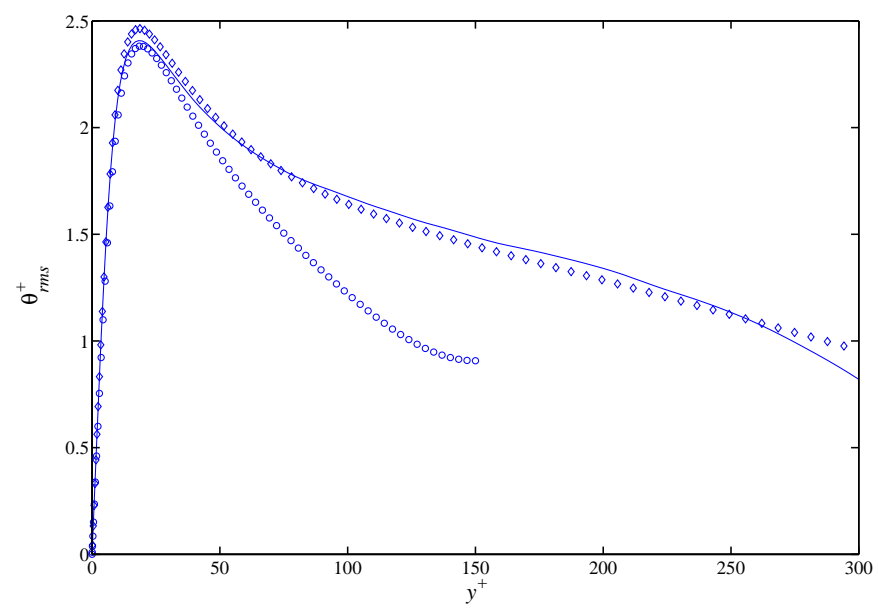

Figure 4.41: Comparison of the RMS values of the scalar fluctuation with other DNS data. $-\theta_{2}$ at $R e_{\theta}=830$, ○ Kasagi et al. (1992) at $R e_{\tau}=150$, $\diamond$ Kawamura et al. (1998) at $R e_{\tau}=395$.

Kong et al. (2000) and Kim and Moin (1989).

The wall-normal distribution of the streamwise and wall-normal scalar fluxes are shown in Figures 4.42 and 4.43. The scalar fluxes are nondimensionlised by $u_{\tau} \theta_{\tau}$ and the wall-normal distance by $\frac{u_{\tau}}{\nu}$. From the plots, we see that as the Prandtl number is decreased, the turbulent scalar flux also becomes smaller due to the thickening of the conductive sub-layer. The wall-normal component is smaller than the streamwise scalar flux by an order of magnitude at $\operatorname{Pr}=2$. However, as $\operatorname{Pr}$ is decreased, the difference between the streamwise and wall-normal components become smaller. This is due to the fact that turbulent scalar fluxes are more isotropic when the $\mathrm{Pr}$ is small. This is consistent with the previous study by Kasagi and Ohtsubo (1993). We also found that the peak position of the scalar flux moves further away from the wall when the Prandtl number is smaller and the peak position value of the streamwise scalar flux is closer to the wall compared to that of the wall-normal scalar flux. This may be due to the fact that the wall-normal scalar flux is heavily damped in the near wall region. Again, the difference due to the different boundary conditions is only significant in the near wall region.

In addition, by comparing the wall-normal scalar flux of $\theta_{2}$ and the Reynolds shear stress $\left\langle u^{\prime} v^{\prime}\right\rangle$ one can find that the profiles of these two quantities are almost identical. The similar distributions reveal that they are likely to be generated by similar mechanisms. 

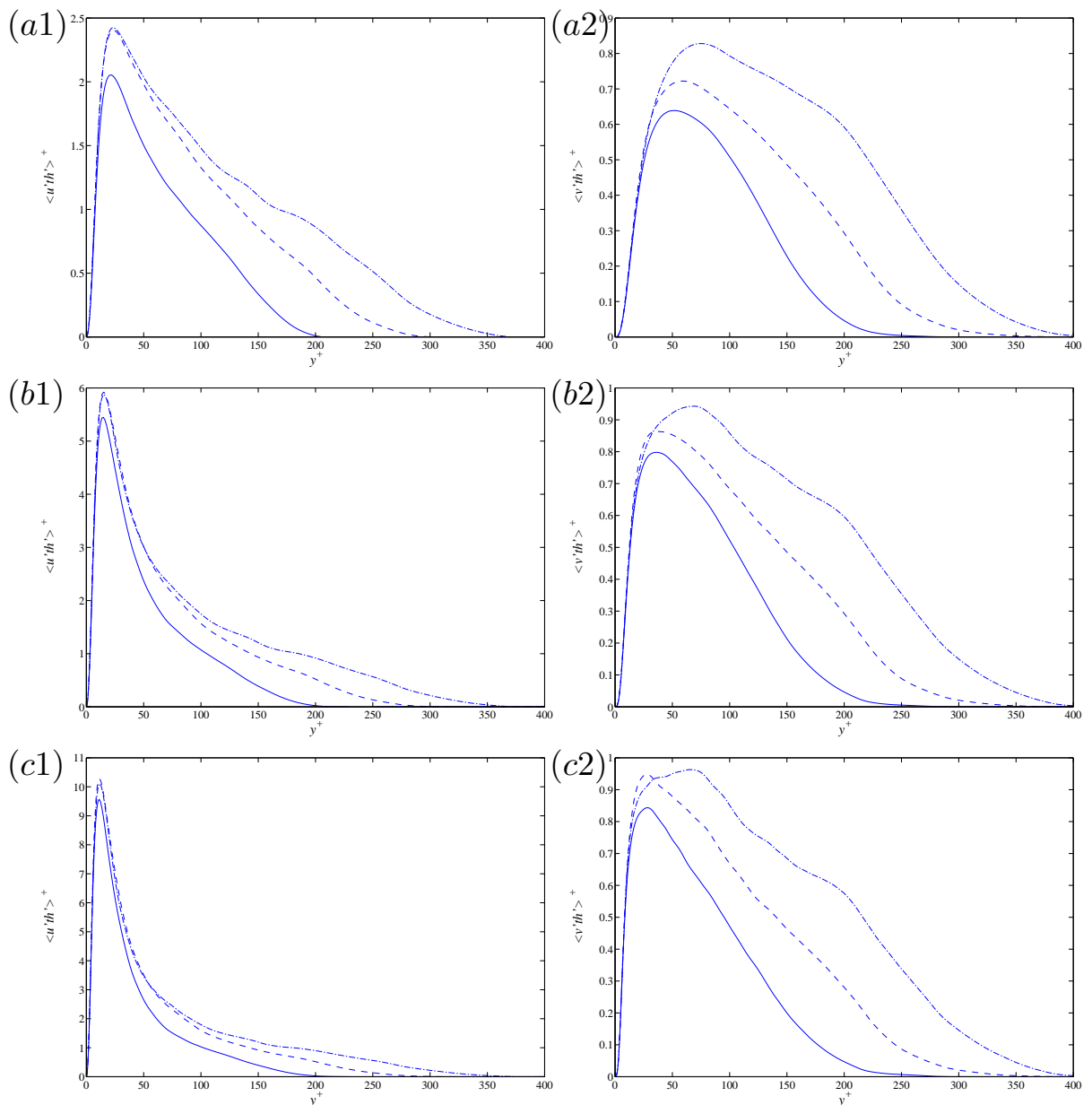

Figure 4.42: Streamwise and wall-normal scalar flux fluctuations for isothermal boundary condition at different downstream positions. —at $R e_{\theta}=$ 396, - - - at $R e_{\theta}=628,-$ - at $\operatorname{Re}_{\theta}=830$. (a) $\theta_{1}$, (b) $\theta_{2}$, (c) $\theta_{4}$. (1) Streamwise scalar flux, (2) Wall-normal scalar flux.

\subsubsection{Scalar flux budget}

Figure 4.44 shows the budget for the fluctuating scalar variance $k_{\theta}$, which is defined by

$$
k_{\theta}=\frac{1}{2}\left\langle\theta^{\prime} \theta^{\prime}\right\rangle
$$

Each term in the budget equation is normalised by $\frac{u_{\tau}^{2} \theta_{\tau}^{2}}{\nu}$. From the plots we have seen that the convection term is negligible throughout the boundary layer. The contribution from the molecular diffusion to the budget is confined near the wall. Although the production and dissipation are two major terms for all the three Prandtl numbers calculated here, the turbulent and molecular diffusion terms play a more important role throughout a signifi- 

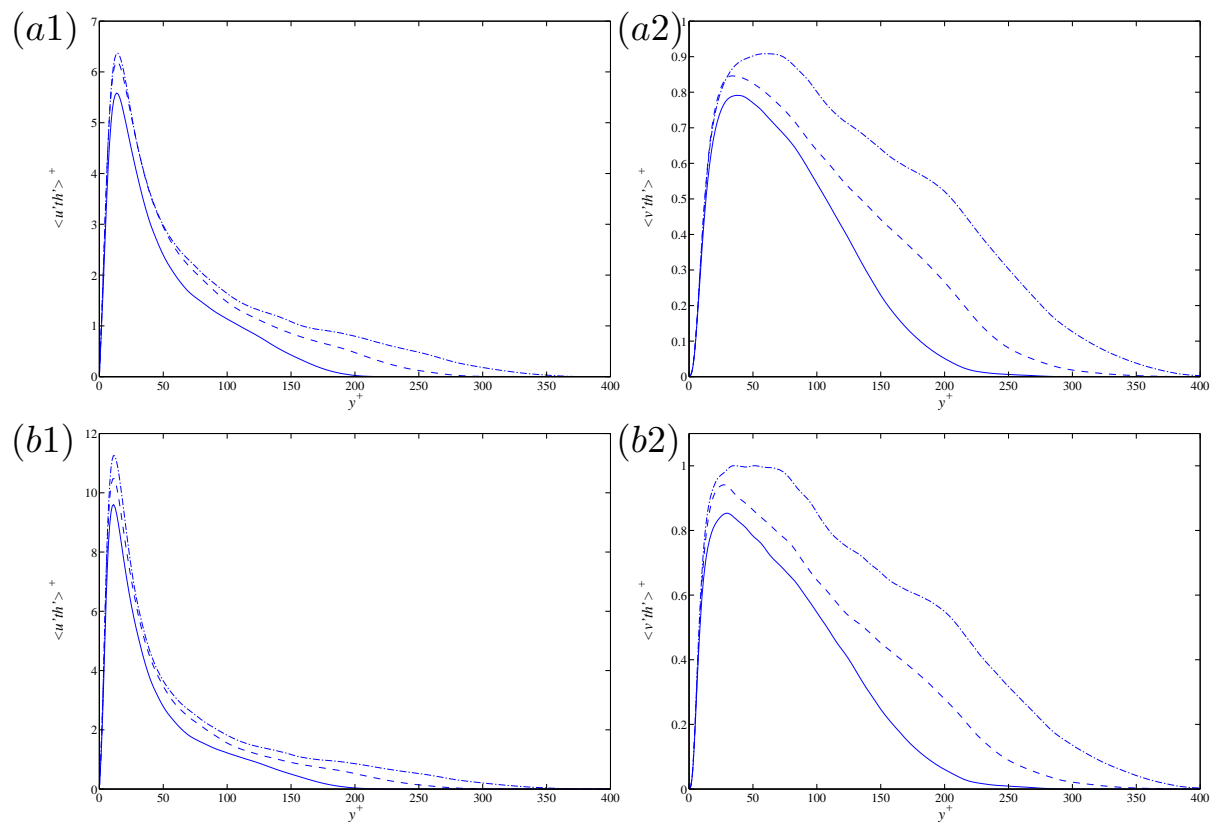

Figure 4.43: Streamwise and wall-normal scalar flux fluctuations for isoflux boundary condition at different downstream positions. - at $R e_{\theta}=396$, - - - at $R e_{\theta}=628,-$ - at $R e_{\theta}=830$. (a) $\theta_{3}$, (b) $\theta_{5}$. (1) Streamwise scalar flux, (2) Wall-normal scalar flux.

cant portion of the boundary layer with increasing Prandtl number. This is also reported previously by Jacobs and Durbin (2000). For the dominant terms, the peak values increase and the peak positions move towards the wall as $\operatorname{Pr}$ is increased. As can clearly be seen from the plots, the molecular diffusion and dissipation terms are strongly influenced by the boundary conditions near the wall which implies that the influence of the scalar boundary conduction in the near wall region can not be neglected.

A comparison of the fluctuating scalar variance for $\theta_{2}$ is displayed in Figure 4.45 with the channel DNS data of Kawamura et al. (1998) which is obtained under the same boundary condition and $\mathrm{Pr}$ as the present DNS. Very good agreement with the DNS data of Kawamura et al. (1998) can be seen in the near wall region.

The budgets for the streamwise and wall-normal scalar fluxes are shown in Figures 4.46 and 4.47. For the streamwise scalar flux $\left\langle u^{\prime} \theta^{\prime}\right\rangle$ budget, the production and dissipation terms are dominant in the whole region and the molecular diffusion term is only noticeable in the near wall region. The scalar pressure gradient correlation term is small and always lies on the loss side while the turbulent diffusion term behaves the same as in the budget for the fluctuating scalar variance.

For the wall-normal scalar flux $\left\langle v^{\prime} \theta^{\prime}\right\rangle$ budget, the production term is negative in this case. It is well known that, in case of the fluid with $\operatorname{Pr} \geq 0.7$, 

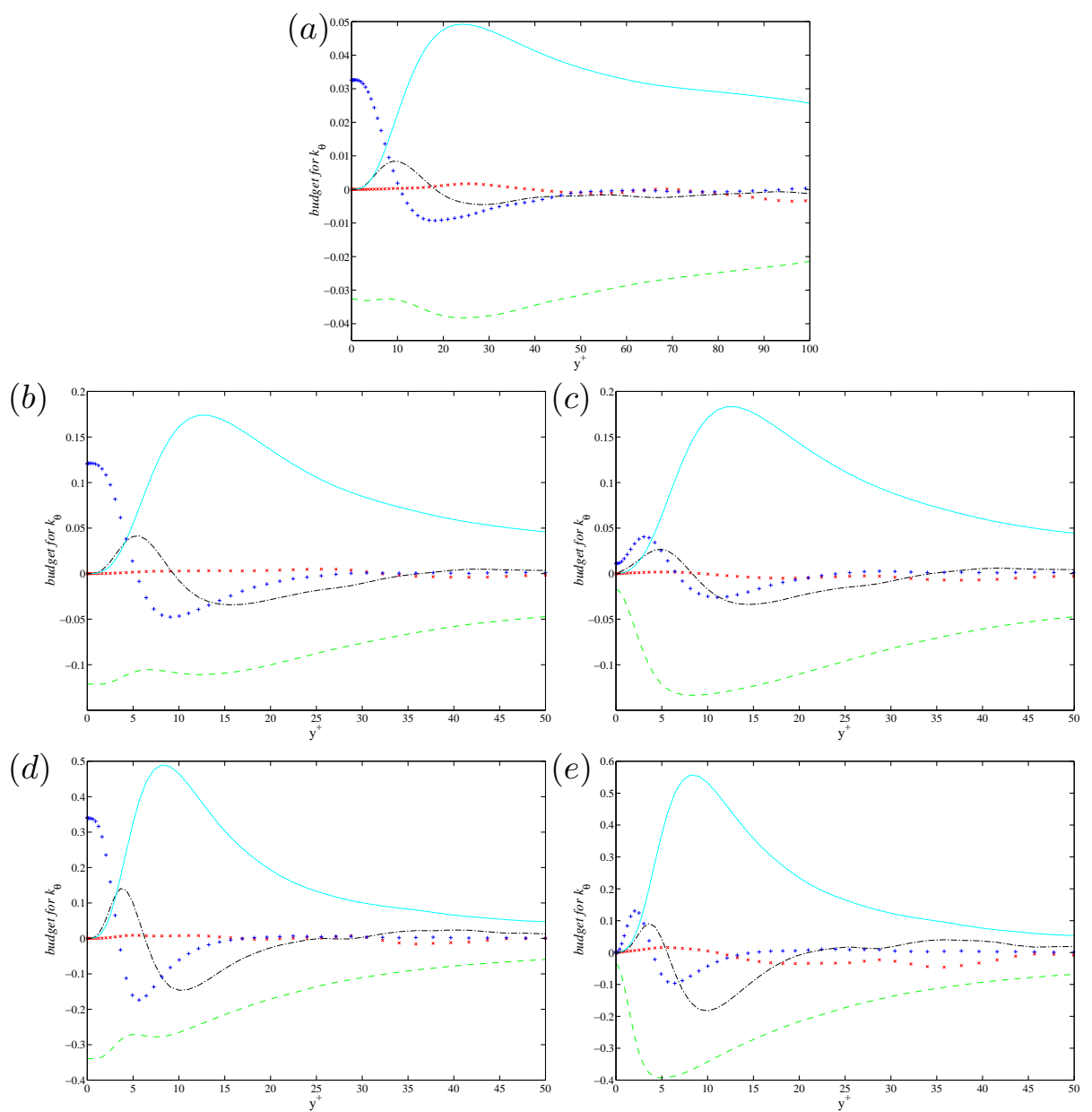

Figure 4.44: Budget for the fluctuating scalar variance at $R e_{\theta}=830$.

- Production, - - -Dissipation, -.-Turbulent diffusion, ........ Velocity pressure diffusion, + Molecular diffusion, * Mean convection. (a) $\theta_{1}$, (b) $\theta_{2},(c) \theta_{3},(d) \theta_{4},(e) \theta_{5}$.

the dissipation is negligible because of the isotropy in the dissipation scale. From the present DNS, it has actually been seen that the dissipation is negligibly small for $\operatorname{Pr}=2.0$ except in the vicinity of the wall. Thus, the production is balanced mainly by the scalar pressure-gradient correlation term. According to Kawamura et al. (1998), in a low Prandtl number fluid, the dissipation is dominant because it takes place in eddies of a larger scale. They reported that the scalar pressure-gradient term is dominant for $\mathrm{Pr}=$ 4.0 and 5.0 while the dissipation term is overwhelming for $\operatorname{Pr}=0.05$. They also reported that the scalar pressure-gradient and the dissipation terms become comparable at $\operatorname{Pr}=0.2$ which is not true for the present DNS. This might be due to the different types of flow. The spanwise scalar flux budget is not shown here since each term in the budget is small and just fluctuating 


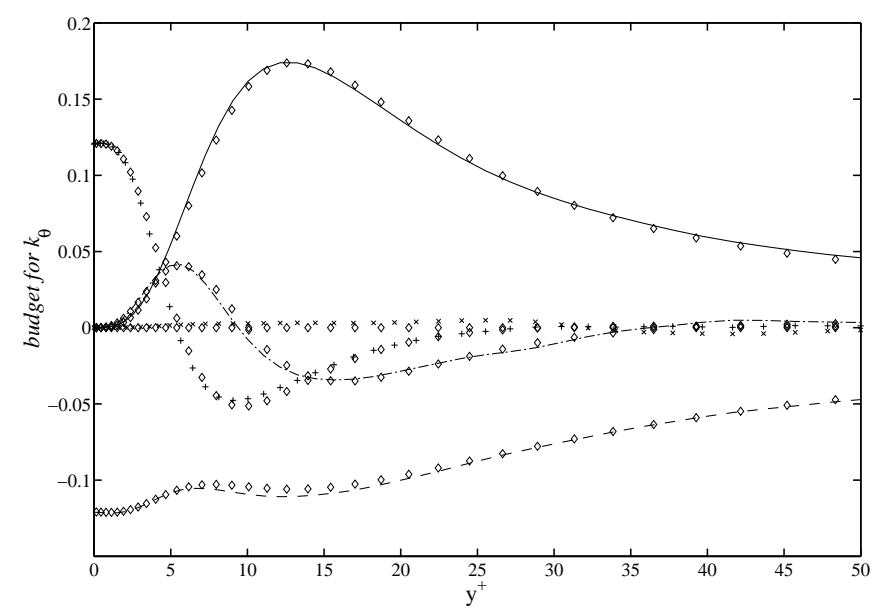

Figure 4.45: Comparison of the budget of the fluctuating scalar variance with other DNS data. — Production, - - - Dissipation, -- - Turbulent diffusion, ….... Velocity pressure diffusion, + Molecular diffusion, $*$ Mean convection, $\diamond$ Kawamura et al. (1998).

around zero.

A comparison of the scalar fluxes for $\theta_{2}$ is displayed in Figure 4.48 with the channel DNS data of Kawamura et al. (1998). Very good agreement with the DNS data of Kawamura et al. (1998) can be seen in the near wall region for the streamwise component. For the wall-normal component, a larger but still insignificant difference is observed. This difference might be due to the Reynolds number difference and different flow types.

In addition, the budgets of the scalar fluxes $\left\langle u^{\prime} \theta^{\prime}\right\rangle$ and $\left\langle v^{\prime} \theta^{\prime}\right\rangle$ look very much like those of the Reynolds stresses $\left\langle u^{\prime} u^{\prime}\right\rangle$ and $\left\langle u^{\prime} v^{\prime}\right\rangle$, respectively for $\operatorname{Pr}=0.71$. But this is not true for the present small Prandtl number case, i.e. $\operatorname{Pr}=0.2$. At even lower Prandtl number, see e.g. Kasagi and Ohtsubo (1993) with $\operatorname{Pr}=0.025$, the budgets profiles look even much more different.

\section{Pressure scalar-gradient correlation}

The budget equations for the streamwise and wall-normal scalar fluxes contain terms involving the scalar pressure-gradient correlation $C_{\theta i}$. Like the velocity pressure-gradient split, the scalar pressure-gradient term can also be split into a pressure-scalar diffusion term $G_{\theta i}$ and a pressure scalar-gradient term $\Pi_{\theta i}$ in the same way as follows

$$
C_{\theta i}=\Pi_{\theta i}+G_{\theta i} .
$$

These terms used in the scalar turbulence models are especially important. Among them, the pressure scalar-gradient term is the dominant one. The pressure scalar-gradient profiles versus the wall-normal positions at $R e_{\theta}=$ 830 are plotted in Figure 4.49. 

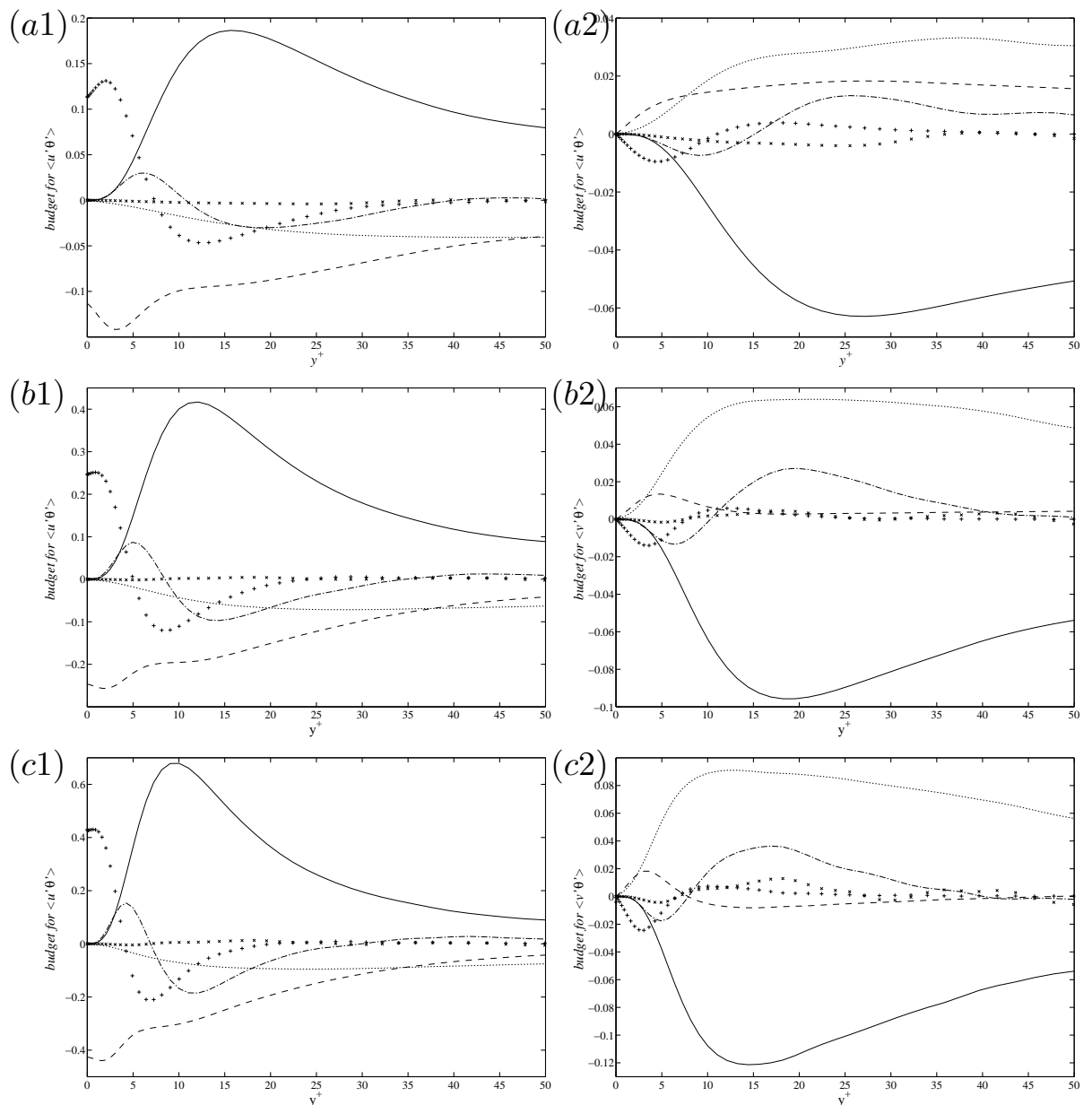

Figure 4.46: Budgets of the streamwise and wall-normal scalar flux fluctuations at $R e_{\theta}=830$ for isothermal boundary condition. - Production, - - - Dissipation, -.-Turbulent diffusion, ....... Scalar pressure diffusion, + Molecular diffusion, $*$ Mean convection. $(a) \theta_{1},(b) \theta_{2},(c) \theta_{4}$. (1) Streamwise scalar flux, (2) Wall-normal scalar flux.

Due to the wall boundary conditions, the wall-normal component at the wall is non-zero for the isothermal boundary condition and zero for the isoflux boundary condition. The streamwise and the spanwise components are opposite, i.e. zero at the wall for the isothermal boundary condition and non-zero for the isoflux boundary condition.

\subsubsection{Higher order statistics}

The skewness and flatness factors at different downstream positions are shown in Figures 4.50 and 4.51 for all the different scalars. Far away from the wall, neither the skewness factors nor the flatness factors are affected 

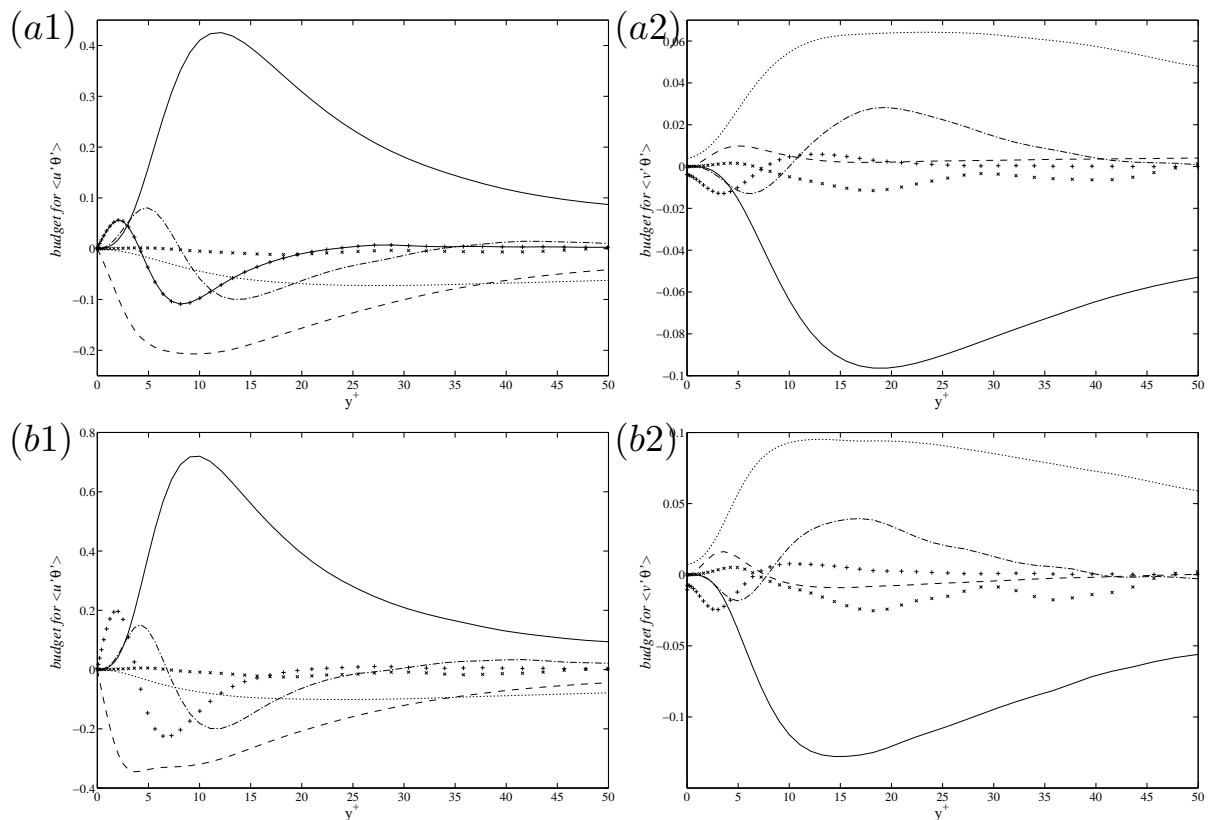

Figure 4.47: Budgets of the streamwise and wall-normal scalar flux fluctuations at $R e_{\theta}=830$ for isoflux boundary condition. - Production, - - - Dissipation, -.-Turbulent diffusion, ....... Scalar pressure diffusion, + Molecular diffusion, $*$ Mean convection. $(a) \theta_{3},(b) \theta_{5}$. (1) Streamwise scalar flux, (2) Wall-normal scalar flux.

(a)
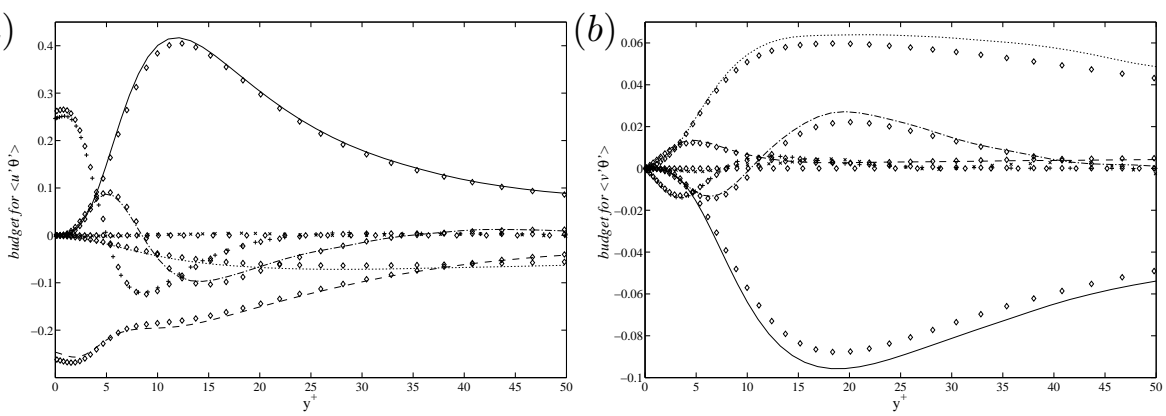

Figure 4.48: Comparison of the budget of the scalar fluxes with other DNS data. - Production, - - -Dissipation, -- Turbulent diffusion, ….... Velocity pressure diffusion, + Molecular diffusion, $*$ Mean convection, $\diamond$ Kawamura et al. (1998). (a) $\left\langle u^{\prime} \theta^{\prime}\right\rangle,(b)\left\langle v^{\prime} \theta^{\prime}\right\rangle$.

by the wall boundary conditions. Near the wall, $S(\theta)$ behave differently for different wall boundary conditions. For the isoflux wall, the $S(\theta)$ are closer to 0 which indicates that the scalar fluctuations are more symmetric in the vicinity of the wall than those of the isothermal wall. For the isothermal wall, within the sub-layer, $S(\theta)$ are positive which is consistent with the positive values of $S(u)$ and also reported by Antonia and Danh (1977). At 

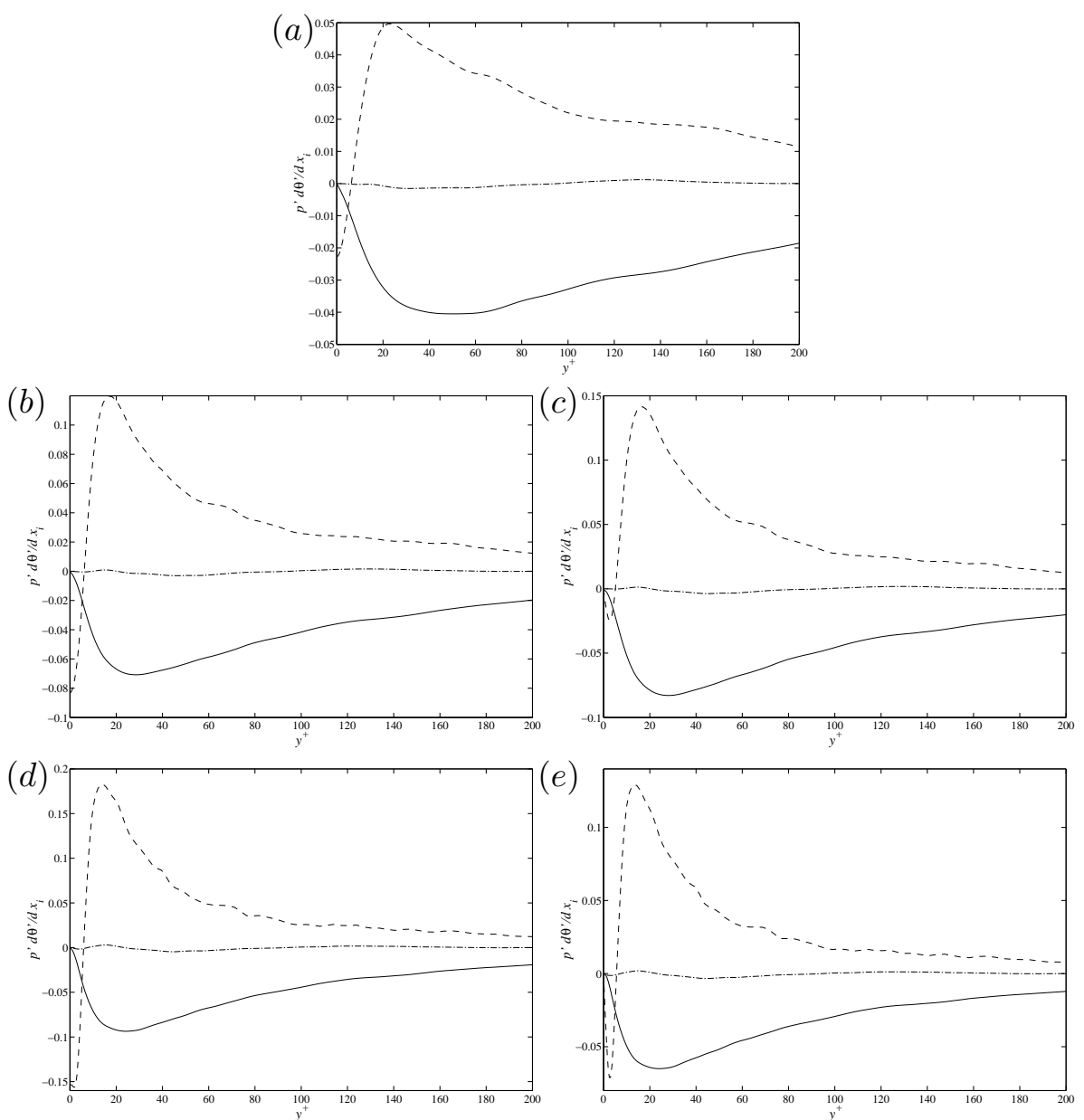

Figure 4.49: Pressure scalar-gradient correlation terms versus the wallnormal distance at $R e_{\theta}=830$. - Streamwise component, - - Wallnormal component, ---Spanwise component. (a) $\theta_{1}$, (b) $\theta_{2}$, (c) $\theta_{3},(d) \theta_{4}$, (e) $\theta_{5}$.

$y^{+} \approx 20, S\left(\theta_{2}\right)$ changes the sign. This wall-normal position is about twice larger than the one for the streamwise velocity and becomes larger as $\mathrm{Pr}$ decreases. This might be interpreted as that the scalar sub-layer thickness becomes thicker for smaller $\mathrm{Pr}$.

The flatness factors, $F(\theta)$, in the case of the isoflux wall, are almost constant and closer to 3 near the wall. As in the case of an isothermal boundary condition, $F(\theta)$ increase above 3 as the wall is approached. Further away from the wall, at $y^{+} \approx 18, F\left(\theta_{2}\right)$ appears to reach its lowest value around 2.3 which is not so distinct as that exhibited by $F(u)$. At larger wall-normal positions, unlike the behaviour of $F(u), F\left(\theta_{2}\right)$ increases slower towards 3 . One interesting thing is that the wall values of both the $S(\theta)$ and $F(\theta)$ for the isothermal boundary condition seem to be Prandtl number independent. 
In the outer region, the skewness and flatness factors for both isothermal and isoflux wall increase rapidly which indicates the existence of the intermittent region similarly as for the velocity and pressure. The maximum peak values of $S(\theta)$ and $F(\theta)$ are much higher than those of the $S(u)$ and $F(u)$ which is also observed in experiments by Antonia and Danh (1977). Outside the boundary layer, both $S(\theta)$ and $F(\theta)$ decrease rapidly toward the values of 0 and 3 , respectively.

Up to $y^{+}=100$, as the Reynolds number increases, it seems that the skewness and flatness factors increase only slightly for the isothermal boundary condition. However, for the isoflux boundary condition, the situation is opposite. Both the skewness and flatness factors are much more affected by the Reynolds number. A comparison of the skewness and flatness factors
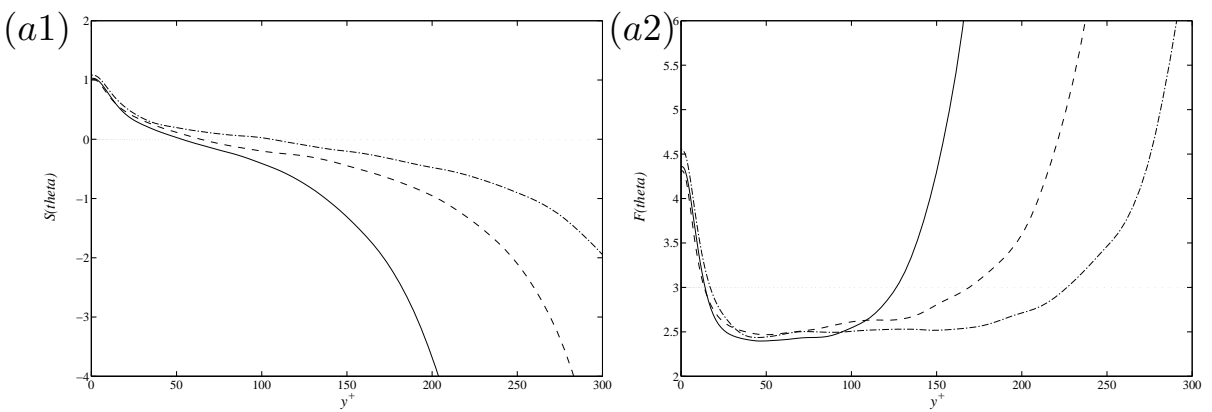

(b1)
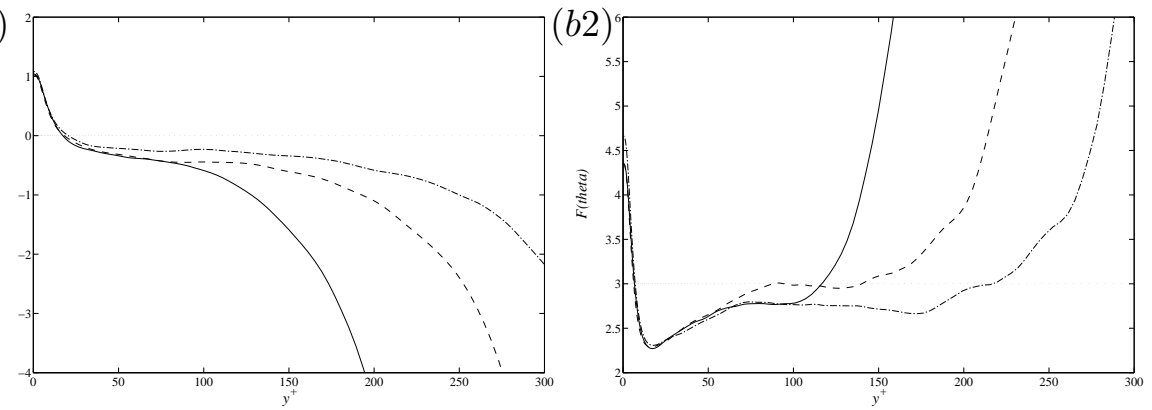

$(c 1)$
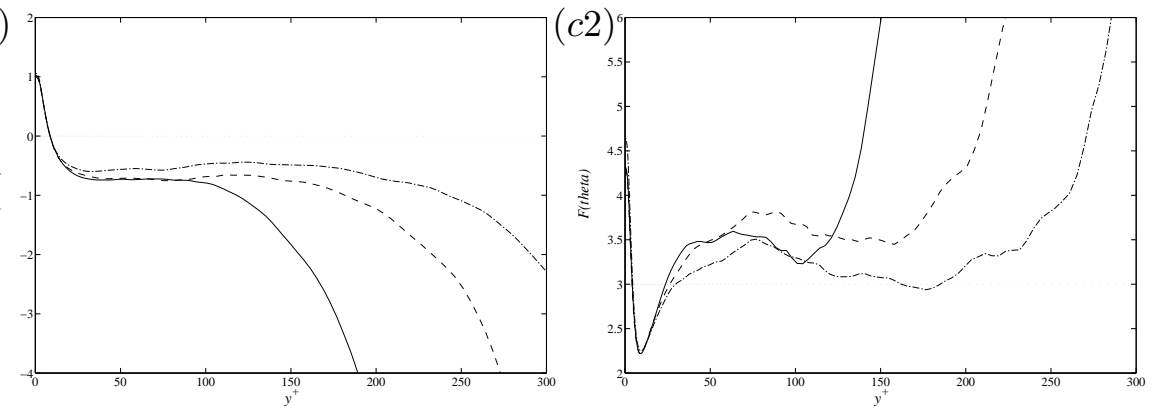

Figure 4.50: Variation of the skewness and flatness factors for isothermal boundary condition with downstream positions.—at $R e_{\theta}=396,-\mathbf{-}$ at $R e_{\theta}=628,-$ - at $R e_{\theta}=830$. (a) $\theta_{1}$, (b) $\theta_{2}$, (c) $\theta_{4}$. (1) Skewness factors, (2) Flatness factors. 

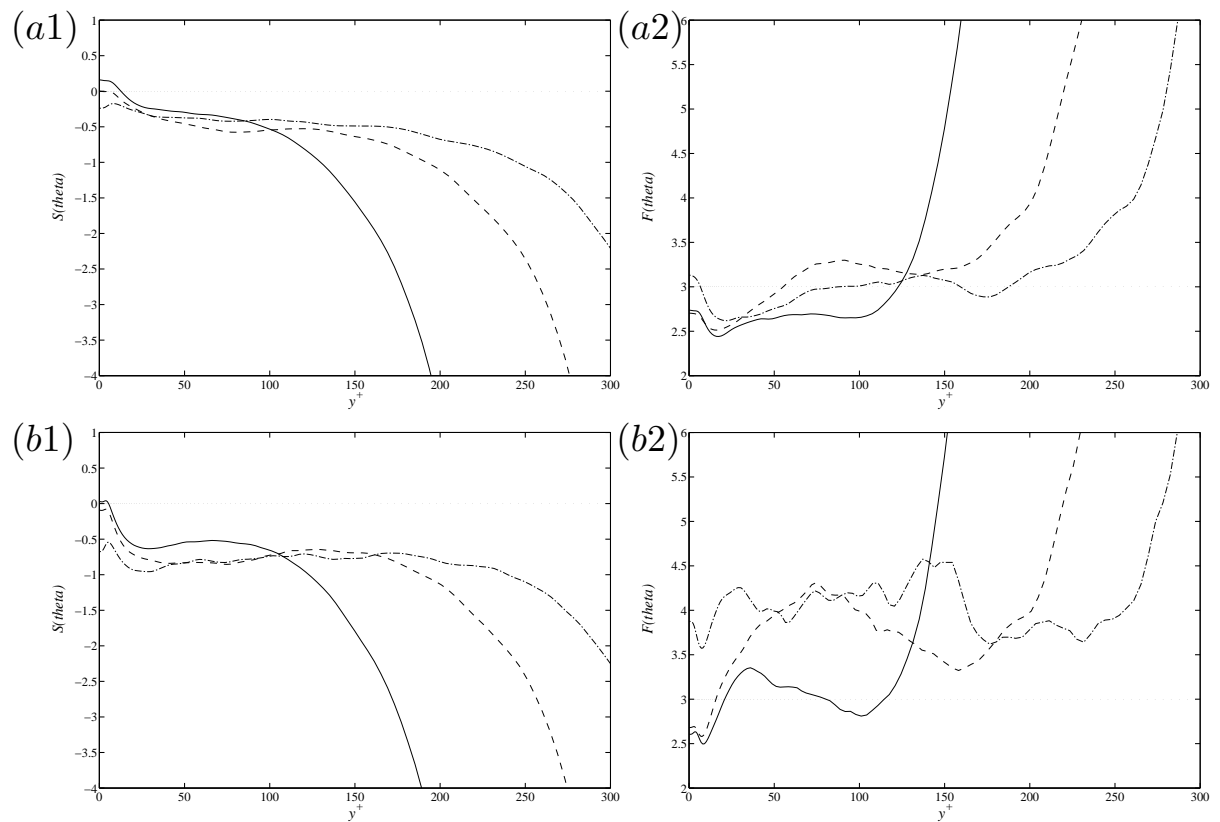

Figure 4.51: Variation of the skewness and flatness factors for isoflux boundary condition with downstream positions.—at $R e_{\theta}=396$, - - - at $R e_{\theta}=628,-$ at $R e_{\theta}=830$. (a) $\theta_{3}$, (b) $\theta_{5}$. (1) Skewness factors, (2) Flatness factors.

of $\theta_{2}$ at $R e_{\theta}=628$ with the channel DNS data from Kasagi et al. (1992) of $\operatorname{Pr}=0.71$ at $R e_{\tau}=150$ is shown in Figure 4.52. The agreement is very good in the near wall region, but poor far from the wall due to different types of flow.

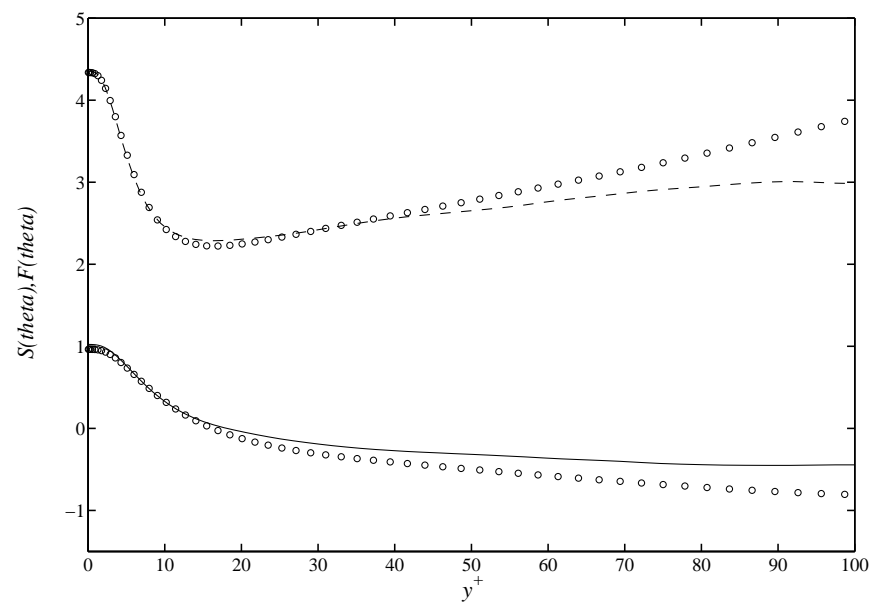

Figure 4.52: Comparison of skewness and flatness factor of $\theta_{2}$ at $R_{\theta}=628$ with other DNS data. $-S\left(\theta_{2}\right),---F\left(\theta_{2}\right)$, ○ Kasagi et al. (1992) at $R e_{\tau}=150$. 


\subsubsection{Turbulent structure of the scalar fields}

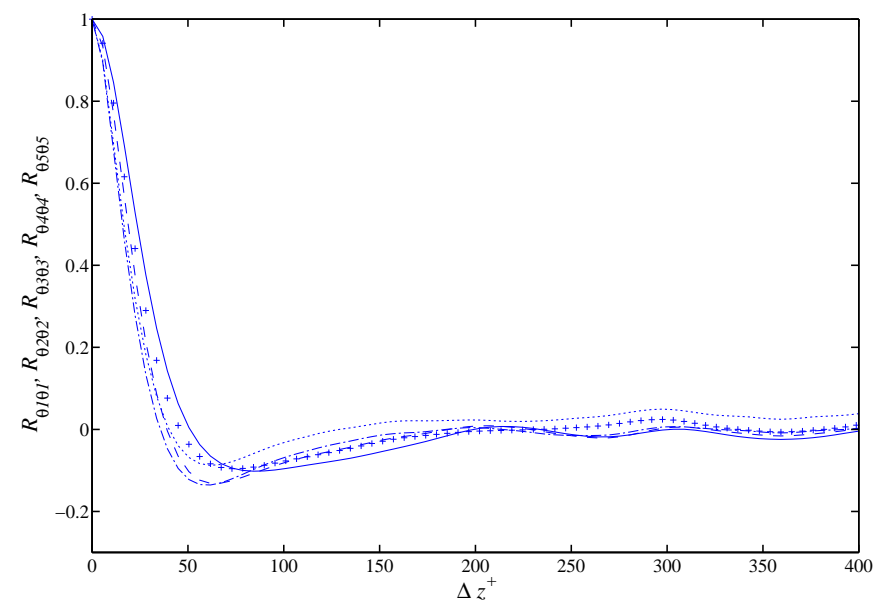

Figure 4.53: Spanwise two-point correlation coefficients of the scalar fluctuations at $y^{+}=9.8$ with $R_{\theta}=830 .-R_{\theta_{1} \theta_{1}},---R_{\theta_{2} \theta_{2}},+R_{\theta_{3} \theta_{3}}$, $-\cdot-R_{\theta_{4} \theta_{4}}, \cdots \cdots \cdot R_{\theta_{5} \theta_{5}}$.

Kim and Moin (1989) reported that the correlation coefficient between the streamwise velocity and the scalar of $\operatorname{Pr}=0.71$ and 2.0 is as high as 0.95 in the wall region. Due to this high correlation between the streamwise velocity and the scalar, we expect that the mean spanwise spacing of the thermal streaks is about 100 in wall units. By examining the spanwise twopoint correlation of the scalar fluctuations as in the case of the streamwise velocity, we find that the mean spanwise spacing of the scalar streaks is more or less as we expected.

The spanwise two-point correlations of the different scalars at $y^{+}=10$ are shown in Figure 4.53. From the plot, we can see that at this wall-normal position, even though there is a large difference between the Prandtl numbers of $\theta_{2}$ and $\theta_{4}$ of the isothermal boundary condition, almost no difference between $R_{\theta_{2} \theta_{2}}$ and $R_{\theta_{4} \theta_{4}}$ exists. In contrast to the mean scalar profiles which are strongly affected by the $\operatorname{Pr}$, see Figure $4.30, R_{\theta \theta}$ for the isothermal boundary condition appears to be independent of the molecular Prandtl number. However, an obvious deviation of $R_{\theta_{1} \theta_{1}}$ from $R_{\theta_{2} \theta_{2}}$ and $R_{\theta_{4} \theta_{4}}$ indicates that this can not be true when the Prandtl number is too small (Kim and Moin, 1989). On the contrary, the scalars of the isoflux boundary condition seem to be much more affected by the Prandtl number and have larger mean spanwise spacings than those of the isothermal boundary condition.

Figure 4.54 shows the dependence of the spanwise two-point correlation coefficients of the scalar fluctuations on downstream positions. In a similar way as the streamwise velocity fluctuation, the first minimum becomes less prominent as the Reynolds number increases. This might be due to the fact that the scalar streaks are clustered for a higher Reynolds number. 

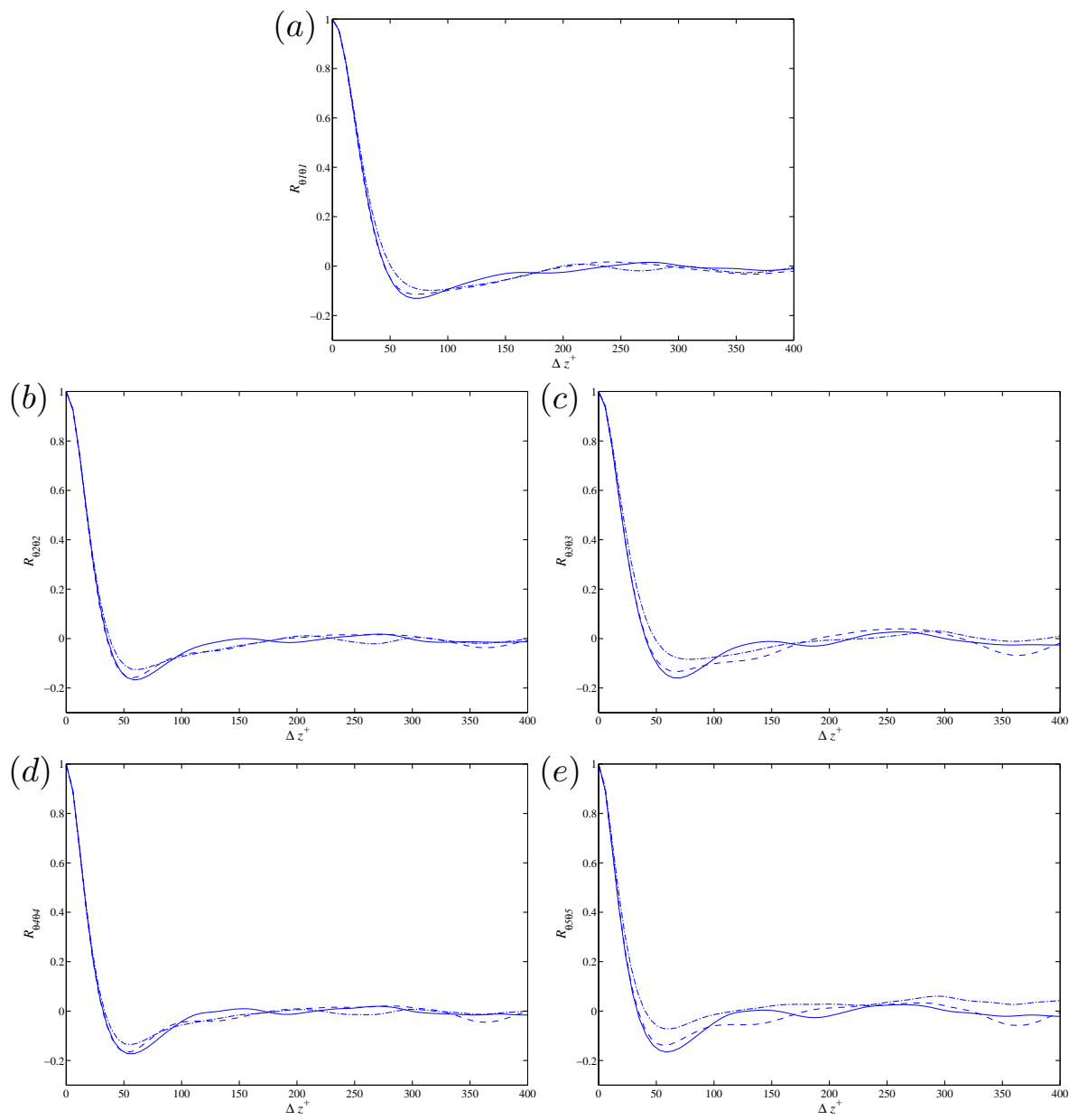

Figure 4.54: Spanwise two-point correlation coefficients of the scalar fluctuation with varying Reynolds numbers. - at $R e_{\theta}=628,---$ at $R e_{\theta}=728$, -. - at $R e_{\theta}=830$. (a) $\theta_{1},(b) \theta_{2},(c) \theta_{3},(d) \theta_{4},(e) \theta_{5}$.

Additionally as expected, the mean streak spacing is increasing as departing from the wall. Figure 4.55 shows the variation of the mean spanwise spacing of the scalar streaks at $R e_{\theta}=830$ versus the wall-normal distances $y^{+}$. The scalar streak spacings for the isothermal wall boundary condition are similar to that of the low-speed streaks, but are smaller than the those for the isoflux wall, especially in the near wall region.

\subsubsection{Probability density functions}

The PDF distributions of the different scalar fluctuations at various wallnormal positions, from $y^{+}=5$ in the conductive sub-layer to $y^{+}=300$ at the edge of the boundary layer, are shown in Figure 4.56 to 4.60. A Gaussian distribution is also shown as a reference denoted by the dashed 


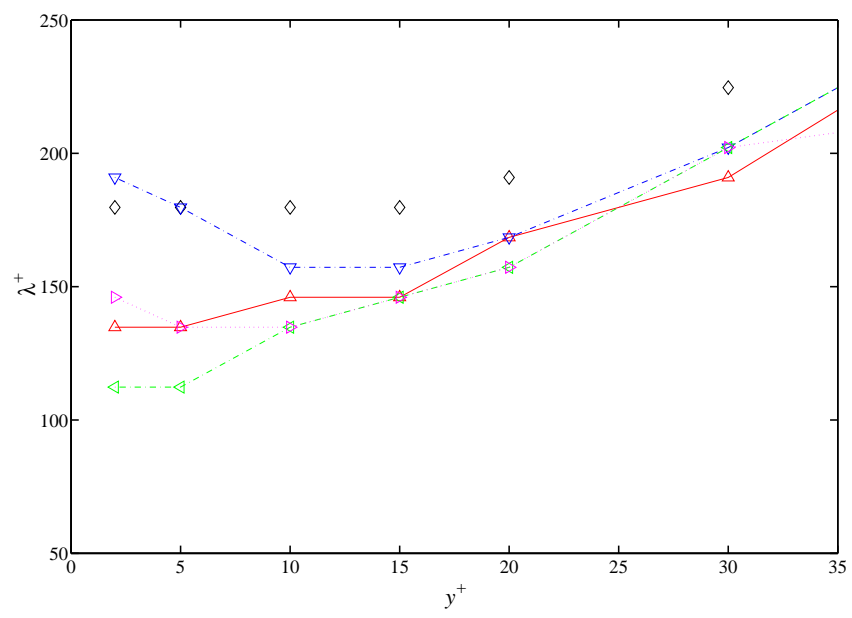

Figure 4.55: Variation of the mean spanwise scalar steaks spacing with varying wall-normal positions at $R_{\theta}=830 . \diamond \theta_{1}, \triangle \theta_{2}, \nabla \theta_{3}, \triangleleft \theta_{4}, \triangleright \theta_{5}$.

line. As what we did for the velocity and pressure fluctuations, the scalar fluctuations are also normalised by the corresponding RMS values, and the probability density distributions are normalised in such a way that the area under each curve is unity.

The PDF of the scalar fluctuations of the isothermal boundary condition are positively skewed in the near wall region, which can also been seen from the skewness factors $S(\theta)$, and successively changes to negatively skewed. A long negative tail at $y^{+} \approx 5$ also exists as the one for the streamwise velocity and this can be interpreted as being caused by the sweep-type motion. At larger wall-normal positions, $P\left(\theta^{\prime}\right)$ become symmetric, which are the same positions where $S(\theta)=0$. Very far away from the wall at $y^{+} \approx 300$, the PDF of the scalar fluctuations are extremely negatively skewed which indicates the existence of the intermittent region.

$P\left(\theta_{2}^{\prime}\right)$ compared with the channel DNS data from Abe et al. (2004) at $y^{+} \approx 10$ and $y^{+} \approx 200$ is plotted in Figure 4.61. Agreement with the channel DNS is good as seen from the plot.

By examining the PDF distributions of $\theta_{2}^{\prime}$ and $u^{\prime}$ at $y^{+}=1.92$ shown in Figures 4.24 and 4.60 , we found that the negative tail of the $P\left(\theta_{2}^{\prime}\right)$ does not extend as far as that for the $P\left(u^{\prime}\right)$. This is also reported by Zaric (1975) and Abe et al. (2004). According to Abe et al. (2004), this noticeable difference is closely associated with the large wall pressure fluctuation. And the importance of the pressure in causing the dissimilarity between the momentum and scalar fields has been reported by several researchers, e.g. Kong et al. (2001). 

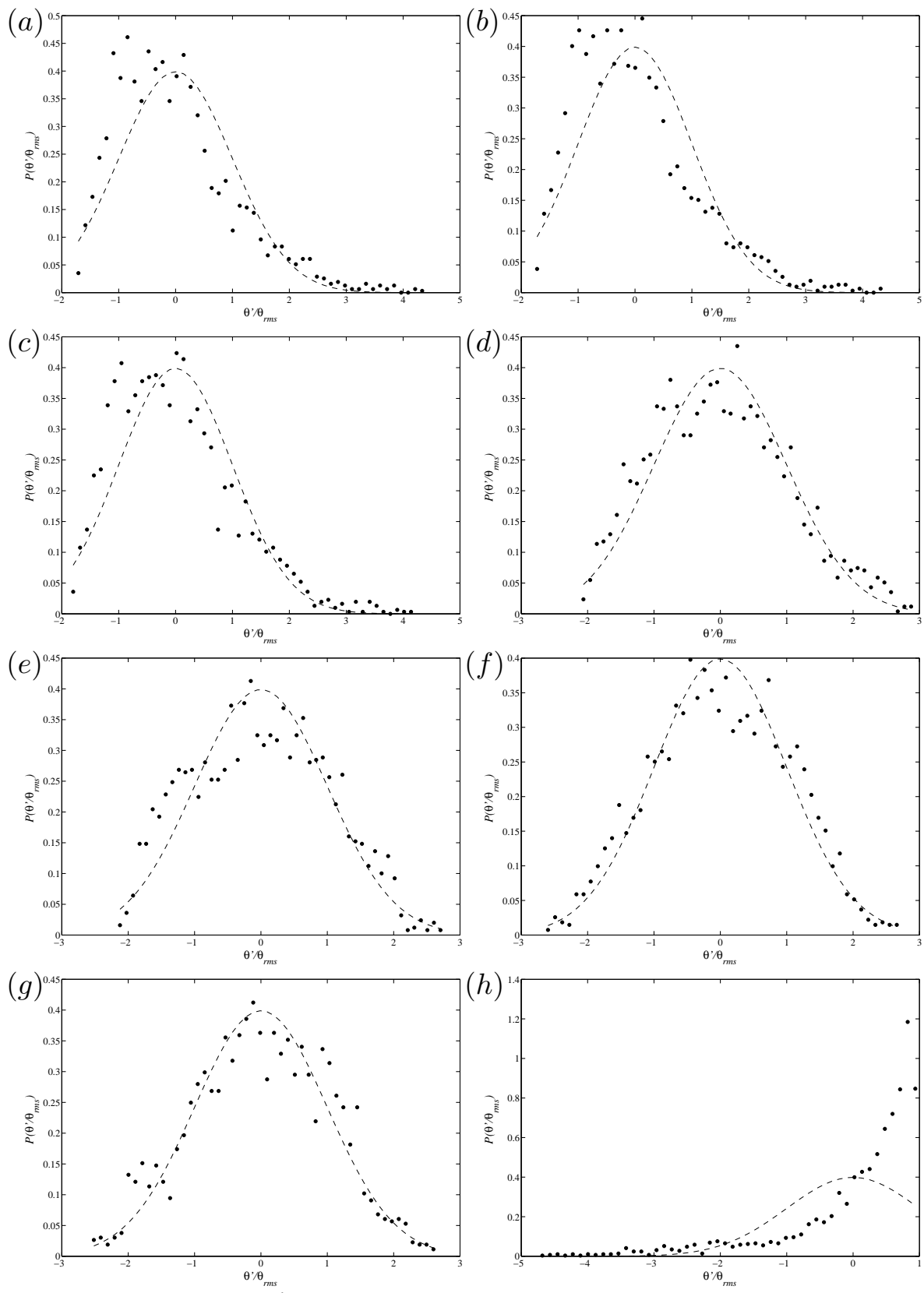

Figure 4.56: $\mathrm{PDF}$ of $\theta_{1}^{\prime}$ with $\operatorname{Pr}=0.2$ and isothermal wall and $\operatorname{Re}_{\theta}=830$. (a) $y^{+}=1.92, S(u)=1.08$, (b) $y^{+}=4.87, S(u)=1.02$, (c) $y^{+}=$ 9.74, $S(u)=0.85,(d) y^{+}=29.3, S(u)=0.37,(e) y^{+}=48.7, S(u)=0.20$, $(f) y^{+}=73.1, S(u)=0.10,(g) y^{+}=97.4, S(u)=0.03,(h) y^{+}=$ $292.3, S(u)=-1.72$.

\subsubsection{Quadrant analysis}

A quadrant analysis of the wall-normal scalar flux $\left\langle v^{\prime} \theta^{\prime}\right\rangle$ is performed in order to investigate the coherence between the velocity and scalar fluctuations 

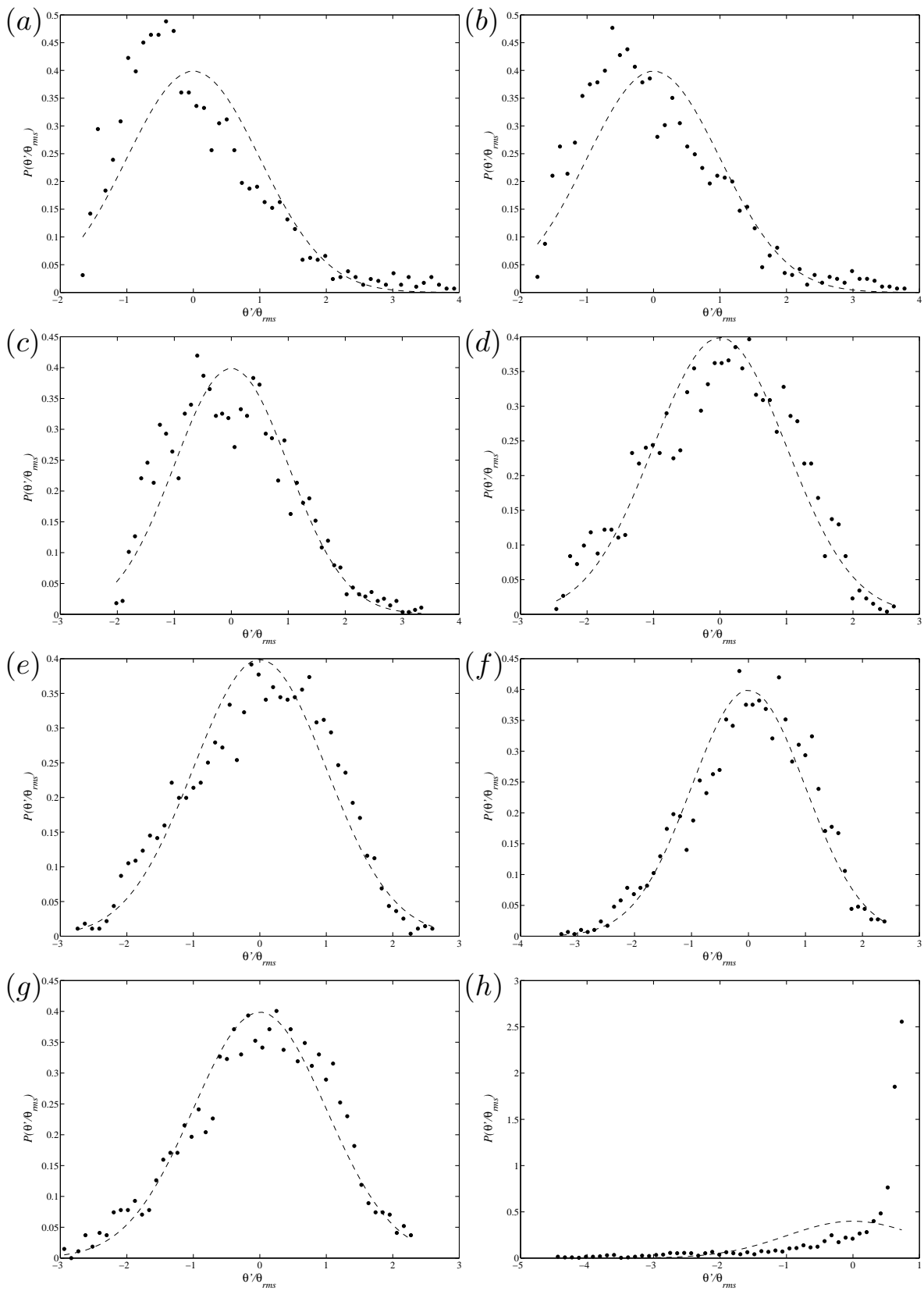

Figure 4.57: $\mathrm{PDF}$ of $\theta_{2}^{\prime}$ with $\operatorname{Pr}=0.71$ and isothermal wall and $R e_{\theta}=$ 830. (a) $y^{+}=1.92, S(u)=1.05,(b) y^{+}=4.87, S(u)=0.83$, (c) $y^{+}=$ 9.74, $S(u)=0.40,(d) y^{+}=29.3, S(u)=-0.13$, (e) $y^{+}=48.7, S(u)=$ $-0.21,(f) y^{+}=73.1, S(u)=-0.26,(g) y^{+}=97.4, S(u)=-0.23,(h)$ $y^{+}=292.3, S(u)=-1.92$.

and the results are shown in Figure 4.62. The present results are in good agreement with the simulation by Kong et al. (2000). 

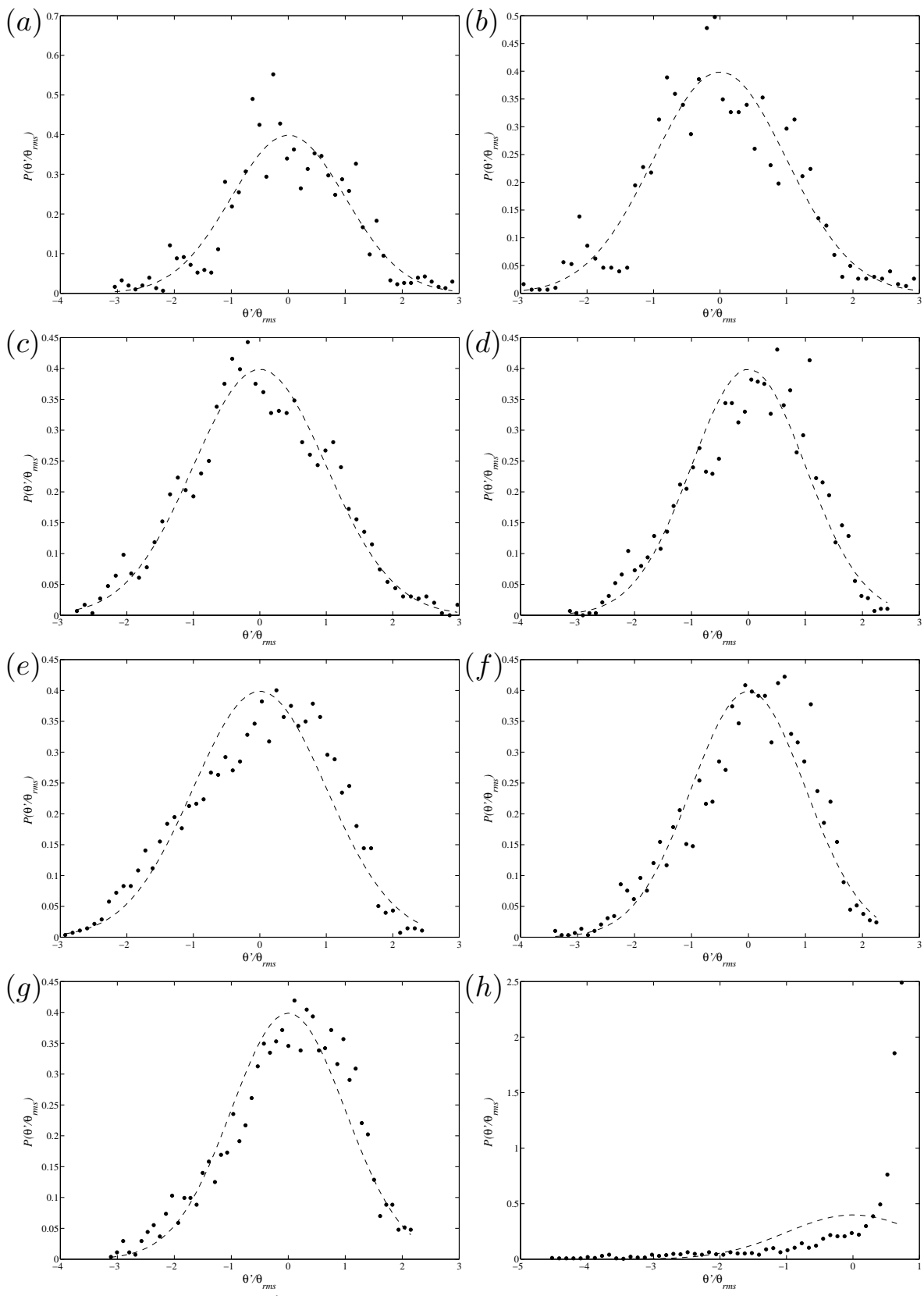

Figure 4.58: PDF of $\theta_{3}^{\prime}$ with $\operatorname{Pr}=0.71$ and isoflux wall and $\operatorname{Re}_{\theta}=830$. (a) $y^{+}=0, S(u)=-0.24,(b) y^{+}=4.87, S(u)=-0.20,(c) y^{+}=9.74, S(u)=$ $-0.18,(d) y^{+}=29.3, S(u)=-0.34,(e) y^{+}=48.7, S(u)=-0.37,(f) y^{+}=$ 73.1, $S(u)=-0.42,(g) y^{+}=97.4, S(u)=-0.40,(h) y^{+}=292.3, S(u)=$ -1.96 .

For the scalars with the isothermal boundary condition, the contributions to the wall-normal scalar flux are either from the ejection-type events in the 

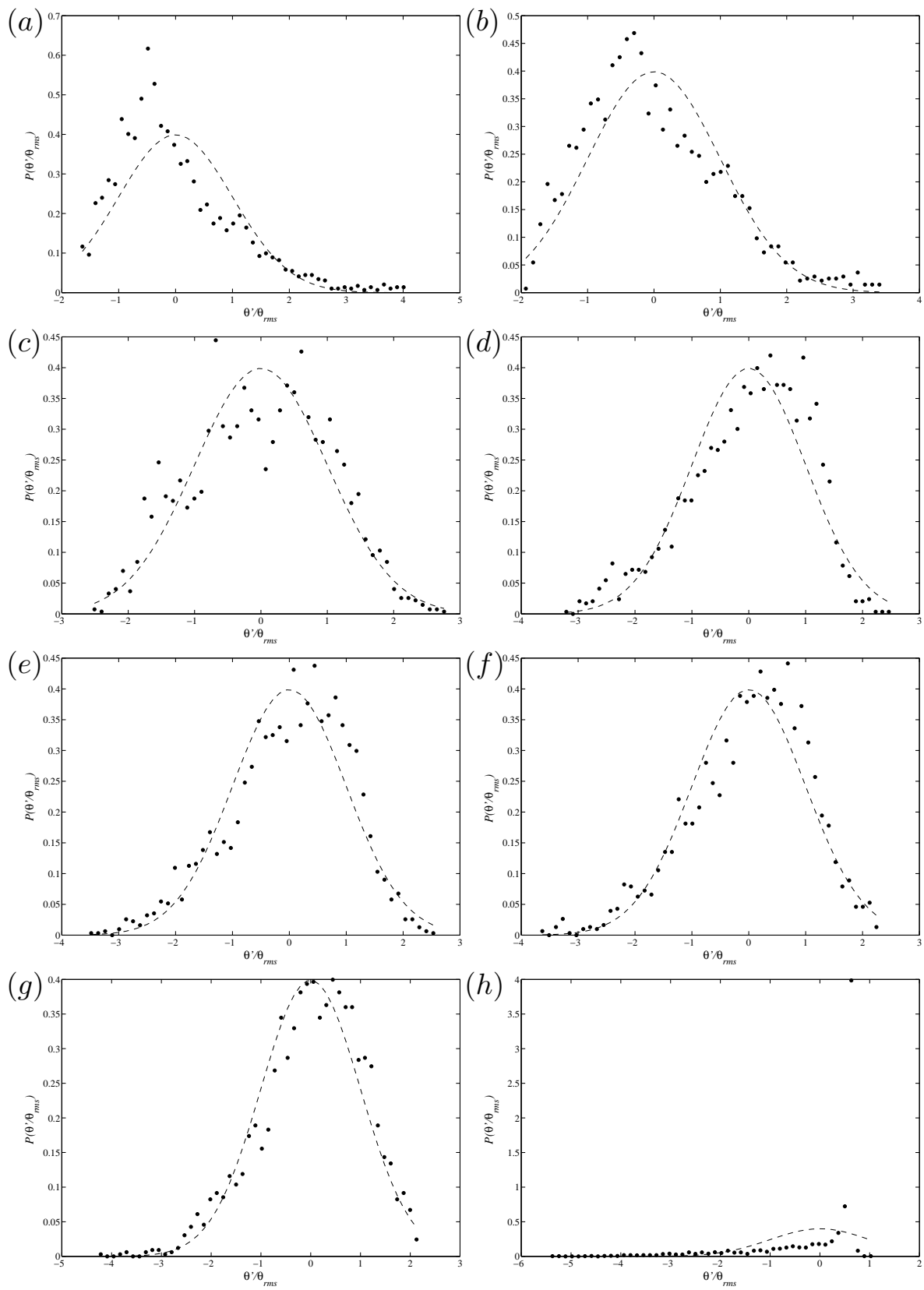

Figure 4.59: $\mathrm{PDF}$ of $\theta_{4}^{\prime}$ with $\operatorname{Pr}=2$ and isothermal wall and $\operatorname{Re}_{\theta}=830$. (a) $y^{+}=1.92, S(u)=0.99,(b) y^{+}=4.87, S(u)=0.53,(c) y^{+}=9.74, S(u)=$ $-0.10,(d) y^{+}=29.3, S(u)=-0.59,(e) y^{+}=48.7, S(u)=-0.57,(f) y^{+}=$ $73.1, S(u)=-0.58,(g) y^{+}=97.4, S(u)=-0.48,(h) y^{+}=292.3, S(u)=$ -2.04 .

second quadrant, i.e. $\theta<0$ and $v^{\prime}>0$, or the sweep-type events in the fourth quadrant, i.e. $\theta>0$ and $v^{\prime}<0$. This can be seen from the Figure 

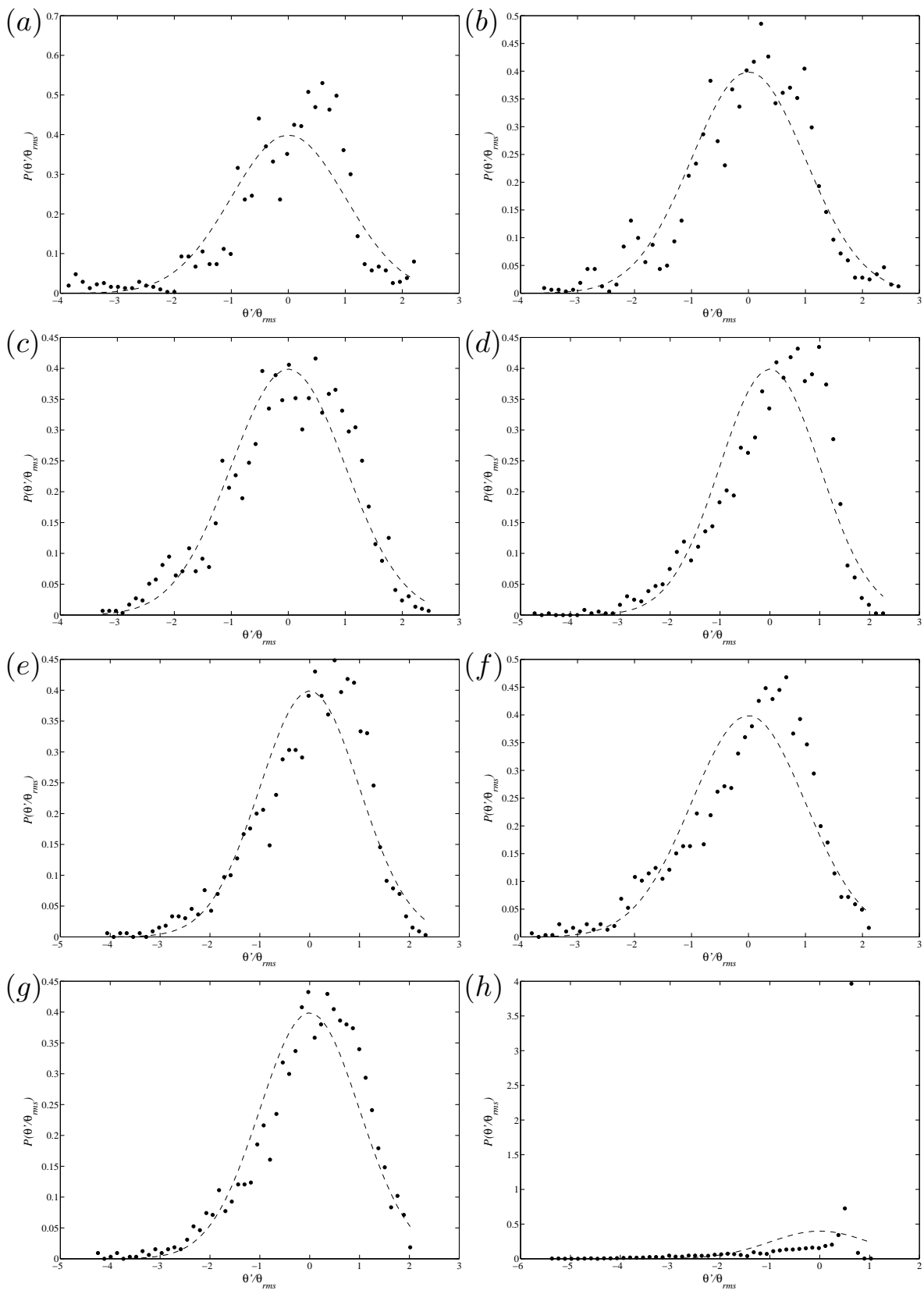

Figure 4.60: PDF of $\theta_{5}^{\prime}$ with $\operatorname{Pr}=2$ and isoflux wall and $R e_{\theta}=830$. (a) $y^{+}=0, S(u)=-0.68,(b) y^{+}=4.87, S(u)=-0.55,(c) y^{+}=9.74, S(u)=$ $-0.68,(d) y^{+}=29.3, S(u)=-0.96,(e) y^{+}=48.7, S(u)=-0.84,(f) y^{+}=$ 73.1, $S(u)=-0.83,(g) y^{+}=97.4, S(u)=-0.75$, (h) $y^{+}=292.3, S(u)=$ -2.00 .

4.62 which shows the contribution to the wall-normal scalar flux from each quadrant as a function of the non-dimensional wall-normal distance. The 

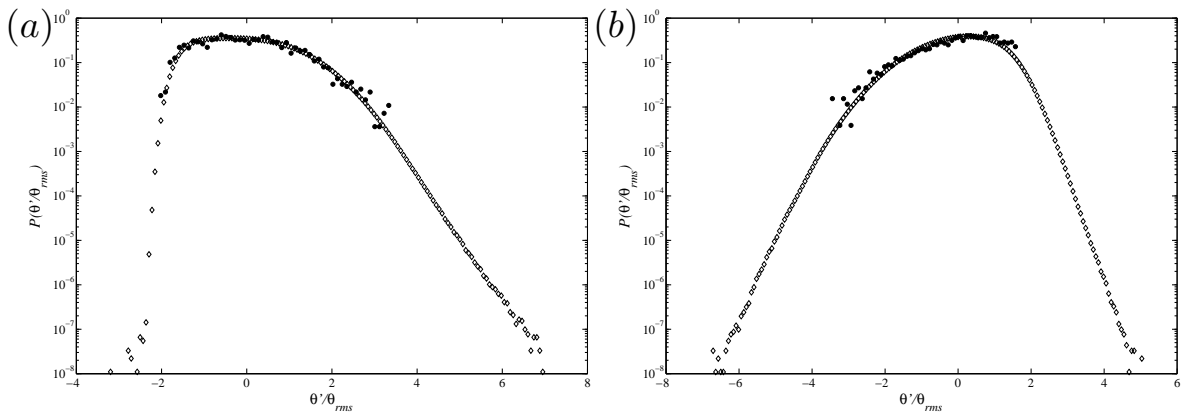

Figure 4.61: Comparison of the PDF of $\theta_{2}^{\prime}$ with other DNS data. • Present DNS at $R e_{\theta}=830, \diamond$ Abe et al. (2004) at $R e_{\tau}=395$. (a) $y^{+} \approx 10,(b)$ $y^{+} \approx 200$.
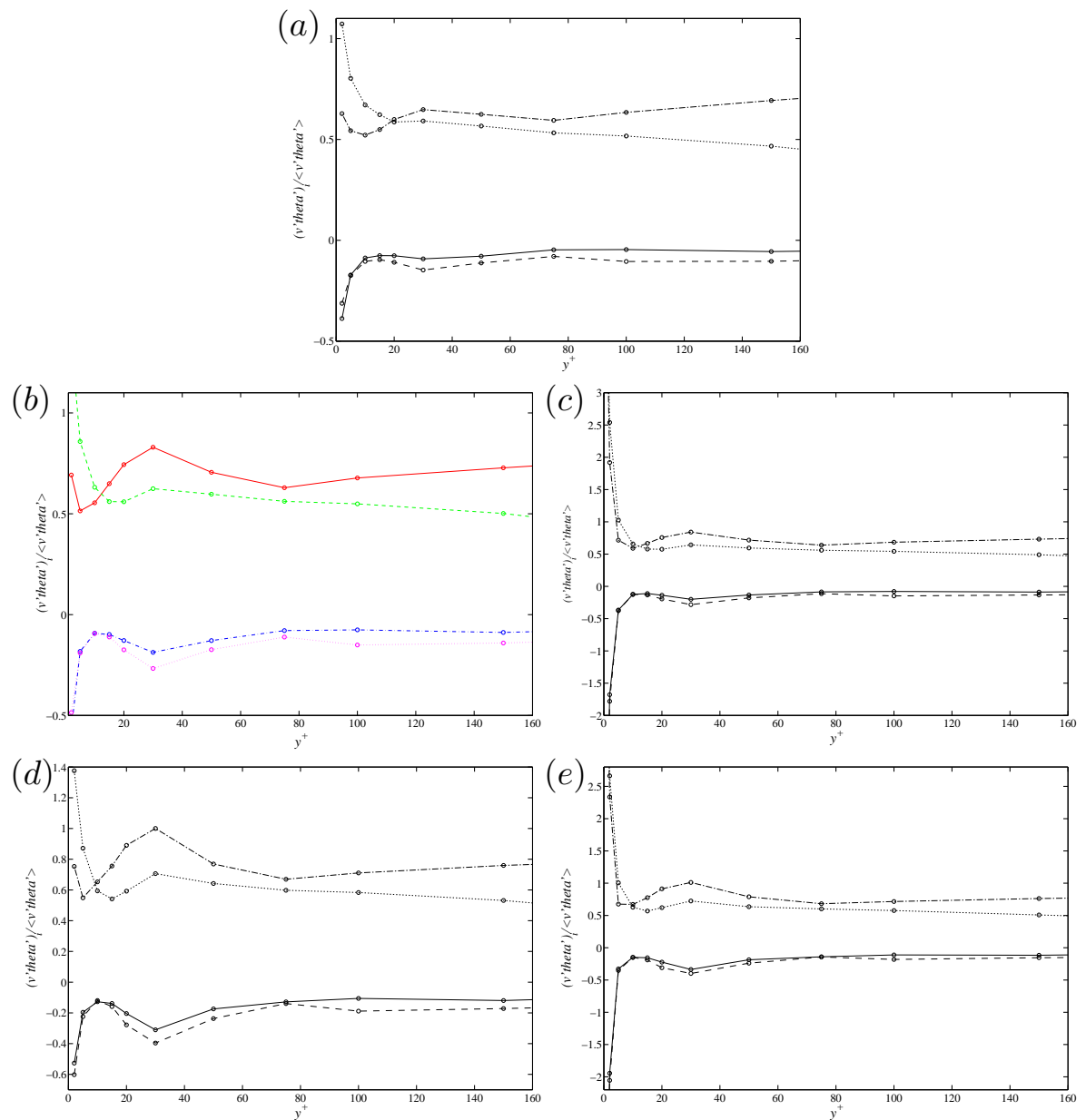

Figure 4.62: Fractional contribution to the wall-normal scalar flux $\left\langle v^{\prime} \theta^{\prime}\right\rangle$.

Ejection motion, - - -Sweep motion, -.-outward motion, ........ Wallward motion.(a) $v^{\prime} \theta_{1}^{\prime},(b) v^{\prime} \theta_{2}^{\prime},(c) v^{\prime} \theta_{3}^{\prime},(d) v^{\prime} \theta_{4}^{\prime},(e) v^{\prime} \theta_{5}^{\prime}$. 
wall-normal scalar flux is normalised by the local mean scalar flux $\left\langle v^{\prime} \theta^{\prime}\right\rangle$. For $\theta_{2}$, the contribution from the sweep-like motion predominates in the near wall region $y^{+}<12$, while that from the ejection-like motion becomes largest in the region $y^{+}>12$. The contributions from the other two types of motion, namely outward interaction-type and wall-ward interaction-type, are approximately equal through the entire boundary layer. These features are essentially the same as seen in the contribution to the Reynolds shear stress $\left\langle u^{\prime} v^{\prime}\right\rangle$ shown in Figure 4.28. On the other hand, for the scalars with the isoflux boundary condition, the profiles of the contribution to $\left\langle v^{\prime} \theta^{\prime}\right\rangle$ are almost the same as those with the isothermal boundary condition in the region $y^{+}>10$. However, near the wall $y^{+}<10$, the absolute values of the contribution from each quadrant are higher than those with the isothermal wall boundary condition. It is notable that the contributions from the first and third quadrants are not negligible at $y^{+}<10$ in case of the isoflux boundary condition.

Fractional contributions from the four quadrants to the total wall-normal scalar flux above a certain threshold value $H$ at $y^{+}=50$ are shown in Figure 4.63. From the plot, one can recognise that the scalar transport processes are dominated by the large amplitude ejection-type and sweep-type motions. The contributions from the other two interaction-type motions are negligible for $H>2.5$. The present results compare well with the experimental results by Nagano and Tagawa (1988).

\subsection{Flow Field VS. Scalar Field}

In order to examine the relation between the streamwise velocity and the scalars further more, we consider the probability density function of the Reynolds stress and the wall-normal scalar flux. We also use the joint probability density function (JPDF) between velocity and scalar fluctuations to give the quantitative measures for the interconnection between the flow and scalar fields.

\subsubsection{PDF of the Reynolds stress and scalar flux}

The PDF of the Reynolds stress and the scalar flux at $y^{+} \approx 30$ are shown in Figure 4.64. The distribution of the $u^{\prime} v^{\prime}$ is found to be very similar to that reported by Willmarth and $\mathrm{Lu}$ (1972) at the same wall-normal position. The distribution also has very long tails for extreme values of $u^{\prime} v^{\prime}$ and a sharp

peak at $u^{\prime} v^{\prime}=0$. One exception is that the tail for $\frac{u^{\prime} v^{\prime}}{\left\langle u^{\prime} v^{\prime}\right\rangle}<0$ reported by Willmarth and $\mathrm{Lu}$ (1972) is vanishing rapidly while the one for the present case extends for larger values. According to Willmarth and $\mathrm{Lu}$ (1972), the long tail is a result of the spiky nature of the Reynolds stress contributions during bursting events. 

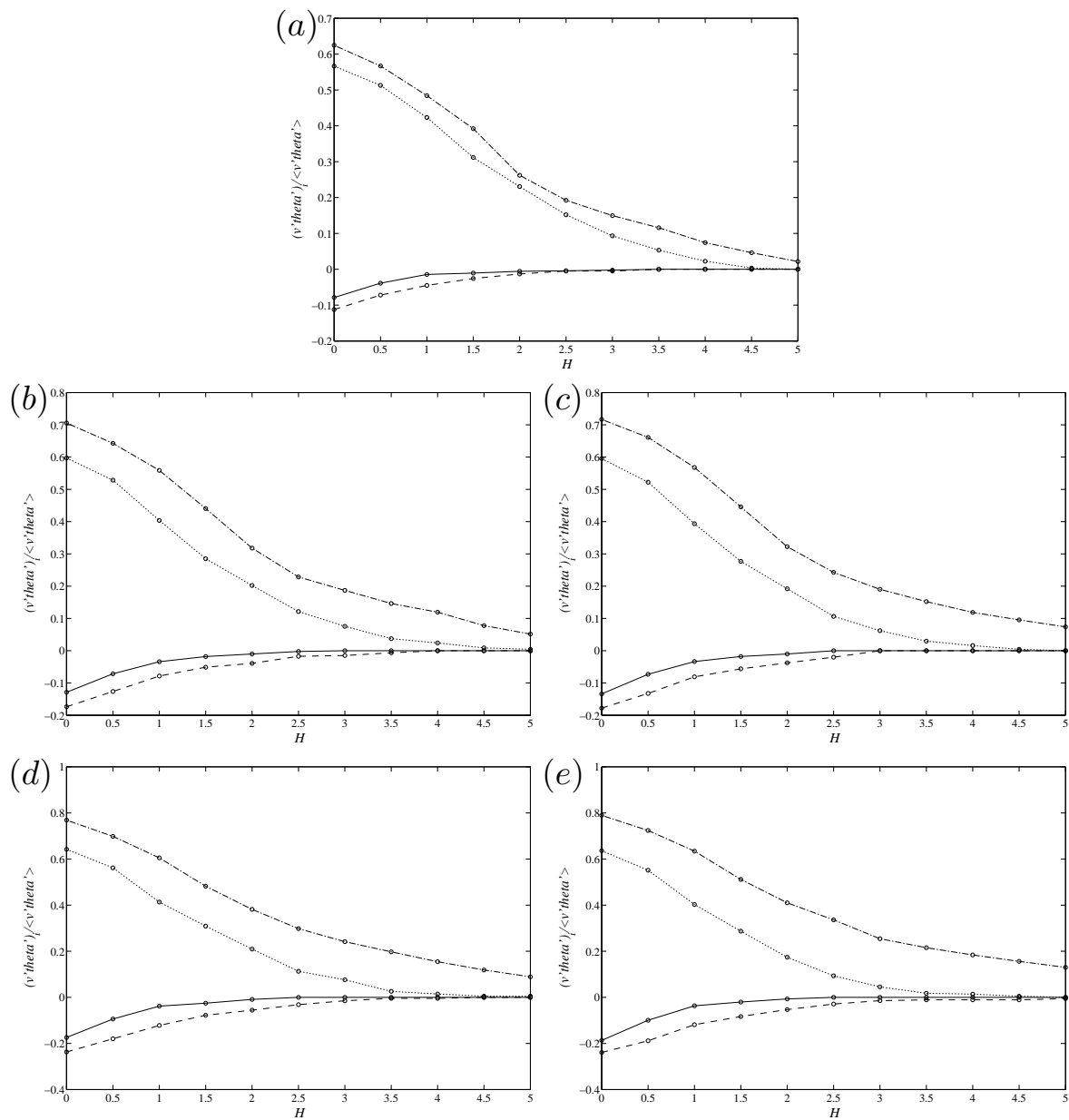

Figure 4.63: Fractional contribution to the wall-normal scalar flux $\left\langle v^{\prime} \theta^{\prime}\right\rangle$ as a function of threshold $H$. - Ejection motion, - - -Sweep motion, -.- outward motion, ........ Wall-ward motion. (a) $v^{\prime} \theta_{1}^{\prime}$, (b) $v^{\prime} \theta_{2}^{\prime},(c) v^{\prime} \theta_{3}^{\prime},(d)$ $v^{\prime} \theta_{4}^{\prime},(e) v^{\prime} \theta_{5}^{\prime}$.

In Figure $4.64(a)$, a Gaussian distribution of the $u^{\prime} v^{\prime}$ suggested by $\mathrm{Lu}$ and Willmarth (1973) is included for comparison. They assume that $u^{\prime}$ and $v^{\prime}$ are two statistically dependent random variables with correlation coefficient $R_{u v}=-0.44$, each obeying the Gaussian distribution. The joint distribution function of $u^{\prime}$ and $v^{\prime}$ is

$$
P\left(u_{1}, v_{1}\right)=\frac{1}{2\left(1-R_{u v}^{2}\right)^{\frac{1}{2}}} \exp \left(\frac{-1}{2\left(1-R_{u v}^{2}\right)}\left(u_{1}^{2}-2 R_{u v} u_{1} v_{1}+v_{1}^{2}\right)\right),
$$

where $u_{1}=\frac{u^{\prime}}{u_{r m s}}$ and $v_{1}=\frac{v^{\prime}}{v_{r m s}}$. After some transformations and integra- 

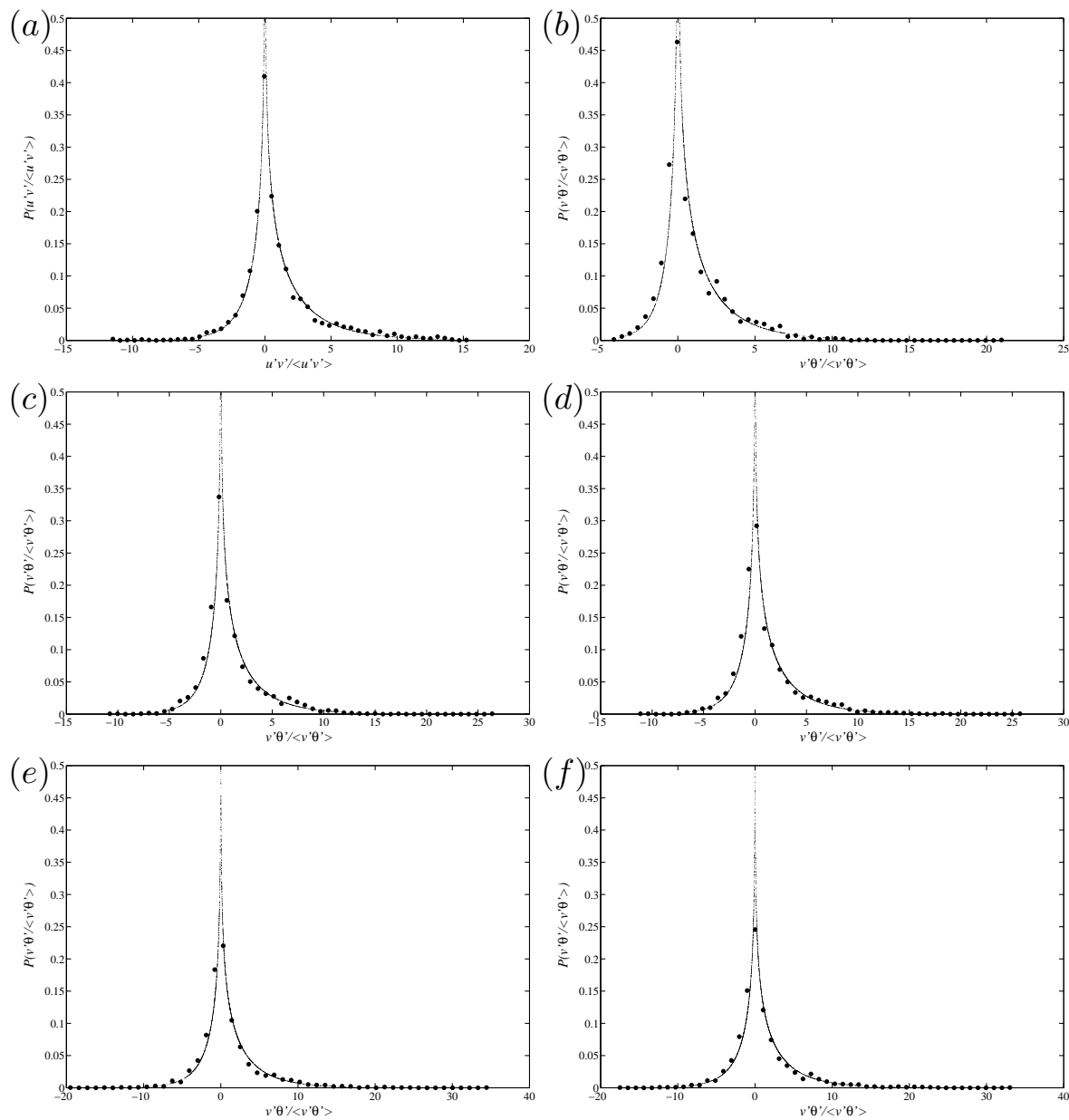

Figure 4.64: $\mathrm{PDF}$ of $u^{\prime} v^{\prime}$ and $v^{\prime} \theta^{\prime}$ at $y^{+}=29.3$ and $R e_{\theta}=830$. (a) $P\left(u^{\prime} v^{\prime}\right)$, (b) $P\left(v^{\prime} \theta_{1}^{\prime}\right)$, (c) $P\left(v^{\prime} \theta_{2}^{\prime}\right)$, (d) $P\left(v^{\prime} \theta_{3}^{\prime}\right)$, (e) $P\left(v^{\prime} \theta_{4}^{\prime}\right)$, (f) $P\left(v^{\prime} \theta_{5}^{\prime}\right)$.

tions, the probability density function for the normalised $\frac{u^{\prime} v^{\prime}}{\left\langle u^{\prime} v^{\prime}\right\rangle}$ reads

$$
P\left(\frac{u^{\prime} v^{\prime}}{\left\langle u^{\prime} v^{\prime}\right\rangle}\right)=\frac{R_{u v}}{\pi\left(1-R_{u v}^{2}\right)^{\frac{1}{2}}} \exp \left(\frac{R_{u v}^{2}\left\langle u^{\prime} v^{\prime}\right\rangle}{1-R_{u v}^{2}}\right) K_{0}\left(\left|\frac{R_{u v}\left\langle u^{\prime} v^{\prime}\right\rangle}{1-R_{u v}^{2}}\right|\right),
$$

where $K_{0}$ is the zeroth-order $K$ Bessel function. The agreement with the present DNS data appears to be satisfactory. Note that as $u^{\prime} v^{\prime} \rightarrow 0$, the Bessel function approaches infinity. Thus $P\left(\frac{u^{\prime} v^{\prime}}{\left\langle u^{\prime} v^{\prime}\right\rangle}\right) \rightarrow 0$ as $u^{\prime} v^{\prime} \rightarrow 0$. The distribution of $u^{\prime} v^{\prime}$ indicates the intermittent character of $u^{\prime} v^{\prime}$ near 0 since $u^{\prime} v^{\prime}$ will stay around 0 for most of the time which is also reported by $\mathrm{Lu}$ and Willmarth (1973).

The profiles of the PDF of the scalar fluxes are almost the same as the one for $u^{\prime} v^{\prime}$ which indicates a strong similarity between $u^{\prime}$ and $\theta^{\prime}$. The formula of the Gaussian distribution for the scalar fluxes is similar to equation 
(4.44). One interesting finding is that for the present DNS, even though the distributions of the velocity components and scalars do not obey the Gaussian distribution which can be seen from the PDF of each variable, the PDF of the Reynolds shear stress and the scalar fluxes still collapse with the equation (4.44).

\subsubsection{Joint PDF}
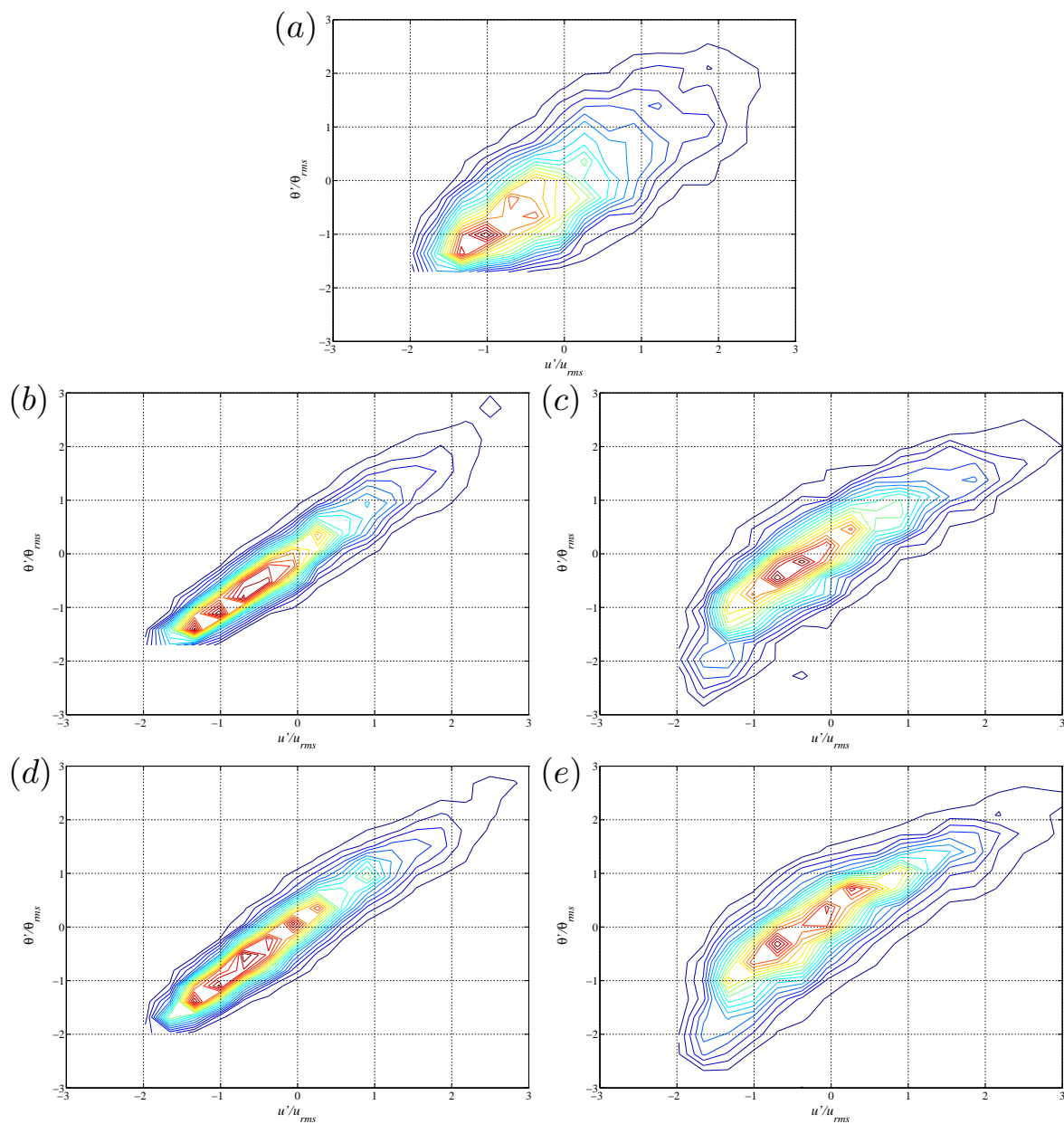

Figure 4.65: JPDF of $\left(u^{\prime}, \theta^{\prime}\right)$ at $y^{+} \approx 5$ and $R e_{\theta}=830$. (a) $P\left(u^{\prime}, \theta_{1}^{\prime}\right),(b)$ $P\left(u^{\prime}, \theta_{2}^{\prime}\right)$, (c) $P\left(u^{\prime}, \theta_{3}^{\prime}\right),(d) P\left(u^{\prime}, \theta_{4}^{\prime}\right),(e) P\left(u^{\prime}, \theta_{5}^{\prime}\right)$.

The high correlation between $u^{\prime}$ and $\theta^{\prime}$ can also be seen in Figure 4.65 and 4.66 where the JPDF of $\left(u^{\prime}, \theta^{\prime}\right),\left(u^{\prime}, v^{\prime}\right)$ and $\left(v^{\prime}, \theta^{\prime}\right)$ at $y^{+} \approx 5$ with $R e_{\theta}=830$ are shown. A strong positive correlation between $u^{\prime}$ and $\theta_{2}^{\prime}$ and a mild negative correlation between $v^{\prime}$ and $\theta_{2}^{\prime}$ are observed. These observations are essentially the same as those found by Kim (1988) and Kim and Moin (1989). However, for $\operatorname{Pr}=0.2, u^{\prime}$ is less correlated with $\theta_{1}$ due to the low $\operatorname{Pr}$ 

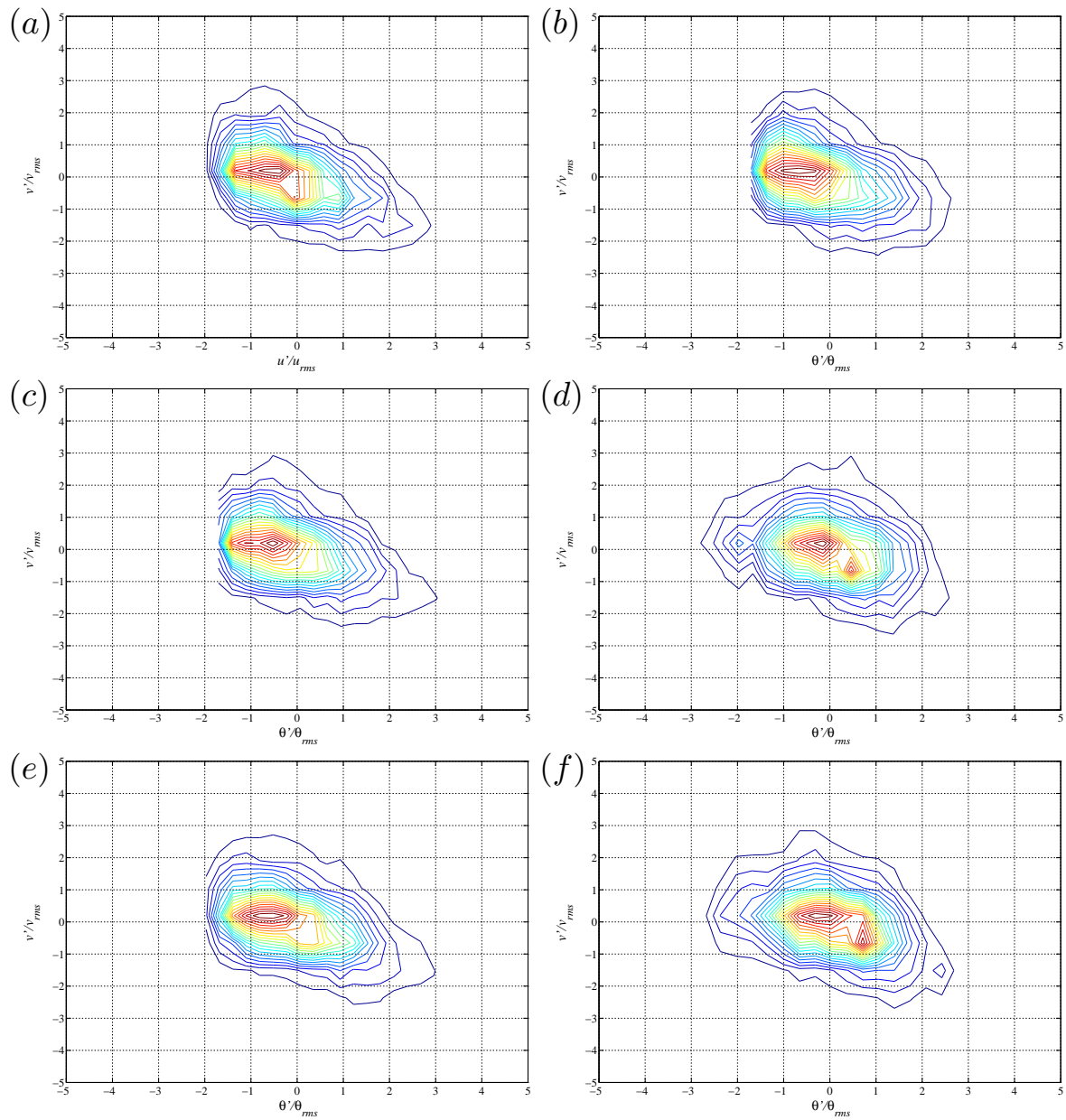

Figure 4.66: JPDF of $\left(u^{\prime}, v^{\prime}\right)$ and $\left(v^{\prime}, \theta^{\prime}\right)$ at $y^{+} \approx 5$ and $\operatorname{Re}_{\theta}=830$. (a) $P\left(u^{\prime}, v^{\prime}\right),(b) P\left(v^{\prime}, \theta_{1}^{\prime}\right),(c) P\left(v^{\prime}, \theta_{2}^{\prime}\right),(d) P\left(v^{\prime}, \theta_{3}^{\prime}\right)$, (e) $P\left(v^{\prime}, \theta_{4}^{\prime}\right),(f) P\left(v^{\prime}, \theta_{5}^{\prime}\right)$.

effect. Abe et al. (2004) also reported that for $\operatorname{Pr}=0.025$, the wall shear stress and the surface scalar flux are less correlated. We also found that less correlated relation between $u^{\prime}$ and $\theta_{3}$ and $\theta_{5}$. This is due to the isoflux boundary condition. Note that a perfect correlation will be represented by a straight line. Due to the high correlation between $u^{\prime}$ and $\theta_{2}^{\prime}$, the JPDF of $\left(v^{\prime}, \theta_{2}^{\prime}\right)$ looks very similar to that of $\left(u^{\prime}, v^{\prime}\right)$. Moreover, the JPDF of $\left(v^{\prime}, \theta_{1}^{\prime}\right)$ which is also very similar to the distribution of $\left(u^{\prime}, v^{\prime}\right)$, seems not affected by the low $\operatorname{Pr}$ effect. But the boundary condition still impose influences on the JPDF which can be seen from Figure $4.66(d)$ and $(f)$.

\subsubsection{Instantaneous fields}

Figure 4.67 shows the instantaneous streamwise velocity fluctuation at $y^{+} \approx$ 7 in the $(x, z)$ plane as well as the scalar fluctuations. Note that all the 

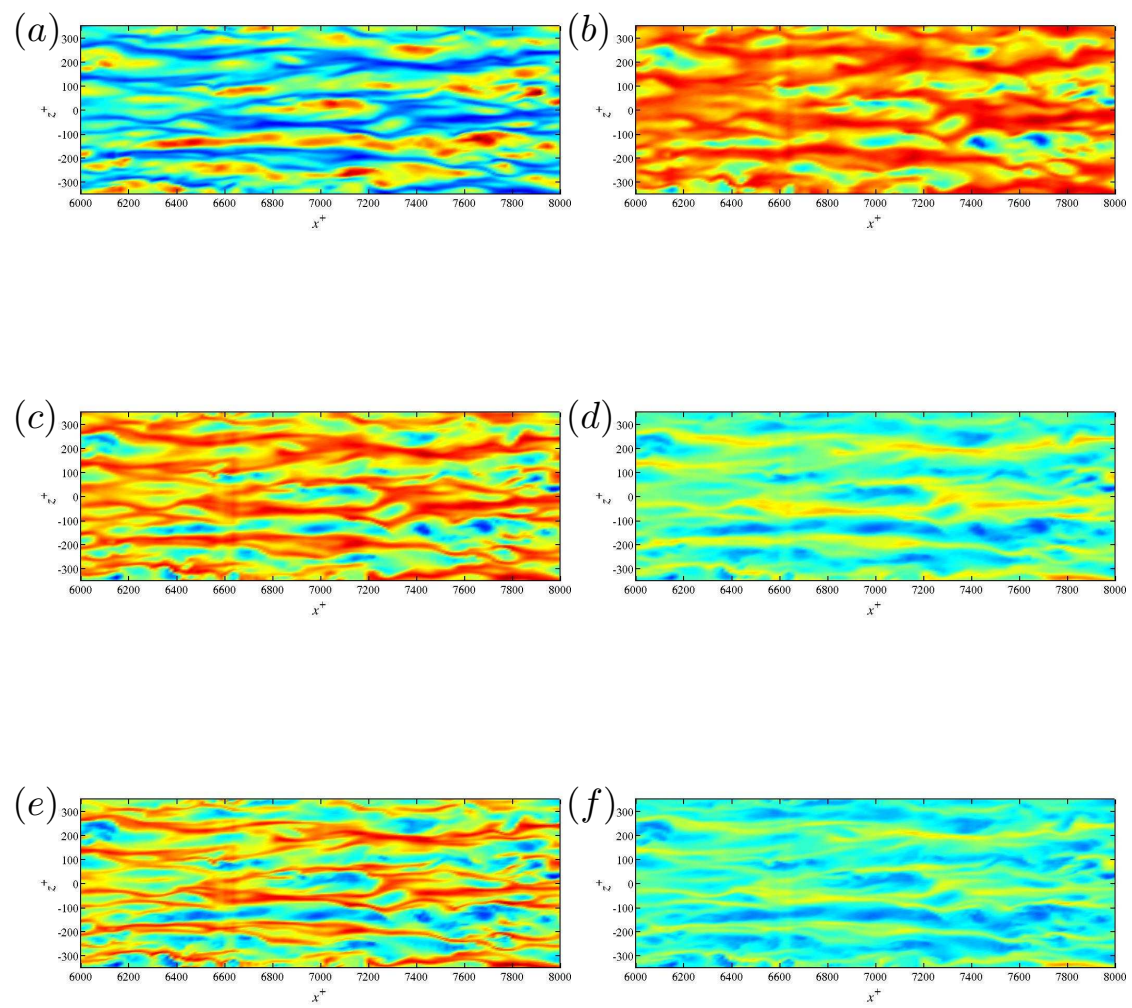

Figure 4.67: Instantaneous flow and scalar fields at $y^{+} \approx 7$. (a) $u^{\prime},(b) \theta_{1}^{\prime}$, (c) $\theta_{2}^{\prime},(d) \theta_{3}^{\prime},(e) \theta_{4}^{\prime},(f) \theta_{5}^{\prime}$.

plots shown here are only part of the computational domain and obtained at the same instant. The viscous units are defined by the friction velocity $u_{\tau}$ at about the center at the domain, i.e. $x=350$. Streaky structures are clearly observed in both the streamwise velocity and scalar fluctuation for the isothermal wall boundary condition at $\operatorname{Pr}=0.71$ and a strong similarity exists between them. The region of low and high scalar concentrations are elongated in the streamwise direction with a mean spanwise spacing similar to that of the streamwise velocity fluctuation. In the case of the isoflux wall boundary condition, the scalar streak spacing becomes larger than that for the isothermal case, see also Figure 4.55. It is also clear from Figure 4.67 that low-speed fluids are associated with low scalar concentration region and high-speed fluids with high scalar concentration region which is consistent with the experimental observation by Iritani et al. (1985). 


\section{Chapter 5}

\section{Conclusion and Outlook}

\subsection{Conclusion}

A direct numerical simulation (DNS) of a spatially developing turbulent boundary layer with passive scalars over a flat plate under zero pressure gradient (ZPG) is carried out. The Reynolds number based on the inlet displacement thickness $R e_{0}^{*}$ is 450 and Prandtl numbers are varying from 0.2 to 2 while two wall boundary conditions are employed. A spectral method, i.e. Fourier series in the horizontal directions and Chebychev expansion in the wall-normal direction, is used for the spatial derivatives in conjunction with Runge-Kutta and Crank-Nicolson schemes for time advancement. The computed velocity and scalar fields were compared with the existing experimental and numerical simulation data, in general, the agreement is very good.

The velocity profile agrees with the log-law very well while the mean scalar profiles are in good agreement with the empirical formula suggested by Kader (1981) and do not depend on the type of the wall boundary conditions employed. The mean scalar profiles for the isoflux boundary condition are slightly lower than those for the isothermal boundary condition in the logarithmic region, and the logarithmic region does not exist for $\operatorname{Pr}=0.2$ and becomes more distinguished as $\operatorname{Pr}$ increases. The skin friction coefficient and the Stanton numbers for different scalars all follow the solutions suggested by Kays and Crawford (1993). The turbulent Prandtl numbers with isothermal boundary condition seem to be independent of the molecular Prandtl number near the wall except the low Prandtl number case of $\operatorname{Pr}=0.2$ while they decrease to zero at the wall for the isoflux case.

With the increase of the Reynolds number, the increase of the wallnormal and spanwise velocity RMS fluctuation components in wall units is more enhanced than that of the streamwise component. For the scalar field, the RMS values of the scalar fluctuations retain non-zero values at the wall for isoflux boundary condition and become zero for isothermal boundary 
condition. Away from the wall, the RMS profiles of the scalars for both wall boundary conditions are almost identical. As $\operatorname{Pr}$ decreases, the scalar fluxes are also decreased with their peak positions moving away from the wall.

The Reynolds stress budgets reveal that several terms that are negligible away from the wall become more important close to the wall. The budget for the turbulence kinetic energy $k$ reveals that the velocity pressure-gradient term remains small relative to the dissipation and molecular diffusion terms near the wall. Away from the wall, the production and the dissipation terms are the dominant terms as estimated by Tennekes and Lumley (1972). The velocity pressure-gradient term acts as a dominant term in the wall-normal, spanwise and shear stress budgets. The same behaviour is also found for the scalar pressure-gradient term, but when the Prandtl number is low, it no longer acts as a dominant term for the scalar fluxes budgets. The similar distributions of the scalar flux and Reynolds stress budgets suggest that they might be generated by similar mechanisms and modelled in the same manner.

The structures of the velocity and scalar fields are also examined. It is found that the scalar of $\operatorname{Pr}=0.71$ is highly correlated with the streamwise velocity. The streaky structures found in the near wall region in the flow field are also observed in the scalar field with more or less the same nondimensional mean spacing which is almost independent of the molecular Prandtl numbers except the case for $\operatorname{Pr}=0.2$. The present spacings for both the velocity and scalar fields are slightly higher than the other simulation data and experimental results. This is attributed to the narrow box in the spanwise direction in the present DNS. By examining the spanwise two-point correlation coefficients of $u^{\prime}$ and $\theta^{\prime}$, we learn that the negative peaks become less prominent as the Reynolds number increases.

The probability density function (PDF) distributions of $u^{\prime}$ and $\theta^{\prime}$ can be approximated with Gaussian ones in the log-law region, but not in the other regions. Quadrant analyses and related joint probability density function (JPDF) distributions show that in general, both the velocity and scalar fluctuations are influenced strongly by the ejection and sweep types of motions, but less related with interaction-type motions. In the near wall region, the sweep motions play a dominant role in the production processes of Reynolds shear stress and wall-normal scalar flux and ejection motions predominate in the remaining region whereas the contributions from the interaction-type motions are negligible. However, for the isoflux boundary condition, the contributions of the wall-normal scalar flux from the interaction-type motions in the near wall region are not negligible unlike those for the isothermal wall boundary condition.

The instantaneous velocity and scalar fields near the wall show that a stronger coherence exists between the streamwise velocity $u$ and scalar of $\operatorname{Pr}=0.71$ for the isothermal wall than for the isoflux wall. The scalar streak spacings in case of the isoflux wall are larger than those in case of 
the isothermal wall. Again all these analyses of $u^{\prime}$ and $\theta^{\prime}$ underline the close similarity between the velocity and scalar fields close to the wall.

\subsection{Outlook}

Due to the computationally very expensive nature of the present calculation, not all the results are fully converged. Therefore, a natural extension of the present work is the continuation of the runs to obtain fully converged statistics, even for higher order ones. To make the code more effective, the parallelization in the streamwise direction in the code will be done in the future. It would be also interesting to make a longer and wider computational domain to reach a higher Reynolds number and to capture larger scale motions and structures in the outer region or to employ other different boundary conditions. By adopting a multi-grid approach or SGS modelling, we can examine scalar behaviours with a higher Pr. Some Large Eddy Simulations (LES), sub-grid models for both the flow and passive scalar could be done in the future based on the present DNS results. To look at the Reynolds analogy in the presence of free-stream turbulence (FST) with bypass transition is also worth doing. One could also try to implement active scalars to look at the instabilities, e.g. Rayleigh-Bénard problem or finger problem.

Here I would like to thank the computer centers. Computer time was provided by the Center for Parallel Computers (PDC) at the Royal Institute of Technology (KTH) and the National Supercomputer Center in Sweden (NSC) at Linköping University. 


\section{Acknowledgement}

First of all, I would like to thank my supervisor, Dr. Philipp Schlatter, who leads me into this challenging field of turbulence and whose knowledge in the numerical simulation really impressed me. Also I would like to thank him for his patience and carefulness in answering my questions and correcting the thesis. It is him who make the thesis work going in the right direction smoothly. (Danke sehr!) Another important person I would like to thank is Dr. Luca Brandt for his crucial suggestions of the thesis. He is always ready to help me whenever I got stuck. And his sense of humour brings me a lot of fun. (Grazie! Forza Italia!) Professor Dan Henningson is also highly acknowledged for creating this project for me and taking me as a student. (Tack så mycket!)

I would like to thanks all my teachers at departments of Mechanics and Solid Mechanics for very interesting and instructive lectures. I also want to thank the colleges in the group: Antonios Monokrousos, David Tempelmann, Espen Åkervik, Johan Olsson, Lars-Uve Schrader, Shervin Bagheri and coordinators from the master programme: Carina Ankarloo, Jean-Marc Battini and my friends in Sweden: Christer Wickman, Johan Hirvi, Rasmus Grip, Robert Petterson and others who are not mentioned here.

Special thanks to Mr. and Mrs. Von Bahr together with Helen and Peter for the inviting me to their home and making me feel warm in this frozen kingdom.

Then comes my "communist" friends: Jing Jing, Kang Yang, Kong Jie, Li Qiang, Li, Zhen, Men Shaodong, Shen Ruobing, Song Fei, Wang Shenshi, Zhang Tong, Zheng Ke, thank you for being my friends and for your support and encouragement, laughs and tears.

Thank you Lenngren, Lucidor and Mozart, base on your good and stable performance (even though some of you crashed for a few times), I can finish this thesis in time. And many thanks to MBC, SBS, KBS for making my life not so boring.

Finally, many thanks to mama and baba for bringing me up and devoting endless unconditional love and support to me. And I would also like to thank you, "my girl". However, it is a pity that I do not know who and where you are. Anyway, I wish you healthy and happy all the time. 


\section{Bibliography}

H. Abe, H. Kawamura, and Y. Matsuo. Direct numerical simulation of a fully developed turbulent channel flow with respect to the Reynolds number dependence. J. Fluids Engng, 382(123):382-393, 2001.

H. Abe, H. Kawamura, and Y. Matsuo. Surface heat-flux fluctuations in a turbulent channel flow up to $R e_{\tau}=1024$ with $\operatorname{Pr}=0.025$ and 0.71. Int. J. Heat Fluid Flow, 25(3):404-419, 2004.

P. H. Alfredsson and A. V. Johansson. On the detection of turbulencegenerating events. J. Fluid Mech., 139:325-345, 1984.

F. Anselmet, H. Djeridi, and L. Fulachier. Joint statistics of a passive scalar and its dissipation in turbulent flows. J. Fluid Mech., 280:173-197, 1994.

R. A. Antonia and H. Q. Danh. Structure of temperature fluctuations in a turbulent boundary layer. Phys. Fluids, 20(7):1050-1057, 1977.

R. A. Antonia and J. Kim. Turbulent Prandtl number in the near-wall region of a turbulent channel flow. Int. J. Heat Mass Transfer, 34(7):1905-1908, 1991.

R. A. Antonia and J. Kim. Low-Reynolds-number effects on near-wall turbulence. J. Fluid Mech., 276:61-80, 1994.

R. A. Antonia, L. V. Krishnamoorthy, and L. Fulachier. Correlation between the longitudinal velocity fluctuation and temperature fluctuation in the near-wall region of a turbulent boundary layer. Int. J. Heat Mass Transfer, 31(4):723-730, 1988.

G. I. Barenblatt. Scaling, Self-Similarity, and Intermediate Asymptotics. Cambridge University Press, Cambridge, U.K., 1996.

G. K. Batchelor. Small-scale variation of convected quantities like temperature in turbulent fluid: Part 1. General discussion and the case of small conductivity. J. Fluid Mech., 5:113-133, 1959.

A. Bejan. Convection Heat Transfer. John Wiley \& Sons, New York, U.S.A., $2^{\text {nd }}$ edition, 1995. 
A. Benaissa, J. Lemay, and F. Anselmet. Conditional correlation between a passive scalar and its dissipation in a turbulent boundary layer. Exps. Fluids, 26(6):488-496, 1999.

C. Canuto, M. Y. Hussaini, A. Quarteroni, and T. A. Zang. Spectral Methods in Fluid Dynamics. Springer, Berlin, Germany, 1988.

T. Cebeci and P. Bradshaw. Physical and Computational Aspects of Convective Heat Transfer. Springer, Berlin, Germany, 1984.

S. Y. Chung and H. J. Sung. Direct numerical simulation of turbulent concentric annular pipe flow. Part 2: Heat transfer. Int. J. Heat Fluid Flow, 24(3):399-411, 2003.

D. Coles. The law of the wake in the turbulent boundary layer. J. Fluid Mech., 1(2):191-226, 1956.

E. R. Corino and R. S. Brodkey. A visual investigation of the wall region in turbulent flow. J. Fluid Mech., 37:1-30, 1969.

S. Corrsin. Heat transfer in isotropic turbulence. J. Appl. Phys., 23(1): 113-118, 1952.

B. J. Daly and F. H. Harlow. Transport equations in turbulence. Phys. Fluids, 13(11):2634-2649, 1970.

F. Durst and K. N. Beronov. On the difficulties in resolving the viscous sublayer in wall-bounded turbulence. In Direct and Large-Eddy Simulation $V$, pages 117-124. Springer-Verlag, Berlin, 2003.

N. Gilbert and L. Kleiser. Turbulence model testing with the aid of direct numerical simulation results. In Eighth Symposium on Turbulent Shear Flows, pages 26-1-1-26-1-6, 1991.

A. K. Gupta, J. Laufer, and R. E. Kaplan. Spatial structure in the viscous sublayer. J. Fluid Mech., 50:493-512, 1971.

G. Hetsroni, T. A. Kowalewski, B. Hu, and A. Mosyak. Tracking of coherent thermal structures on a heated wall by means of infrared thermography. Exps. Fluids, 30(3):286-294, 2001.

J. O. Hinze. Turbulence. McGraw-Hill, New York, U.S.A., $2^{\text {nd }}$ edition, 1975.

M. Hishida and Y. Nagano. Structure of turbulent velocity and temperature fluctuations in fully developed pipe flow. ASME J. Heat Transfer, 101: 15-22, 1979.

P. H. Hoffmann and A. E. Perry. The development of turbulent thermal layers on flat plates. Int. J. Heat Mass Transfer, 22(1):39-46, 1979. 
Y. Iritani, N. Kasagi, and M. Hirata. Heat transfer mechanism and associated turbulence structure in a near wall region of a turbulent boundary layer. In L. J. S. Bradburry, F. Durst, B. E. Launder, F. W. Schmidt, and J. H. Whitelaw, editors, Turbulent Shear Flows 4, pages 223-234. Springer-Verlag, Berlin, 1985.

K. Iwamoto. Database of fully developed channel flow. Technical Report ILR-0201, Univ. of Tokyo, Tokyo, Japan, 2002.

R. G. Jacobs and P. A. Durbin. Bypass transition phenomena studies by computer simulation. Technical Report TF-77, Stanford University, U.S.A., 2000.

M. Jischa and H. B. Rieke. About the prediction of turbulent prandtl and schmidt numbers from modeled transport equations. Int. J. Heat Mass Transfer, 22(11):1547-1555, 1979.

B. A. Kader. Temperature and concentration profiles in fully turbulent boundary layers. Int. J. Heat Mass Transfer, 24(9):1541-1544, 1981.

B. A. Kader and A. M. Yaglom. Heat and mass transfer laws for fully turbulent wall flows. Int. J. Heat Mass Transfer, 15(12):2329-2351, 1972.

N. Kasagi and O. Iida. Progress in direct numerical simulation of turbulent heat transfer. In Proceedings of the 5th ASME/JSME Joint Thermal Engineering Conference, San Diego, U.S.A., 1999.

N. Kasagi, A. Kuroda, and M. Hirata. Numerical investigation of near-wall turbulent heat transfer taking into account the unsteady heat conduction in the solid wall. ASME J. Heat Transfer, 111(2):385-392, 1989.

N. Kasagi and Y. Ohtsubo. Direct numerical simulation of low Prandtl number thermal fluid in a turbulent channel flow. In F. Durst, editor, Turbulent Shear Flows 8, pages 97-119. Springer-Verlag, Berlin, 1993.

N. Kasagi, Y. Tomita, and A. Kuroda. Direct numerical simulation of passive scalar field in a turbulent channel flow. ASME J. Heat Transfer, 114:598606, 1992.

H. Kawamura, K. Ohsaka, H. Abe, and K. Yamamoto. DNS of turbulent heat transfer in channel flow with low to medium-high prandtl number fluid. Int. J. Heat Fluid Flow, 19(5):482-491, 1998.

W. M. Kays. Turbulent Prandtl number - Where are we?. ASME J. Heat Transfer, 116:284-295, 1994.

W. M. Kays and M. E. Crawford. Convective Heat and Mass Transfer. McGraw-Hill, New York, U.S.A., $3^{\text {rd }}$ edition, 1993. 
J. Kestin and P. D. Richardson. Heat transfer across turbulent, incompressible boundary layers. Int. J. Heat Mass Transfer, 6(2):147-189, 1963.

H. T. Kim, S. J. Kline, and W. C. Reynolds. The poduction of turbulence near a smooth wall in a turbulent boundary layer. J. Fluid Mech., 50: 133-160, 1971.

J. Kim. Investigation of heat and momentum transport in turbulent flows via numerical simulations. In International Symposia on Transport Phenomena: Transport Phenomena in Turbulent Flows: Theory, Experiment, and Numerical Simulation, pages 715-729. Hemisphere Publishing Corp., New York, 1988.

J. Kim. On the structure of pressure fluctuations in simulated turbulent channel flow. J. Fluid Mech., 205:421-451, 1989.

J. Kim and M. J. Lee. The structure of pressure fluctuations in turbulent shear flow. In F. Durst, B. E. Launder, W. C. Reynolds, F. W. Schmidt, and J. H. Whitelaw, editors, Turbulent Shear Flows 7, pages 87-99. Springer-Verlag, Berlin, 1991.

J. Kim and P. Moin. Transport of passive scalars in a turbulent channel flow. In J.-C. André, J. Cousteix, F. Durst, B. E. Launder, and F. W. Schmidt, editors, Turbulent Shear Flows 6, pages 85-96. Springer-Verlag, Berlin, 1989.

J. Kim, P. Moin, and R. Moser. Turbulence statistics in fully developed channel flow at low Reynolds number. J. Fluid Mech., 177:133-166, 1987.

S. J. Kline, W. C. Reynolds, F. A. Schraub, and P. W. Runstadler. The structure of turbulent boundary layers. J. Fluid Mech., 30:741-773, 1967.

J. Komminaho and M. Skote. Reynolds stress budgets in Couette and boundary layer flows. Flow, Turbulence Combust., 68(2):167-192, 2002.

H. Kong, H. Choi, and J. S. Lee. Direct numerical simulation of turbulent thermal boundary layers. Phys. Fluids, 12(10):2555-2568, 2000.

H. Kong, H. Choi, and J. S. Lee. Dissimilarity between the velocity and temperature fields in a perturbed turbulent thermal boundary layer. Phys. Fluids, 13(5):1466-1479, 2001.

H.-O. Kreiss and J. Oliger. Comparison of accurate methods for the integration of hyperbolic equations. Tellus, 24:199-215, 1972.

L. V. Krishnamoorthy and R. A. Antonia. Temperature-dissipation measurements in a turbulent boundary layer. J. Fluid Mech., 176:265-281, 1987. 
P. A. Libby. Introduction to Turbulence. Taylor \& Francis, New York, U.S.A, 1996.

D. M. Lu and G. Hetsroni. Direct numerical simulation of a turbulent open channel flow with passive heat transfer. Int. J. Heat Mass Transfer, 38 (17):3241-3251, 1995.

S. S. Lu and W. W. Willmarth. Measurements of the structure of the Reynolds stress in a turbulent boundary layer. J. Fluid Mech., 60:481511, 1973.

J. L. Lumley and G. R. Newman. The return to isotropy of homogeneous turbulence. J. Fluid Mech., 82:161-178, 1977.

A. Lundbladh, S. Berlin, M. Skote, C. Hildings, J. Choi, J. Kim, and D. S. Henningson. An efficient spectral method for simulation of incompressible flow over a flat plate. Technical Report TRITA-MEK 1999:11, Royal Institute of Technology, Stockholm, 1999.

S. L. Lyons and T. J. Hanratty. Direct numerical simulation of passive heat transfer in a turbulent channel flow. Int. J. Heat Mass Transfer, 34(4-5): 1149-1161, 1991.

N. N. Mansour, J. Kim, and P. Moin. Reynolds-stress and dissipation-rate budgets in a turbulent channel flow. J. Fluid Mech., 194:15-44, 1988.

P. Moin and J. Kim. Numerical investigation of turbulent channel flow. J. Fluid Mech., 118:341-377, 1982.

P. Moin and K. Mahesh. Direct numerical simulation: A tool in turbulence research. Annu. Rev. Fluid Mech., 30:539-578, 1998.

A. S. Monin and A. M. Yaglom. Statistical fluid mechanics: Mechanics of turbulence, volume 1. The MIT Press, Cambridge, U.S.A., 1971.

R. D. Moser, J. Kim, and N. N. Mansour. Direct numerical simulation of turbulent channel flow up to $R e_{\tau}=590$. Phys. Fluids, 11(4):943-945, 1999.

A. Mosyak, E. Pogrebnyak, and G. Hetsroni. Effect of constant heat flux boundary condition on wall temperature fluctuations. ASME J. Heat Transfer, 123(2):213-218, 2001.

Y. Na and T. J. Hanratty. Limiting behavior of turbulent scalar transport close to a wall. Int. J. Heat Mass Transfer, 43(10):1749-1758, 2000.

Y. Nagano and M. Tagawa. Statistical characteristics of wall turbulence with a passive scalar. J. Fluid Mech., 196:157-185, 1988. 
Y. Nagano and M. Tagawa. Coherent motions and heat transfer in a wall turbulent shear flow. J. Fluid Mech., 305:127-157, 1995.

J. Nordström, N. Nordin, and D. S. Henningson. The fringe region technique and the Fourier method used in the Direct Numerical Simulation of spatially evolving viscous flows. SIAM J. Sci. Comp., 20(4):1365-1393, 1999.

S. A. Orszag. Comparison of pseudospectral and spectral approximations. Stud. Appl. Math., 51:253-259, 1972.

J. M. Österlund. Experimental studies of zero pressure-gradient turbulent boundary layer flow. PhD thesis, Royal Institute of Technology, Stockholm, Sweden, 1999.

J. M. Österlund and A. V. Johansson. Turbulence statistics of zero pressuregradient turbulent boundary layers. Technical report, Royal Institute of Technology, Stockholm, Sweden, 1999.

J. M. Österlund, A. V. Johansson, H. M. Nagib, and M. H. Hites. A note on the overlap region in turbulent boundary layers. Phys. Fluids, 12(1): $1-4,2000$.

S. B. Pope. Turbulent Flows. Cambridge University Press, Cambridge, U.K., 2000.

L. P. Purtell, P. S. Klebanoff, and F. T. Buckley. Turbulent boundary layer at low Reynolds number. Phys. Fluids, 24(5):802-811, 1981.

M. M. Rai and P Moin. Direct numerical simulation of transition and turbulence in a spatially evolving boundary layer. J. Comp. Phys., 109(2): 169-192, 1993.

S. Rajagopalan and R. A. Antonia. Use of a quadrant analysis technique to identify coherent structures in a turbulent boundary layer. Phys. Fluids, 25(6):949-956, 1982.

P. E. Roach and D. H. Brierley. The influence of a turbulent freestream on zero pressure gradient transitional boundary layer development, part I: Test cases T3A and T3B. In Numerical Simulation of Unsteady Flows and Transition to Turbulence, ERCOFTAC, pages 319-347. Cambridge University Press, Cambridge, U.K., 1992.

S. K. Robinson. Coherent motions in the turbulent boundary layer. Ann. Rev. Fluid Mech., 23:601-639, 1991.

M. Rogers, P. Moin, and W. Reynolds. The structure and modeling of the hydrodynamic and passive scalar fields in homogeneous turbulent shear flow. Technical Report TF-25, Stanford University, U.S.A., 1986. 
A. Sahay and K. Sreenivasan. The wall-normal position in pipe and channel flow at which viscous and turbulent shear stresses are equal. Phys. Fluids, 11(10):3186-3188, 1999.

G. Schewe. On the structure and resolution of wall-pressure fluctuations associated with turbulent boundary-layer flow. J. Fluid Mech., 134:311$328,1983$.

P. Schlatter. Direct numerical simulation of laminar-turbulent transition in boundary layer subject to free-stream turbulence. Master's thesis, Institute of Fluid Dynamics, ETH, Zürich, Switzerland, 2001.

H. Schlichting. Boundary Layer Theory. McGraw-Hill, New York, U.S.A., $7^{\text {th }}$ edition, 1987.

K. E. Schoenherr. Resistance of flat surfaces moving through a fluid. Soc. Nav. Archit. Mar. Eng., 40:279, 1932.

A. Sirivat and Z. Warhaft. The effect of a passive cross-stream temperature gradient on the evolution of temperature variance and heat flux in grid turbulence. J. Fluid Mech., 128:323-346, 1983.

M. Skote. Studies of turbulent boundary layer flow through direct numerical simulation. PhD thesis, Royal Institute of Technology, Stockholm, Sweden, 2001.

C. R. Smith and S. P. Metzler. The characteristics of low-speed streaks in the near-wall region of a turbulent boundary layer. J. Fluid Mech., 129: 27-54, 1983.

A. J. Smits, N. Matheson, and P. N. Jourbert. Low-Reynolds-number turbulent boundary layers in zero and favorable pressure gradients. J. Ship Res., 27:147, 1983.

P. R. Spalart. Direct simulation of a turbulent boundary layer up to $R e_{\theta}=$ 1410. J. Fluid Mech., 187:61-98, 1988.

S. Tavoularis and S. Corrsin. Experiments in nearly homogenous turbulent shear flow with a uniform mean temperature gradient. J. Fluid Mech., 104:311-367, 1981.

H. Tennekes and J. L. Lumley. A First Course in Turbulence. The MIT Press, Cambridge, U.S.A., 1972.

I. Tiselj, R. Bergant, B. Mavko, I. Bajsić, and G. Hetsroni. DNS of turbulent heat transfer in channel flow with heat conduction in the solid wall. $A S M E$ J. Heat Transfer, 123:849-857, 2001a. 
I. Tiselj, E. Pogrebnyak, C. F. Li, A. Mosyak, and G. Hetsroni. Effect of wall boundary condition on scalar transfer in a fully developed turbulent flume. Phys. Fluids, 13(4):1028-1039, 2001b.

J. M. Wallace, H. Eckelmann, and R. S. Brodkey. The wall region in turbulent shear flow. J. Fluid Mech., 54:39-48, 1972.

Z. Warhaft and J. L. Lumley. An experimental study of the decay of temperature fluctuations in grid-generated turbulence. J. Fluid Mech., 88: 659-685, 1978.

C. E. Wark and H. M. Nagib. Experimental investigation of coherent structures in turbulent boundary layers. J. Fluid Mech., 230:183-208, 1991.

K. J. A. Westin, A. V. Boiko, B. G. B. Klingmann, V. V. Kozlov, and P. H. Alfredsson. Experiments in a boundary layer subjected to free stream turbulence. Part 1. Boundary layer structure and receptivity. J. Fluid Mech., 281:193-218, 1994.

F. M. White. Viscous fluid flow. McGraw-Hill, New York, U.S.A., $3^{\text {rd }}$ edition, 2006.

P. Wikström. Measurements, direct numerical simulation and modeling of passive scalar transport in turbulent flows. PhD thesis, Royal Institute of Technology, Stockholm, Sweden, 1998.

W. W. Willmarth and S. S. Lu. Structures of the Reynolds stress near the wall. J. Fluid Mech., 55:65-92, 1972.

Z. Zarić. Wall turbulence structure and convection heat transfer. Int. J. Heat Mass Transfer, 18(6):831-842, 1975.

Y. Zhu and R. A. Antonia. Temperature dissipation measurements in a fully developed turbulent channel flow. Exps. Fluids, 15(3):191-199, 1993. 